\title{
745.
}

\section{ON THE SCHWARZIAN DERIVATIVE, AND THE POLYHEDRAL FUNCTIONS.}

[From the Transactions of the Cambridge Philosophical Society, vol. xIII. Part I. (1881), pp. 5-68. Read March 8, 1880.]

THE quotient $s$ of any two solutions of a linear partial differential equation of the second order, $\frac{d^{2} y}{d x^{2}}+p \frac{d y}{d x}+q y=0$, is determined by a differential equation of the third order

$$
\frac{\frac{d^{3} s}{d x^{3}}}{\frac{d s}{d x}}-\frac{3}{2}\left(\frac{\frac{d^{2} s}{d x^{2}}}{\frac{d s}{d x}}\right)^{2}=-\frac{1}{2}\left(p^{2}+2 \frac{d p}{d x}-4 q\right)
$$

where the function on the left-hand is what I call the-Schwarzian Derivative; or say this derivative is

$$
\{s, x\},=\frac{s^{\prime \prime \prime}}{s^{\prime}}-\frac{3}{2}\left(\frac{s^{\prime \prime}}{s^{\prime}}\right)^{2},
$$

where the accents denote differentiations in regard to the second variable $x$ of the symbol.

Writing in general $(\mathrm{a}, \mathrm{b}, \mathrm{c} \therefore \chi X, Y, Z)^{2}$ to denote a quadric function

$$
\left(a, b, c, \frac{1}{2}(a-b-c), \quad \frac{1}{2}(-a+b-c), \quad \frac{1}{2}(-a-b+c) \gamma X, Y, Z\right)^{2},
$$

then, if the equation of the second order be that of the hypergeometric series, generalised by a homographic transformation upon the variable $x$, the resulting differential equation of the third order is of the form

$$
\{s, x\}=(\mathrm{a}, \mathrm{b}, \mathrm{c} \therefore)\left(\frac{1}{x-a}, \frac{1}{x-b}, \frac{1}{x-c}\right)^{2} ;
$$


and, presenting themselves in connexion with the algebraically integrable cases of this equation, we have rational and integral functions of $s$, derived from the polygon, the double pyramid, and the five regular solids. They are called Polyhedral Functions.

The Schwarzian Derivative occurs implicitly in Jacobi's differential equation of the third order for the modulus in the transformation of an elliptic function (Fund. Nova, 1829 , p. 79, [Ges. Werke, t. I., p. 133]) and in Kummer's fundamental equation for the transformation of a hypergeometric series (Kummer, 1836: see list of Memoirs): but it was first explicitly considered and brought into notice in the two Memoirs of Schwarz*, 1869 and 1873. The latter of these, relating to the algebraic integration of the differential equation for the hypergeometric series, is the fundamental Memoir upon the subject, but the theory is in some material points completed in the Memoirs by Klein and Brioschi.

The following list of Memoirs, relating as well to the Polyhedral Functions as to the Schwarzian Derivative, is arranged nearly in chronological order.

Kummer, Ueber die hypergeometrische Reihe $1+\frac{\alpha \cdot \beta}{1 \cdot \gamma} x+\ldots$ Crelle, t. xv. (1836), pp. $39-83$ and $127-172$.

Schwarz, Ueber einige Abbildungsaufgaben. Crelle-Borchardt, t. Lxx. (1869), pp. 105-120.

Ueber diejenigen Fälle in welchen die Gaussische hypergeometrische Reihe eine algebraische Function ihres vierten Elementes darstellt. Do. t. Lxxv. (1873), pp. 292-335.

Cayley, Notes on Polyhedra. Quart. Math. Jour. t. vir. (1866), pp. 304-316; [375]. On the Regular Solids. Do. t. xv. (1878), pp. 127-131; [679].

Fuchs, Ueber diejenigen Differentialgleichungen zweiter Ordnung welche algebraische Integralen besitzen, und eine Anwendung der Invariantentheorie. Crelle-Borchardt, t. LXXXI. (1875), pp. 97-142.

Klein, Ueber binäre Formen mit linearen Transformationen in sich selbst. Math. Ann. t. Ix. (1875), pp. 183-209.

Brioschi, Extrait d'une lettre à M. Klein. Math. Ann. t. XI. (1877), pp. 111-114.

Klein, Ueber lineare Differentialgleichungen. Math. Ann. t. XI. (1877), pp. 115-118.

Brioschi, La théorie des formes dans l'intégration des équations différentielles linéaires du second ordre. Math. Ann. t. XI. (1877), pp. 401-411.

Gordan, Ueber endliche Gruppen linearer Transformationen einer Veränderlichen. Math. Ann. t. xII. (1877), pp. 23-46.

Binäre Formen mit verschwindenden Covarianten. Math. Ann. t. XII. (1877), pp. $147-166$.

[* Schwarz, Ges. Werke, t. II., p. 351, remarks that the Derivative occurs implicitly in a memoir by Lagrange, "Sur la construction des cartes géographiques," (1779), EEuvres, t. Iv., p. 651.] 
Klein, Ueber lineare Differentialgleichungen. Math. Ann. t. XII. (1877), pp. 167-179.

Weitere Untersuchungen über das Icosaeder. Math. Ann. t. xII. (1877), pp. $503-560$.

Cayley, On the Correspondence of Homographies and Rotations. Math. Ann. t. xv. (1879), pp. 238-240; [660].

On the finite Groups of linear transformations of a Variable. Math. Ann. t. XVI. (1880), pp. 260-263, and pp. 439-440; [752].

I propose in the present Memoir to consider the whole theory: and, in particular, to give some additional developments in regard to the Polyhedral Functions.

I remark that Schwarz starts with the foregoing differential equation of the third order

$$
\{s, x\}=(\mathrm{a}, \mathrm{b}, \mathrm{c} \therefore)\left(\frac{1}{x-a}, \frac{1}{x-b}, \frac{1}{x-c}\right)^{2},
$$

and he shows (by very refined reasoning founded on the theory of conformable figures, which will be in part reproduced) that this equation is, in fact, algebraically integrable for 16 different sets of values of the coefficients a, b, c. It may I think be taken to be part of his theory, although not very clearly brought out by him, that these integrals are some of them of the form, $x=$ rational function of $s$; others of the form, rational function of $x=$ rational function of $s$; the rational functions of $s$ being in fact the same in the last as in the first set of solutions: they are quotients of Polyhedral functions.

But as regards the second set of cases, the solution of these, introducing for convenience a new variable $z$ in place of $s$, may be made to depend upon the solution in the form, $x=$ rational function of $z$, of an equation of a somewhat similar form, but involving two quadric functions of $x$ and $z$ respectively, viz. the equation

$$
\{x, z\}+\left(\frac{d x}{d z}\right)^{2}(a, b, c \quad \therefore)\left(\frac{1}{x-a}, \frac{1}{x-b}, \frac{1}{x-c}\right)^{2}=\left(a_{1}, b_{1}, c_{1} \therefore\right)\left(\frac{1}{z-a_{1}}, \frac{1}{z-b_{1}}, \frac{1}{z-c_{1}}\right)^{2} ;
$$

and we have the theorem that the solution of this equation depends upon the determination of $P, Q, R$ rational and integral functions of $z$, containing each of them multiple factors, which are such that $P+Q+R=0$. Using accents to denote differentiation in regard to $z$, this implies $P^{\prime}+Q^{\prime}+R^{\prime}=0$, and consequently

$$
Q R^{\prime}-Q^{\prime} R=R P^{\prime}-R^{\prime} P=P Q^{\prime}-P^{\prime} Q .
$$

Further, they are such that the equal functions $Q R^{\prime}-Q^{\prime} R, R P^{\prime}-R^{\prime} P, P Q^{\prime}-P^{\prime} Q$ contain only factors which are factors of $P, Q$ or $R$.

In fact, writing $f, g, h=b-c, c-a, a-b$, the required relation between $x, z$ is then expressed in the symmetrical form $f(x-a): g(x-b): h(x-c)=P: Q: R$. 
The last-mentioned differential equation is considered by Klein and Brioschi: the solutions in 13 cases, or such of them as had not been given by Schwarz, were obtained by Brioschi: and those of the remaining 3 cases, subject to a correction in one of them, were afterwards obtained by Klein.

The first part of the present Memoir relates, say to the foregoing equation

$$
\{s, x\}=(\mathrm{a}, \mathrm{b}, \mathrm{c} \therefore)\left(\frac{1}{x-a}, \frac{1}{x-b}, \frac{1}{x-c}\right)^{2},
$$

although the other form in $\{x, z\}$ may equally well be regarded as the fundamental form.

We consider in the theory:

A. The Derivative $\{s, x\}$, meaning as above explained.

B. Quadric functions of any three or more inverts $\frac{1}{x-l}$.

C. Rational and integral functions $P, Q, R$ having a sum $=0$, and which are such that $Q R^{\prime}-Q^{\prime} R,=R P^{\prime}-R^{\prime} P,=P Q^{\prime}-P^{\prime} Q$, contains only the factors of $P, Q, R$.

D. The differential equation of the third order.

E. The Schwarzian theory in regard to conformable figures and the corresponding values of the imaginary variables $s$ and $x$.

F. Connexion with the differential equation for the hypergeometric series.

The second part of the Memoir relates to the Polyhedral Functions.

The paragraphs of the whole Memoir are numbered consecutively.

\section{PART I.}

The Derivative $\{s, x\}$. Art. Nos. 1 to 7 .

1. If $p=\frac{s^{\prime \prime}}{s^{\prime}}=\frac{d}{d x}\left(\log \frac{d s}{d x}\right)$, then $\{s, x\}=\frac{d p}{d x}-\frac{1}{2} p^{2}$.

2. The derivative $\{s, x\}$ may be transformed in regard to either or both of the variables.

Suppose, first, that $s$ is a function of the new variable $S$, (hence also $S$ is a function of $x$ ): using subscript numbers to denote differentiations in regard to $S$, and the accents as before for differentiations in regard to $x$, we have

$$
s^{\prime}=S^{\prime} s_{1},
$$

whence, differentiating the logarithms,

$$
\frac{s^{\prime \prime}}{s^{\prime}}=S^{\prime} \frac{s_{2}}{s_{1}}+\frac{S^{\prime \prime}}{S^{\prime}}
$$


Again differentiating, we have

$$
\frac{s^{\prime \prime \prime}}{s^{\prime}}-\left(\frac{s^{\prime \prime}}{s^{\prime}}\right)^{2}=S^{\prime 2}\left[\frac{s_{3}}{s_{1}}-\left(\frac{s_{2}}{s_{1}}\right)^{2}\right]+S^{\prime \prime} \frac{s_{2}}{s_{1}}+\frac{S^{\prime \prime \prime}}{S^{\prime}}-\left(\frac{S^{\prime \prime}}{S^{\prime \prime}}\right)^{2} .
$$

But

and consequently

$$
-\frac{1}{2}\left(\frac{s^{\prime \prime}}{s^{\prime}}\right)^{2}=S^{\prime 2}\left[-\frac{1}{2}\left(\frac{s_{2}}{s_{1}}\right)^{2}\right]-S^{\prime \prime} \frac{s_{2}}{s_{1}} \quad-\frac{1}{2}\left(\frac{S^{\prime \prime \prime}}{S^{\prime \prime}}\right)^{2}
$$

that is,

$$
\frac{s^{\prime \prime \prime}}{s^{\prime}}-\frac{3}{2}\left(\frac{s^{\prime \prime}}{s^{\prime}}\right)^{2}=S^{\prime 2}\left[\frac{s_{3}}{s_{1}}-\frac{3}{2}\left(\frac{s_{2}}{s_{1}}\right)^{2}\right] \quad+\frac{S^{\prime \prime \prime}}{S^{\prime \prime}}-\frac{3}{2}\left(\frac{S^{\prime \prime \prime}}{S^{\prime \prime}}\right)^{2}
$$

the required formula.

$$
\{s, x\}=\left(\frac{d S}{d x}\right)^{2}\{s, S\}+\{S, x\}
$$

In a very similar manner, taking $x$ a function of $X$, it is shown that

$$
\{s, x\}=\left(\frac{d X}{d x}\right)^{2}(\{s, X\}-\{x, X\}) .
$$

3. If in this formula we write $S$ for $s$, and substitute the resulting value of $\{S, x\}$ in the former formula, we have

$$
\{s, x\}=\left(\frac{d S}{d x}\right)^{2}\{s, S\}-\left(\frac{d X}{d x}\right)^{2}\{x, X\}+\left(\frac{d X}{d x}\right)^{2}\{S, X\},
$$

which is the formula for the change of both variables. It, in fact, includes the other two: viz. writing $X=x$, or $S=s$, and observing that $\{s, s\}=0=\{x, x\}$, we have the other two formulæ.

4. By putting in the first formula $X=s$, we obtain

$$
\{s, x\}=-\left(\frac{d s}{d x}\right)^{2}\{x, s\}
$$

a formula for the interchange of the variables.

5. Writing $S=\frac{a s+b}{c s+d}$, and using for a moment the accents to denote differentiation in regard to $s$, we have

and thence

$$
S^{\prime}=\frac{a d-b c}{(c s+d)^{2}}, \quad \frac{S^{\prime \prime}}{S^{\prime}}=\frac{-2 c}{c s+d}
$$

$$
\begin{gathered}
\frac{S^{\prime \prime \prime}}{S^{\prime \prime}}-\left(\frac{S^{\prime \prime}}{S^{\prime \prime}}\right)^{2}=\frac{2 c^{2}}{(c s+d)^{2}} \\
-\frac{1}{2}\left(\frac{S^{\prime \prime}}{S^{\prime \prime}}\right)^{2}=\frac{-2 c^{2}}{(c s+d)^{2}} .
\end{gathered}
$$

Consequently $\{S, s\}=0$, whence also $\{s, S\}=0$. 
Hence in the first formula $\{S, x\}=\{s, x\}$, that is,

$$
\left\{\frac{a s+b}{c s+d}, x\right\}=\{s, x\} \text {; }
$$

viz. we may, in the derivative $\{s, x\}$, write for $s$ any homographic function $(a s+b) \div(c s+d)$ of $s$.

6. Again, if $X=\frac{\alpha x+\beta}{\gamma x+\delta}$, then from the second formula

that is,

$$
\{s, x\}=\frac{(\alpha \delta-\beta \gamma)^{2}}{(\gamma x+\delta)^{4}}\{s, X\}
$$

$$
\left\{s, \frac{\alpha x+\beta}{\gamma x+\delta}\right\}=\frac{(\gamma x+\delta)^{4}}{(\alpha \delta-\beta \gamma)^{2}}\{s, x\}
$$

and here, changing $s$ into $(a s+b) \div(c s+d)$, we have finally

$$
\left\{\frac{a s+b}{c s+d}, \frac{a x+\beta}{\gamma x+\delta}\right\}=\frac{(\gamma x+\delta)^{4}}{(\alpha \delta-\beta \gamma)^{2}}\{s, x\},
$$

which is the formula for the homographic transformation of the two variables $s, x$.

7. Let $s$ be a given function of $x$, the equation $\{S, x\}=\{s, x\}$ is a differential equation of the third order in $S$, and by what precedes, its general integral is $S=\frac{a s+b}{c s+d}$.

The direct process is as follows: we have a first integral $\frac{S^{\prime \prime}}{S^{\prime \prime}}=\frac{s^{\prime \prime}}{s^{\prime}}-\frac{2 c s^{\prime}}{c s+d}$; a second integral $\log S^{\prime \prime}=\log s^{\prime}-2 \log (c s+d)+$ const., that is, $S^{\prime \prime}=\frac{c A s^{\prime}}{(c s+d)^{2}}$; and thence a final integral $S=B-\frac{A}{c s+d}$, which is equivalent to the foregoing value of $S$.

The Quadric Function of three or more Inverts. Art. Nos. 8 to 15.

8. We consider a quadric function of any number of inverts $\frac{1}{x-\alpha}, \frac{1}{x-\beta}, \ldots$, all of them different: it is assumed that the constant term is $=0$, and also that the sum of the coefficients of the linear terms is $=0$. We have therefore square terms $\frac{a}{(x-\alpha)^{2}}$, product terms $\frac{h}{x-\alpha \cdot x-\beta}$, and linear terms $\frac{A}{x-\alpha}$, where the sum of the coefficients $A$ is $=0$. Any product term $\frac{h}{x-\alpha \cdot x-\beta}$ is expressible in the form of a difference $\frac{h}{\alpha-\beta} \frac{1}{x-\alpha}-\frac{h}{\alpha-\beta} \frac{1}{x-\beta}$ of two linear terms, and (the coefficients of these

C. XI. 
being equal), after it is thus expressed, the sum of the coefficients of the linear terms is still $=0$. The function is thus always expressible in the form

$$
\frac{a}{(x-\alpha)^{2}}+\frac{b}{(x-\beta)^{2}}+\ldots+\frac{A}{x-\alpha}+\frac{B}{x-\beta}+\ldots
$$

where the sum $A+B+\ldots$ is $=0$ : this may be called the reduced form.

9. Observe that any particular invert $\frac{1}{x-\alpha}$ may disappear altogether from the reduced form: this will be the case if $a=0$, that is, if the original form contains no term in $\frac{1}{(x-\alpha)^{2}}$, and if also $A=0$. An invert thus disappearing from the reduced form is said to be non-essential: and the inverts which do not disappear are said to be essential. The original form contains in appearance the non-essential inverts, but it is really a quadric function of the essential inverts only.

10. Imagine the original function expressed as a rational fraction, the denominator being the product $(x-\alpha)^{2}(x-\beta)^{2}(x-\gamma)^{2} \ldots$ of the squared factors corresponding to all the inverts (non-essential as well as essential): the numerator will be in general of a degree less by 2 than that of the denominator, but the coefficients of any one or more of the higher powers of $x$ may vanish, and the numerator will then be of a lower degree. But this numerator will for any non-essential invert $\frac{1}{x-\gamma}$ contain the factor $(x-\gamma)^{2}$, or, dividing the numerator and denominator each by this factor, the difference of the degrees of the numerator and denominator will remain unaltered; that is, the difference will have the same value whether we do or do not attend to the non-essential inverts; or say it will have the same value for the original form and for the reduced form.

11. It is to be remarked that the linear terms $\frac{A}{x-\alpha}+\frac{B}{x-\beta}+\frac{C}{x-\gamma}+\ldots$, where $A+B+C+\ldots=0$, can be (and that in a variety of ways) expressed as a sum of differences $\frac{1}{x-\alpha}-\frac{1}{x-\beta}$, that is, as a sum of product-terms $\frac{1}{x-\alpha \cdot x-\beta}$. Hence the quadric function can be (and that in a variety of ways) expressed as a homogeneous function $\left.(a, \ldots) \frac{1}{x-\alpha}, \frac{1}{x-\beta}, \ldots\right)^{2}$; we must have in the form all the essential inverts, and we need have these only. Supposing that this is so, and that the number of the essential inverts is $=n$, then the number of constants is $=\frac{1}{2} n(n+1)$, whereas the number of constants in the reduced form is only $=2 n-1$ : hence the coefficients are not determinate; or, what is the same thing, we may have different quadric functions having each of them the same reduced function; these quadric functions, as having the same reduced function, can only differ by multiples of the evanescent expressions

$$
\frac{\beta-\gamma}{x-\beta \cdot x-\gamma}+\frac{\gamma-\alpha}{x-\gamma \cdot x-\alpha}+\frac{\alpha-\beta}{x-\alpha \cdot x-\beta}, \& c .
$$


In particular, if the number of essential inverts is $=3$, then the quadric function is of the form

$$
\left(\mathrm{a}, \mathrm{b}, \mathrm{c}, \mathrm{f}, \mathrm{g}, \mathrm{h} \gamma \frac{1}{x-\alpha}, \frac{1}{x-\beta}, \frac{1}{x-\gamma}\right)^{2}
$$

which contains one superfluous constant, and equivalent functions differ only by a multiple of

$$
\frac{\beta-\gamma}{x-\beta \cdot x-\gamma}+\frac{\gamma-\alpha}{x-\gamma \cdot x-\alpha}+\frac{\alpha-\beta}{x-\alpha \cdot x-\beta} .
$$

12. A quadric function such that the degree of the numerator is less by 4 than that of the denominator is said to be "curtate."

The conditions, in order that the function

$$
\left(\mathrm{a}, \mathrm{b}, \mathrm{c}, \mathrm{f}, \mathrm{g}, \mathrm{h} \gamma\left(\frac{1}{x-\alpha}, \frac{1}{x-\beta}, \frac{1}{x-\gamma}\right)^{2}\right.
$$

may be curtate, are easily found to be

$$
\begin{gathered}
a+b+c+2 f+2 g+2 h=0 \\
\alpha(a+h+g)+\beta(h+b+f)+\gamma(g+f+c)=0
\end{gathered}
$$

and by reason of the superfluous constant we are at liberty to assume a third condition: the three conditions may be taken to be $a+h+g, h+b+f, g+f+c$ each $=0$; and this being so the values of $f, g, h$ are $=\frac{1}{2}(a-b-c), \frac{1}{2}(-a+b-c), \frac{1}{2}(-a-b+c)$ respectively. Hence the form is

$$
\left(a, b, c, \frac{1}{2}(a-b-c), \frac{1}{2}(-a+b-c), \frac{1}{2}(-a-b+c)\left(\frac{1}{x-\alpha}, \frac{1}{x-\beta}, \frac{1}{x-\gamma}\right)^{2},\right.
$$

which, as already mentioned, we denote by

$$
\left(a, b, c \therefore \gamma \frac{1}{x-\alpha}, \frac{1}{x-\beta}, \frac{1}{x-\gamma}\right)^{2} \text {. }
$$

We have thus the theorem that a curtate function of any number of inverts, but with only the three essential inverts

$$
\frac{1}{x-\alpha}, \frac{1}{x-\beta}, \frac{1}{x-\gamma}
$$

is always expressible in the foregoing form

$$
\left(\mathrm{a}, \mathrm{b}, \mathrm{c} \therefore \gamma\left(\frac{1}{x-\alpha}, \frac{1}{x-\beta}, \frac{1}{x-\gamma}\right)^{2}\right. \text {. }
$$

13. It may be remarked that the function ( $\mathrm{a}, \mathrm{b}, \mathrm{c} \therefore X X, Y, Z)^{2}$ is a function of the differences of the variables $X, Y, Z$; and similarly, in the case of four variables, a function (a, b, c, d, f, g, h, l, m, n $\gamma X, Y, Z, W)^{2}$, for which

$$
a+h+g+l, \quad h+b+f+m, \quad g+f+c+n, \quad l+m+n+d,
$$


are each $=0$, is a function of the differences of the variables $X, Y, Z, W:$ and so in general. Any such function is said to be "diaphoric": and it is easy to see that, taking for the variables any inverts whatever, a diaphoric function is always curtate.

14. The function

$$
\begin{aligned}
& \left\{-\frac{a}{(x-\alpha)^{2}}-\frac{b}{(x-\beta)^{2}}-\frac{c}{(x-\gamma)^{2}}-\ldots\right\} \\
& -\frac{1}{2}\left\{\frac{a}{x-\alpha}+\frac{b}{x-\beta}+\frac{c}{x-\gamma}+\ldots\right\}^{2},
\end{aligned}
$$

where the coefficients $a, b, c, \ldots$ satisfy the relation $a+b+c+\ldots=-2$, is diaphoric, and therefore curtate. In fact, forming the sum, coeff. $\frac{1}{(x-\alpha)^{2}}+\frac{1}{2}$ coeff. $\frac{1}{x-\alpha \cdot x-\beta}+\ldots$, this is $-a-\frac{1}{2} a^{2}-\frac{1}{2} a b-\frac{1}{2} a c-\ldots,=-\frac{1}{2} a(2+a+b+c+\ldots)$, which is $=0$; and similarly the other conditions are satisfied.

15. The function

$$
\left(\mathrm{a}, \mathrm{b}, \mathrm{c} \therefore \gamma \frac{\mathrm{a}}{x-\alpha}+\frac{a_{1}}{x-\alpha_{1}}+\ldots, \quad \frac{b}{x-\beta}+\frac{b_{1}}{x-\beta_{1}}+\ldots, \frac{c}{x-\gamma}+\frac{c_{1}}{x-\gamma_{1}}+\ldots\right)^{2},
$$

regarded as a function of the inverts

$$
\frac{1}{x-\alpha}, \frac{1}{x-\alpha_{1}}, \ldots, \frac{1}{x-\beta}, \ldots
$$

where

$$
a+a_{1}+\ldots=b+b_{1}+\ldots=c+c_{1}+\ldots,=k \text { suppose }
$$

is diaphoric, and therefore curtate. In fact, the condition in regard to $\frac{1}{x-\alpha}$ is

$$
a\left(a^{2}+a a_{1}+a a_{2}+\ldots\right)+\frac{1}{2}(-a+b-c)\left(a b+a b_{1}+\ldots\right)+\frac{1}{2}(-a-b+c)\left(a c+a c_{1}+\ldots\right)=0 ;
$$

that is,

$$
a k\left\{a+\frac{1}{2}(-a+b-c)+\frac{1}{2}(-a-b+c)\right\}=0,
$$

which is satisfied. And similarly the other conditions are satisfied.

The functions $P, Q, R$. Art. Nos. 16 to 20 .

16. We consider $P, Q, R$, rational and integral functions of $z$, such that $P+Q+R=0$ : hence, using the accent to denote differentiation in regard to $z$, we have also $P^{\prime}+Q^{\prime}+R^{\prime}=0$; and therefore $Q R^{\prime}-Q^{\prime} R=R P^{\prime}-R^{\prime} P=P Q^{\prime}-P^{\prime} Q,=\Theta$ suppose : and we require to find $P, Q, R$ such that the function $\Theta$ contains only the factors of $P, Q, R$.

17. It is to be observed that, effecting upon a solution $P, Q, R$ any linear substitution $(\alpha z+\beta) \div(\gamma z+\delta)$, and omitting the common denominator, we have a solution; but this is regarded as identical with the original solution. The three functions, if 
not originally of the same order, can thus be made to be of the same order; or by taking account of the root $z=\infty$, we may in the original case regard them as being of the same order, and it is convenient so to regard them: say they are taken to be of the same order $\delta$. And there is clearly no loss of generality in taking the three functions to be prime to each other; for any common factor of two of them would divide the third, and might therefore be struck out.

18. We may therefore write

$$
P=F \Pi(z-l)^{p}, \quad Q=G \Pi(z-m)^{q}, \quad R=H \Pi(z-n)^{r},
$$

where $(z-l)^{p}$ is taken to denote the distinct simple or multiple factors of $P$, and the like as regards $Q$ and $R$; the factors $z-l, z-m, z-n$ are thus all of them different. And we have $\delta=\Sigma p,=\Sigma q,=\Sigma r$.

19. It is at once seen that $\Theta$ is of the degree $2 \delta-2$, and moreover that it contains the factors $\Pi(z-l)^{p-1}, \Pi(z-m)^{q-1}, \Pi(z-n)^{r-1}$; hence it contains the factor

$$
\Pi(z-l)^{p-1}(z-m)^{q-1}(z-n)^{r-1} .
$$

Suppose the number of distinct indices $p$ is $=\sigma_{1}$, that of distinct indices $q$ is $\sigma_{2}$, and that of distinct indices $r$ is $\sigma_{3}$; then the degree of the factor is $=3 \delta-\sigma_{1}-\sigma_{2}-\sigma_{3}$; and if this be $=2 \delta-2$, then $\Theta$ can have no other variable factor: viz. if the numbers $\sigma_{1}, \sigma_{2}, \sigma_{3}$ of the distinct indices $p, q, r$ respectively are such that $\sigma_{1}+\sigma_{2}+\sigma_{3}=\delta+2$, a relation which is henceforth taken to be satisfied, then we have

$$
\Theta=K \Pi(z-l)^{p-1}(z-m)^{q-1}(z-n)^{r-1} .
$$

As already in effect remarked, the conclusion extends to the case where $P, Q, R$ are not of the same degree; the equation $P+Q+R=0$ here implies that two functions, say $P, Q$, are of the same degree, and the third function $R$ of an inferior degree; but, this being so, we have only to regard $R$ as containing the factor $\left(1-\frac{z}{\infty}\right)^{t}$ of the degree $t$ proper for raising its degree up to that of $P$ or $Q$.

20. Solutions are given in the following $P Q R$-Table: in which, where required, the proper factor $\left(1-\frac{z}{\infty}\right)^{t}$ has been added; the first column headed Ref. No. (Reference Number) will be explained further on. The Annex to the same Table will also be explained. 


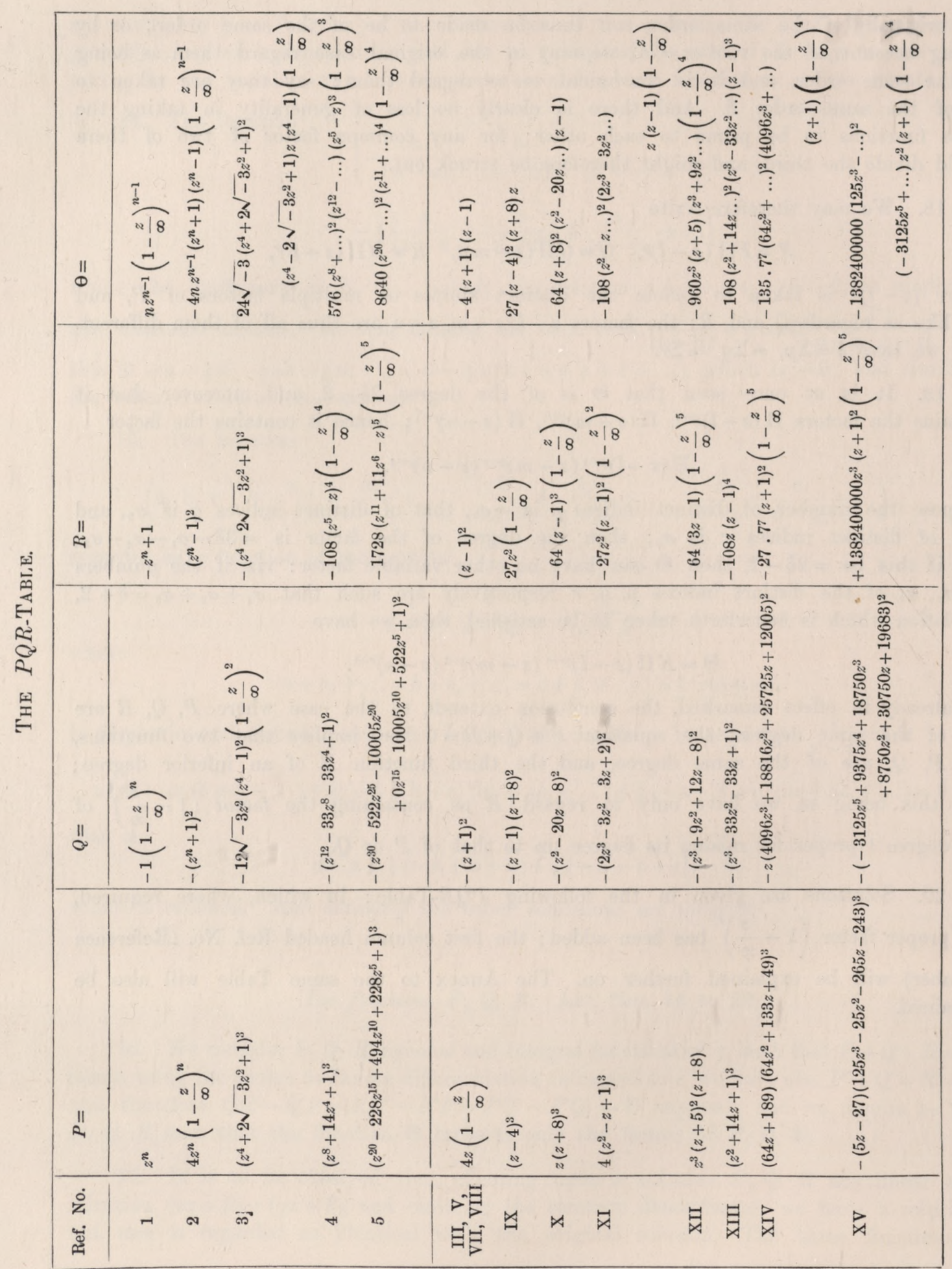

:

ॠ.

형

ष

음

푸

总

ต

s.

ऽ్

可

ฮु

훙

$\geqslant$ है

$\not$ ס

త్ ฐँ

弐

보오

ㅍ

क⿺

$\exists$

‡

ㅖㅛ

루유

通

हर

0

2

क

융 क

吾

घ

믐

ఘึ 쇼

요

ธै แั

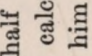

등

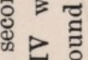
- $\forall$ 


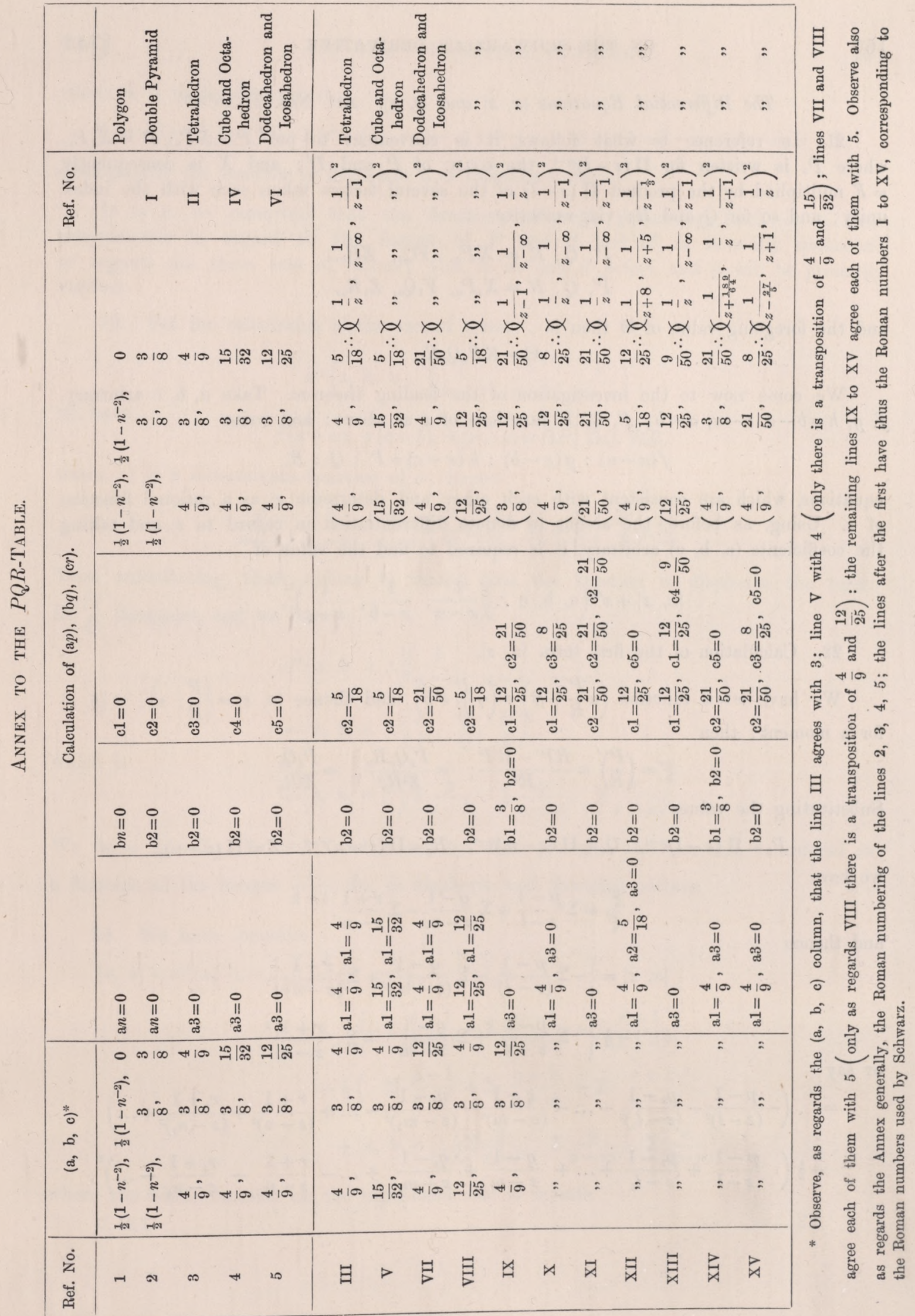


The Differential Equations $\{x, z\}$ and $\{s, x\}$. Art. Nos. 21 to 45.

21. In reference to what follows, it is convenient to put $P=X P_{0}, P^{\prime}=X_{1} P_{0}$, where $P_{0}$ is written for $\Pi(z-l)^{p-1}$, the G.C.M. of $P$ and $P^{\prime}$; and $X$ is consequently $=F$ multiplied by the product $\Pi(z-l)$ of the several factors taken each with the index unity; and so for $Q$ and $R$ : viz. we write

$$
\begin{aligned}
& P, Q, R=X P_{0}, \quad Y Q_{0}, Z R_{0}, \\
& P^{\prime}, Q^{\prime}, R^{\prime}=X_{1} P_{0}, Y_{1} Q_{0}, Z_{1} R_{0},
\end{aligned}
$$

and the foregoing value of $\Theta$ then is

$$
\Theta=K P_{0} Q_{0} R_{0} .
$$

We come now to the investigation of the leading theorem. Take $a, b, c$ arbitrary, $f, g, h=b-c, c-a, a-b ; P, Q, R$ functions of $z$ as above; and write

$$
f(x-a): g(x-b): h(x-c)=P: Q: R,
$$

equations, which are consistent with each other and determine $x$ as a rational function of $z$. Using, as before, the accent to denote differentiation in regard to $z$, and taking the coefficients $(a, b, c)$ arbitrary, it is required to find the value of

$$
\{x, z\}+x^{\prime 2}\left(\mathrm{a}, \mathrm{b}, \mathrm{c} \therefore \gamma \frac{1}{x-a}, \frac{1}{x-b}, \frac{1}{x-c}\right)^{2} .
$$

22. Calculation of the first term $\{x, z\}$.

We have $x=\mathrm{a}$ function $\left(\alpha \frac{P}{R}+\beta\right) \div\left(\gamma \frac{P}{R}+\delta\right)$, and thence $\{x, z\}=\left\{\frac{P}{R}, z\right\},=\{\xi, z\}$ for a moment; then

$$
\xi^{\prime}=\left(\frac{P}{R}\right)^{\prime}=\frac{R P^{\prime}-R^{\prime} P}{R^{2}},=\frac{P_{0} Q_{0} R_{0}}{Z^{2} R_{0}{ }^{2}},=\frac{P_{0} Q_{0}}{Z^{2} R_{0}} .
$$

Substituting the values

$$
P_{0}=\Pi(z-l)^{p-1}, \quad Q_{0}=\Pi(z-m)^{q-1}, \quad R_{0}=\Pi(z-n)^{r-1}, \quad Z=\Pi(z-n),
$$

we have

and thence

$$
\frac{\xi^{\prime \prime}}{\xi^{\prime}}=\Sigma \frac{p-1}{z-l}+\Sigma \frac{q-1}{z-m}-\Sigma \frac{r+1}{z-n}
$$

or say

$$
\begin{array}{r}
\{x, z\}=\left\{-\Sigma \frac{p-1}{(z-l)^{2}}-\Sigma \frac{q-1}{(z-m)^{2}}+\Sigma \frac{r+1}{(z-n)^{2}}\right\} \\
-\frac{1}{2}\left\{\Sigma \frac{p-1}{z-l}+\Sigma \frac{q-1}{z-m}-\Sigma \frac{r+1}{z-n}\right\}^{2} ;
\end{array}
$$

$$
\begin{gathered}
=\left(-\frac{p-1}{(z-l)^{2}}-\frac{p_{1}-1}{\left(z-l_{1}\right)^{2}}-\ldots-\frac{q-1}{(z-m)^{2}}-\frac{q_{1}-1}{\left(z-m_{1}\right)^{2}}-\ldots+\frac{r+1}{(z-n)^{2}}+\frac{r_{1}+1}{\left(z-n_{1}\right)^{2}}+\ldots\right) \\
-\frac{1}{2}\left(\frac{p-1}{z-l}+\frac{p_{1}-1}{z-l_{1}}+\ldots+\frac{q-1}{z-m}+\frac{q_{1}-1}{z-m_{1}}+\ldots-\frac{r+1}{z-n}-\frac{r_{1}+1}{z-n_{1}}-\ldots\right)^{2},
\end{gathered}
$$


where it is to be observed that

$$
\Sigma(p-1)+\Sigma(q-1)-\Sigma(r+1), \quad=\delta-\sigma_{1}+\delta-\sigma_{2}-\left(\delta+\sigma_{3}\right)=\delta-\sigma_{1}-\sigma_{2}-\sigma_{3}=-2 ;
$$

consequently the function is diaphoric, and therefore curtate.

It is to be remarked that the function, although presenting itself in a form unsymmetric in regard to the factors of $P$ and $Q$, and of $R$, is really symmetric as regards the three sets of factors; this is obvious $\grave{a}$ priori, and it will be presently verified.

23. For the calculation of the second term

we have

$$
x^{\prime 2}\left(a, b, c \therefore \gamma \frac{1}{x-a}, \frac{1}{x-b}, \frac{1}{x-c}\right)^{2},
$$

$$
f(x-a), g(x-b), h(x-c)=\Omega P, \Omega Q, \Omega R,
$$

where $\Omega$ is a determinate function of $z$; hence

$$
\frac{x^{\prime}}{x-a}, \frac{x^{\prime}}{x-b}, \frac{x^{\prime}}{x-c}=\frac{P^{\prime}}{P}+\frac{\Omega^{\prime}}{\Omega}, \frac{Q^{\prime}}{Q}+\frac{\Omega^{\prime}}{\Omega}, \frac{R^{\prime}}{R}+\frac{\Omega^{\prime}}{\Omega} .
$$

Then substituting these values, by reason that the function is diaphoric, the terms in $\frac{\Omega^{\prime}}{\Omega}$ disappear, and we have

which is

$$
\begin{aligned}
& x^{\prime 2}\left(\mathrm{a}, \mathrm{b}, \mathrm{c} \therefore \chi \frac{1}{x-a}, \frac{1}{x-b}, \frac{1}{x-c}\right)^{2} \\
& =\left(\mathrm{a}, \mathrm{b}, \mathrm{c} \therefore\left(\frac{P^{\prime}}{P}, \frac{Q^{\prime}}{Q}, \frac{R^{\prime}}{R}\right)^{2},\right.
\end{aligned}
$$

$$
=\left(\mathrm{a}, \mathrm{b}, \mathrm{c} \therefore \gamma_{\Sigma} \frac{p}{z-l}, \quad \Sigma \frac{q}{z-m}, \quad \Sigma \frac{r}{z-n}\right)^{2} \text {. }
$$

We have $\Sigma p=\Sigma q=\Sigma r,=\delta$ : and hence by what precedes, this function, considered as a function of the inverts $\frac{1}{z-l}$, \&c., is diaphoric, and therefore curtate.

24. We have therefore

$$
\begin{aligned}
\{x, z\}+x^{\prime 2}\left(\mathrm{a}, \mathrm{b}, \mathrm{c} \therefore\left(\frac{1}{x-a},\right.\right. & \left.\frac{1}{x-b}, \frac{1}{x-c}\right)^{2}= \\
& \left\{-\Sigma \frac{p-1}{(z-l)^{2}}-\Sigma \frac{q-1}{(z-m)^{2}}+\Sigma \frac{r+1}{(z-n)^{2}}\right\} \\
- & \frac{1}{2}\left(\Sigma \frac{p-1}{z-l}+\Sigma \frac{q-1}{z-m}-\Sigma \frac{r+1}{z-n}\right)^{2} \\
+ & \left(\mathrm{a}, \mathrm{b}, \mathrm{c} \therefore\left(\Sigma \frac{p}{z-l}, \quad \Sigma \frac{q}{z-m}, \quad \Sigma \frac{r}{z-n}\right)^{2},\right.
\end{aligned}
$$

where the whole function on the right-hand side is curtate.

C. XI. 
25. We have to bring the function on the right-hand side into the reduced form

$$
\frac{a}{(z-\alpha)^{2}}+\ldots+\frac{A}{z-\alpha}+\ldots
$$

for the purpose of getting rid of the non-essential inverts (if any).

We write

$$
\begin{aligned}
\Sigma \frac{p-1}{(z-l)^{2}} & =\frac{p-1}{(z-l)^{2}}+\frac{p_{1}-1}{\left(z-l_{1}\right)^{2}}+\ldots \\
& =\frac{p-1}{(z-l)^{2}}+\Sigma^{\prime} \frac{p_{1}-1}{\left(z-l_{1}\right)^{2}}
\end{aligned}
$$

viz. $z-l$ here denotes any particular factor, and $z-l_{1}$ represents any other factor of the same set; and so in other like cases.

26. The whole coefficient of $\frac{1}{(z-l)^{2}}$ is

$$
-(p-1)-\frac{1}{2}(p-1)^{2}+a p^{2}, \quad=\frac{1}{2}\left(1-p^{2}\right)+a p^{2} ;
$$

an expression which, regarded as a function of a and $p$, is represented by (ap): the parentheses are used only to avoid ambiguity, and are omitted when $p$ is a number, thus $\mathrm{a} 1=\mathrm{a}, \mathrm{a} 2=-\frac{3}{2}+4 \mathrm{a}$, and so in other cases.

27. The whole term in $\frac{1}{z-l}$ comes from

$$
\begin{aligned}
& -\frac{p-1}{z-l}\left(\Sigma^{\prime} \frac{p_{1}-1}{z-l_{1}}+\Sigma \frac{q-1}{z-m}-\Sigma \frac{r+1}{z-n}\right) \\
& +\frac{p}{z-l}\left\{2 \mathrm{a} \Sigma^{\prime} \frac{p_{1}}{z-l}+(-\mathrm{a}-\mathrm{b}+\mathrm{c}) \Sigma \frac{q}{z-m}+(-\mathrm{a}+\mathrm{b}-\mathrm{c}) \Sigma \frac{r}{z-n}\right\},
\end{aligned}
$$

viz. each term such as $\frac{1}{z-l \cdot z-l_{1}}$ is to be replaced by $\frac{1}{l-l_{1}}\left(\frac{1}{z-l}-\frac{1}{z-l_{1}}\right)$, giving rise to the term $\frac{1}{l-l_{1}} \frac{1}{z-l}$, or contributing the term $\frac{1}{l-l_{1}}$ to the coefficient of $\frac{1}{z-l}$. The whole coefficient thus is

$$
\begin{aligned}
= & -(p-1)\left(\Sigma^{\prime} \frac{p_{1}-1}{l-l_{1}}+\Sigma \frac{q-1}{l-m}-\Sigma \frac{r+1}{l-n}\right) \\
& +2 a p \Sigma^{\prime} \frac{p_{1}}{l-l_{1}}+p(-a-b+c) \Sigma \frac{q}{l-m}+p(-a+b-c) \Sigma \frac{r}{l-n} .
\end{aligned}
$$

28. Suppose first that $z-l$ is a multiple factor of $P$, viz. a factor with an index $p$ greater than 1: then, for $z=l$, we have $Q+R=0, Q^{\prime}+R^{\prime}=0$, and thence $\frac{Q^{\prime}}{Q}=\frac{R^{\prime}}{R}$, that is, $\Sigma \frac{q}{l-m}=\Sigma \frac{r}{l-n}$. We have therefore

$$
\begin{gathered}
p(-\mathrm{a}-\mathrm{b}+\mathrm{c}) \Sigma \frac{q}{l-m}+p(-\mathrm{a}+\mathrm{b}-\mathrm{c}) \Sigma \frac{r}{l-n} \\
=-a p\left(\Sigma \frac{q}{l-m}+\Sigma \frac{r}{l-n}\right)
\end{gathered}
$$


moreover, in the top line, the terms $\Sigma \frac{q}{l-m}$ and $-\Sigma \frac{r}{l-n}$ destroy each other. The whole coefficient of $\frac{1}{z-l}$, when $z-l$ is a multiple factor of $P$, thus is

$$
\begin{aligned}
=- & (p-1)\left(\Sigma^{\prime} \frac{p_{1}-1}{l-l_{1}}-\Sigma \frac{1}{l-m}-\Sigma \frac{1}{l-n}\right) \\
& +2 a p \Sigma^{\prime} \frac{p_{1}}{l-l_{1}}-a p\left(\Sigma \frac{q}{l-m}+\Sigma \frac{r}{l-n}\right),
\end{aligned}
$$

a form which is now symmetrical in regard to the inverts $\frac{1}{l-m}$ and $\frac{1}{l-n}$.

29. The value just obtained must be equal to

$$
\left(1-p^{2}+2 \mathrm{a} p^{2}\right)\left(\Sigma^{\frac{1}{2} q-1} \frac{\frac{1}{2} r-1}{l-m}+\Sigma^{\prime} \frac{1}{l-l_{1}}\right)
$$

viz. comparing the two forms and reducing, they will be identical if only

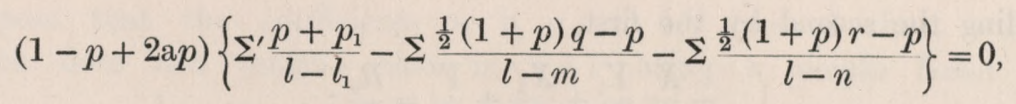

and it can be shown that the function inside the \{\} is in fact $=0$.

30. We have, as before, $\Sigma \frac{q}{l-m}=\Sigma \frac{r}{l-n}$; or writing each of these quantities $=\Phi$, the equation to be verified is

$$
\Sigma^{\prime} \frac{p+p_{1}}{l-l_{1}}=(p+1) \Phi-p \Sigma \frac{1}{l-m}-p \Sigma \frac{1}{l-n} .
$$

We have

$$
\frac{P^{\prime}}{P}=\frac{p}{z-l}+\Sigma^{\prime} \frac{p_{1}}{z-l_{1}},=\frac{X_{1}}{X}
$$

that is,

$$
\begin{aligned}
\Sigma^{\prime} \frac{p_{1}}{l-l_{1}} & =\left[\frac{X_{1}}{X}-\frac{p}{z-l}\right] \text { for } z=l, \\
& =\left[\frac{X_{1}(z-l)-p X}{X(z-l)}\right] .
\end{aligned}
$$

The first derived function of the numerator is $X_{1}^{\prime}(z-l)+X_{1}-p X^{\prime}$, which for $z=l$ is $X_{1}-p X^{\prime}$, which is $=0$; and, for the denominator, it is $X^{\prime}(z-l)+X$, which is also $=0$. Passing to the second derived functions, we find

$$
\Sigma^{\prime} \frac{p_{1}}{z-l_{1}}=\frac{2 X_{1}^{\prime}-p X^{\prime \prime}}{2 X^{\prime}},=\frac{X_{1}^{\prime}-\frac{1}{2} p X^{\prime \prime}}{X^{\prime}} .
$$

From the equation

$$
\frac{X^{\prime}}{X}=\frac{1}{z-l}+\Sigma^{\prime} \frac{1}{z-l}
$$


we find in like manner

$$
\Sigma^{\prime} \frac{1}{l-l_{1}}=\frac{\frac{1}{2} X^{\prime \prime}}{X^{\prime}}
$$

and we thence obtain $(z$ being always $=l$ )

$$
\Sigma^{\prime} \frac{p+p_{1}}{z-l_{1}}=\frac{X_{1}^{\prime}}{X^{\prime}}
$$

so that the equation to be verified becomes

$$
\frac{X_{1}^{\prime}}{X_{1}}=(p+1) \Phi-p \Sigma \frac{1}{l-m}-p \Sigma \frac{1}{l-n} .
$$

31. But from the equation $\Theta,=P Q^{\prime}-P^{\prime} Q,=K P_{0} Q_{0} R_{0}$, we find $X Y_{1}-X_{1} Y=K R_{0}$, and then, differentiating, $X Y_{1}^{\prime}+X^{\prime} Y_{1}-X_{1}^{\prime} Y-X_{1} Y^{\prime}=K R_{0}^{\prime}$ : writing in these equations $z=l$, they become

$$
\begin{aligned}
-X_{1} Y & =K R_{0}, \\
X^{\prime} Y_{1}-X_{1}^{\prime} Y-X_{1} Y^{\prime} & =K R_{0},
\end{aligned}
$$

so that, dividing the second by the first,

$$
-\frac{X^{\prime}}{X_{1}} \frac{Y_{1}}{Y}+\frac{X_{1}^{\prime}}{X_{1}}+\frac{Y^{\prime}}{Y}=\frac{R_{0}^{\prime}}{R_{0}}
$$

or, recollecting that $X_{1}=p X^{\prime}$ and $\frac{Y_{1}}{Y}=\frac{Q^{\prime}}{Q}$, we have

that is,

$$
\frac{X_{1}^{\prime}}{X^{\prime}}=p\left(\frac{R_{0}^{\prime}}{R_{0}}-\frac{Y^{\prime}}{Y}\right)+\frac{Q^{\prime}}{Q},
$$

$$
\begin{aligned}
\frac{X_{1}^{\prime}}{X^{\prime}} & =p\left(\Sigma \frac{r-1}{l-n}-\Sigma \frac{1}{l-m}\right)+\Sigma \frac{q}{l-m}, \\
& =(p+1) \Phi-p \Sigma \frac{1}{l-m}-p \Sigma \frac{1}{l-n},
\end{aligned}
$$

the required relation.

32. The result is that, $z-l$ being a multiple factor of $P$, the coefficient of the term $\frac{1}{z-l}$ is

$$
\begin{aligned}
& =\left(1-p^{2}+2 \mathrm{a} p^{2}\right)\left\{\Sigma \frac{\frac{1}{2} q-1}{l-m}+\Sigma \frac{\frac{1}{2} r-1}{l-n}-\Sigma^{\prime} \frac{1}{l-l_{1}}\right\}, \\
& =2(\mathrm{a} p)\left[\frac{1}{2}\left(\frac{Q^{\prime}}{Q}+\frac{R^{\prime}}{R}\right)-\frac{Y^{\prime}}{Y}-\frac{Z^{\prime}}{Z}-\frac{1}{2} \frac{X^{\prime \prime}}{X^{\prime}}\right] .
\end{aligned}
$$

33. In the case where $z-l$ is a simple factor of $P$ we have $p=1$, and the coefficient is

$$
\begin{aligned}
& =2 \mathrm{a} \Sigma^{\prime} \frac{p_{1}}{l-l_{1}}+(-\mathrm{a}-\mathrm{b}+\mathrm{c}) \Sigma \frac{q}{l-m}+(-\mathrm{a}+\mathrm{b}-\mathrm{c}) \Sigma \frac{r}{l-n}, \\
& =\mathrm{a}\left(2 \Sigma^{\prime} \frac{p_{1}}{l-l_{1}}-\Sigma \frac{q}{l-m}-\Sigma \frac{r}{l-n}\right)-(\mathrm{b}-\mathrm{c})\left(\Sigma \frac{q}{l-m}-\Sigma \frac{r}{l-n}\right) .
\end{aligned}
$$


34. Of course the formulæ for the coefficients of $\frac{1}{(z-l)^{2}}$ and $\frac{1}{z-l}$ give at once, by a mere change of letters, those for the coefficients of $\frac{1}{(z-m)^{2}}, \frac{1}{z-m}$, and $\frac{1}{(z-n)^{2}}, \frac{1}{z-n} ;$ and the function in question,

$$
\{x, z\}+x^{2}\left(\mathrm{a}, \mathrm{b}, \mathrm{c} \therefore \gamma \frac{1}{x-a}, \frac{1}{x-b}, \frac{1}{x-c}\right)^{2},
$$

is now obtained in the required form

$$
=\frac{(\mathrm{a} p)}{(z-l)^{2}} \ldots+\frac{(\mathrm{b} q)}{(z-m)^{2}} \ldots+\frac{(\mathrm{c} r)}{(z-n)^{2}} \ldots+\frac{A}{z-l} \ldots+\frac{B}{z-m} \ldots+\frac{C}{z-n} \ldots
$$

where $(\mathrm{a} p)$ denotes $\frac{1}{2}\left(1-p^{2}\right)+a p^{2}$, and the like for $(\mathrm{b} q)$ and $(\mathrm{c} r)$; and where, $z-l$ being a multiple factor of $P$, the coefficient $A$ contains the factor (ap); and similarly for $B$ and $C$.

35. Suppose that the coefficients $a, b, c$ are no one of them $=0$; we have al, =a, which does not vanish; that is, $z-l$ being a simple factor of $P$, the expression contains $\frac{1}{(z-l)^{2}}$, or the invert $\frac{1}{z-l}$ is essential : and similarly, $z-m$ being a simple factor of $Q$, or $z-n$ a simple factor of $R$, the inverts $\frac{1}{z-m}$ and $\frac{1}{z-n}$ are essential. But for $z-l$ a multiple factor of $P$, the coefficient (ap) of the term $\frac{1}{(z-l)^{2}}$ may vanish, viz. this will be the case if $a=\frac{1}{2}\left(1-\frac{1}{p^{2}}\right)$; and, when this is so, the coefficient $A$ of the corresponding term $\frac{1}{z-l}$ also vanishes; that is, $\frac{1}{z-l}$ is a nonessential invert. And similarly for any multiple factor $z-m$ of $Q$ or $z-n$ of $R$, the invert $\frac{1}{z-m}$ or $\frac{1}{z-n}$ may be non-essential.

36. If $P, Q, R$ contain each of them only multiple factors of the same index, say of the indices $p, q, r$ for the three functions respectively, viz. if the functions are $F(\Pi(z-l))^{p}, G(\Pi(z-m))^{q}, H(\Pi(z-n))^{r}$, the result contains only the six terms written down: and then, if $\mathrm{a}, \mathrm{b}, \mathrm{c}$ are $=\frac{1}{2}\left(1-\frac{1}{p^{2}}\right), \frac{1}{2}\left(1-\frac{1}{q^{2}}\right), \frac{1}{2}\left(1-\frac{1}{r^{2}}\right)$ respectively the result is $=0$ : viz. we then have

$$
\{x, z\}+x^{\prime 2}\left(a, b, c \therefore \gamma \frac{1}{x-a}, \frac{1}{x-b}, \frac{1}{x-c}\right)=0,
$$

or we in fact have, for the values in question of $a, b, c$, a solution

$$
f(x-a): g(x-b): h(x-c)=P: Q: R
$$

of this differential equation of the third order. 
37. The reasoning applies directly to lines $2,3,4,5$ of the $P Q R$-Table: and with a slight variation to line 1 ; viz. here the factors of $R\left(=-1+z^{n}\right)$ are all simple factors, but in virtue of $\mathrm{c}=0$ and $\mathrm{a}=\mathrm{b}$, the corresponding inverts disappear, and, the other inverts also disappearing, the value of the function is $=0$. Hence lines 1, 2, $3,4,5$ of the $P Q R$-Table give each of them a result $=0$, for the values of $(\mathrm{a}, \mathrm{b}, \mathrm{c})$ appearing by the table itself, and shown explicitly in the corresponding line of the Annex.

Thus line 3 shows that the function $x$, determined by

$$
f(x-a): g(x-b): h(x-c)=\left(z^{4}+2 \sqrt{-3} z^{2}+1\right)^{3}:-12 \sqrt{-3}\left(z^{5}-z\right)^{2}:-\left(z^{4}-2 \sqrt{-3} z^{2}+1\right)^{3},
$$

satisfies

$$
\{x, z\}+x^{\prime 2}\left(\frac{4}{9}, \frac{3}{8}, \frac{4}{9} \therefore \gamma \frac{1}{x-a}, \frac{1}{x-b}, \frac{1}{x-c}\right)^{2}=0,
$$

and so for any other of the five lines.

38. The indices of the factors of $P, Q, R$ may be such that, for proper values of the coefficients $a, b, c$, there are in all only three essential inverts, say $\frac{1}{z-a_{1}}$, $\frac{1}{z-b_{1}}, \frac{1}{z-c_{1}}$, belonging to the three functions $P, Q, R$ respectively, or it may be two, or three, of them to the same function. When this is so, the function of these inverts is, by what precedes, a curtate function, and it is consequently a function

$$
\left(\mathrm{a}_{1}, \mathrm{~b}_{1}, \mathrm{c}_{1} \therefore \gamma \frac{1}{z-a_{1}}, \frac{1}{z-b_{1}}, \frac{1}{z-c_{1}}\right)^{2},
$$

where $a_{1}, b_{1}, c_{1}$ are the values of the three which do not vanish in the series of expressions (ap), (bq), (cr).

The remaining lines (III, V, VII, VIII) and IX to XV of the $P Q R$-Table give such values of $P, Q, R$, the values of $(\mathrm{a}, \mathrm{b}, \mathrm{c})$; and the calculation of the values of $\left(a_{1}, b_{1}, c_{1}\right)$ is shown by the corresponding lines of the Annex. And we have thus values of $x$ determined by the equations

and giving

$$
f(x-a): g(x-b): h(x-c)=P: Q: R,
$$

$\{x, z\}+x^{\prime 2}\left(\mathrm{a}, \mathrm{b}, \mathrm{c} \therefore\left(\frac{1}{x-a}, \frac{1}{x-b}, \frac{1}{x-c}\right)^{2}=\left(\mathrm{a}_{1}, \mathrm{~b}_{1}, \mathrm{c}_{1} \therefore\left(\frac{1}{z-a_{1}}, \frac{1}{z-b_{1}}, \frac{1}{z-c_{1}}\right)^{2}\right.\right.$.

39. For instance, from line IX we have

$$
f(x-a): g(x-b): h(x-c)=(z-4)^{3}:-(z-1)(z+8)^{2}: 27 z^{2}\left(1-\frac{z}{\infty}\right),
$$

the values of $(\mathrm{a}, \mathrm{b}, \mathrm{c})$ are $\frac{4}{9}, \frac{3}{8}, \frac{12}{25}$; and since $P, Q, R$ contain factors with the exponents $3 ; 1,2$; and 1,2 respectively, the coefficients which present themselves on the right-hand side are

$$
\mathrm{a} 3 ; \mathrm{b} 1, \mathrm{~b} 2 ; \mathrm{c} 1, \mathrm{c} 2 \text {, }
$$


which are

$$
=0 ; \frac{3}{8}, 0 ; \frac{12}{25}, \frac{21}{50} \text { respectively. }
$$

Hence writing $a_{1}, b_{1}, c_{1}=\frac{3}{8}, \frac{12}{25}, \frac{21}{50}$, the corresponding inverts are $\frac{1}{z-1}, \frac{1}{z-\infty}$, $\frac{1}{z}$; and the result is

$$
\{x, z\}+x^{\prime 2}\left(\frac{4}{9}, \frac{3}{8}, \frac{12}{25} \therefore \gamma \frac{1}{x-a}, \frac{1}{x-b}, \frac{1}{x-c}\right)^{2}=\left(\frac{3}{8}, \frac{12}{25}, \frac{21}{50} \therefore \gamma \frac{1}{z-1}, \frac{1}{z-\infty}, \frac{1}{z}\right)^{2} .
$$

40. It is hardly necessary to remark that an expression

in fact denotes

$$
\left(\mathrm{a}_{1}, \mathrm{~b}_{1}, \mathrm{c}_{1} \therefore \gamma \frac{1}{z-a_{1}}, \frac{1}{z-b_{1}}, \frac{1}{z-\infty}\right)^{2}
$$

$$
\frac{a_{1}}{\left(z-a_{1}\right)^{2}}+\frac{b_{1}}{\left(z-b_{1}\right)^{2}}+\frac{-a_{1}-b_{1}+c_{1}}{\left(z-a_{1}\right)\left(z-b_{1}\right)} \text {. }
$$

The particular form of the $z$ inverts is immaterial; we could by a general linear transformation upon the $z$ make them to be $\frac{1}{z-a_{1}}, \frac{1}{z-b_{1}}, \frac{1}{z-c_{1}}$ with the $\left(a_{1}, b_{1}, c_{1}\right)$ arbitrary; or we can give to the $a_{1}, b_{1}, c_{1}$ any particular values we please: there would be a propriety in making the inverts to be in every case (as in the foregoing example) $\frac{1}{z}, \frac{1}{z-\infty}, \frac{1}{z-1}$; but the numerical work would be troublesome, and it is not worth while to effect it.

41. The conclusion is that lines (III, V, VII, VIII) and IX to XV of the $P Q R$-Table, give, for determinate values of $(a, b, c)$ and $\left(a_{1}, b_{1}, c_{1}\right)$, solutions

of the equation

$$
f(x-a): g(x-b): h(x-c)=P: Q: R
$$

$$
\{x, z\}+x^{\prime 2}\left(\mathrm{a}, \mathrm{b}, \mathrm{c} \therefore \chi \frac{1}{x-a}, \frac{1}{x-b}, \frac{1}{x-c}\right)^{2}=\left(\mathrm{a}_{1}, \mathrm{~b}_{1}, \mathrm{c}_{1} \therefore \gamma \frac{1}{z-a_{1}}, \frac{1}{z-b_{1}}, \frac{1}{z-c_{1}}\right)^{2},
$$

where $a, b, c, a_{1}, b_{1}, c_{1}$ are or can be made arbitrary, but without any real gain of generality herein. This is the Differential Equation $\{x, z\}$.

42. Recurring to the results from the Arabic lines of the $P Q R$-Table, but for convenience writing $s$ instead of $z$, we have

$$
f(x-a): g(x-b): h(x-c)=P: Q: R,
$$

where $P, Q, R$ are now functions of $s$, a solution of

$$
\{x, s\}+\left(\frac{d x}{d s}\right)^{2}\left(a, b, c \therefore \gamma \frac{1}{x-a}, \frac{1}{x-b}, \frac{1}{x-c}\right)^{2}=0 .
$$

But we have

$$
\{s, x\}=-\left(\frac{d s}{d x}\right)^{2}\{x, s\}
$$


and the foregoing is therefore a solution of

$$
\{s, x\}=\left(\mathrm{a}, \mathrm{b}, \mathrm{c} \therefore \gamma \frac{1}{x-a}, \frac{1}{x-b}, \frac{1}{x-c}\right)^{2},
$$

a differential equation of the third order. This is the Differential Equation $\{s, x\}$.

43. From the Roman lines, if we assume

$$
f(x-a): g(x-b): h(x-c)=\mathfrak{B}: \mathfrak{Q}: \Re,
$$

where $\mathfrak{i}, \mathbb{Q}, \Re$ are functions of $z$, not the same functions that $P, Q, R$ are of $s$, since they belong to a different line of the Table: we have, as before,

$$
\{x, z\}+\left(\frac{d x}{d z}\right)^{2}\left(\mathrm{a}, \mathrm{b}, \mathrm{c} \therefore \gamma \frac{1}{x-a}, \frac{1}{x-b}, \frac{1}{x-c}\right)^{2}=\left(\mathrm{a}_{1}, \mathrm{~b}_{1}, \mathrm{c}_{1} \therefore \gamma \frac{1}{z-a_{1}}, \frac{1}{z-b_{1}}, \frac{1}{z-c_{1}}\right)^{2} \text {. }
$$

44. We may combine any such result with a properly selected result of the preceding system, the two results being such that $(a, b, c)$ have the same values in each of them. (See as to this the foot-note referring to the Annex to the $P Q R$ Table.) The last equation then becomes

or since

$$
\{x, z\}+\left(\frac{d x}{d z}\right)^{2}\{s, x\}=\left(\mathrm{a}_{1}, \mathrm{~b}_{1}, \mathrm{c}_{1} \therefore\left(\frac{1}{z-a_{1}}, \frac{1}{z-b_{1}}, \frac{1}{z-c_{1}}\right)^{2},\right.
$$

this is

$$
\{x, z\}+\left(\frac{d x}{d z}\right)^{2}\{s, x\}=\{s, z\}
$$

$$
\{s, z\}=\left(\mathrm{a}_{1}, \mathrm{~b}_{1}, \mathrm{c}_{1} \therefore \gamma \frac{1}{z-a_{1}} \cdot \frac{1}{z-b_{1}}, \frac{1}{z-c_{1}}\right)^{2},
$$

the corresponding relation between $s, z$ being of course obtained by the elimination of $x$ from the two sets of equations

$f(x-a): g(x-b): h(x-c)=P: Q: R$, and $f(x-a): g(x-b): h(x-c)=\mathfrak{B}: \mathfrak{Q}: \Re$;

viz. the required relation is

$$
P: Q: R=\Re: \mathbb{Q}: \Re,
$$

where $P, Q, R$ are functions of $s ; \mathfrak{B}, \mathfrak{Q}, \Re$ functions of $z$; and, in virtue of

$$
P+Q+R=0, \quad B+Q+\Re=0,
$$

the relations are equivalent to a single equation between $z$ and $s$. And writing finally $x$ in place of $z$, that is, now considering $\mathfrak{B}, \mathfrak{\Omega}, \Re$ as functions of $x$, we have

as a solution of

$$
\Re: \mathscr{Q}: \Re=P: Q: R
$$

$$
\{s, x\}=\left(a_{1}, b_{1}, c_{1} \therefore \gamma \frac{1}{x-a_{1}}, \frac{1}{x-b_{1}}, \frac{1}{x-c_{1}}\right)^{2},
$$

a differential equation of the third order of the foregoing form, $\{s, x\}=$ given function of $x$, but with different values of the coefficients, $\left(a_{1}, b_{1}, c_{1}\right)$ instead of $(a, b, c)$. 
45. It thus appears that there are in all 16 sets of values of $(a, b, c)$, for which the equation is solved, viz. the 16 sets of values are shown in the righthand column of the Annex. For greater clearness I exhibit the integral equations as follows:

\begin{tabular}{|c|c|c|c|c|c|}
\hline \multirow{3}{*}{$\begin{array}{l}1 \\
\mathrm{I}\end{array}$} & Functions of $x$. & \multicolumn{3}{|c|}{ Functions of $s$. } & \multirow[b]{2}{*}{ Polygon } \\
\hline & $f(x-a): g(x-b): h(x-c)$ & & $Q$ & (1) & \\
\hline & , & $=$ & , & $(2)$ & Double Pyramid \\
\hline II & , & $=$ & , & (3) & Tetrahedron \\
\hline III & $4 x:-(x+1)^{2}:(x-1)^{2}$ & $=$ & $"$ & (3) & , \\
\hline IV & $f(x-a): g(x-b) \quad: h(x-c)$ & $=$ & , & (4) & Cube and Octahedron \\
\hline $\mathrm{V}$ & $(x-1)^{2}:-(x+1)^{2}: 4 x$ & $=$ & , & (4) & $"$ \\
\hline VI & $f(x-a): g(x-b): h(x-c)$ & $=$ & " & (5) & Dodecahedron and Icosahedron \\
\hline VII & $4 x \quad:-(x+1)^{2}:(x-1)^{2}$ & $=$ & ", & $(5)$ & , \\
\hline VIII & $(x-1)^{2}:-(x+1)^{2}: 4 x$ & $=$ & ", & $(5)$ & ", \\
\hline IX & $P \quad: Q \quad: R \quad(\mathrm{IX})$ & $=$ & $"$ & $(5)$ & , \\
\hline $\mathrm{X}$ & $(\mathrm{X})$ & $=$ & $"$ & (5) & ", \\
\hline $\mathrm{XI}$ & $(\mathrm{XI})$ & $=$ & " & $(5)$ & , \\
\hline $\mathrm{XII}$ & (XII) & $=$ & ", & $(5)$ & , \\
\hline $\mathrm{XIII}$ & (XIII) & $=$ & , & $(5)$ & ", \\
\hline XIV & (XIV) & $=$ & , & (5) & , \\
\hline $\mathrm{XV}$ & $(\mathrm{XV})$ & $=$ & , & $(5)$ & ", \\
\hline
\end{tabular}

The values of the $P, Q, R$ as functions of $x$, or of $s$, are taken out of the $P Q R$-Table: only in the lines III, V, VII, VIII, where $P, Q, R$ are given as

$$
=4 z, \quad-(z+1)^{2}, \quad(z-1)^{2},
$$

and where, as regards $\mathrm{V}$ and VIII, there is a transposition of $P$ and $R$, I have inserted the actual values of the $x$-functions. (See as to this the foot-note referring to the Annex.)

The Schwarzian Theory. Art. Nos. 46 to 62.

46. Considering the foregoing equation

$$
\{s, x\}=\left(\mathrm{a}_{1}, \mathrm{~b}_{1}, \mathrm{c}_{1} \therefore \chi \frac{1}{x-a_{1}}, \frac{1}{x-b_{1}}, \frac{1}{x-c_{1}}\right)^{2}
$$

as a particular case of the equation $\{s, x\}=$ Rational function of $x,=R(x)$ suppose, then we have in 1 , I, II, IV, VI solutions of the form $x$ =Rational function of $s$.

C. XI. 
Consider, in general, a solution of this form, $x=F(s)$ a rational function of $s$ : then $s$ is an irrational function of $x$, and if $s_{1}, s_{2}$ are any two of its values, $\left\{s_{1}, x\right\}=R(x)$, $\left\{s_{2}, x\right\}=R(x)$; that is, $\left\{s_{2}, x\right\}=\left\{s_{1}, x\right\}$, and therefore (ante, No. 7) $s_{2}=\frac{a s_{1}+b}{c s_{1}+d}$. And then $x=F\left(s_{2}\right)=F\left(\frac{a s_{1}+b}{c s_{1}+d}\right),=F\left(s_{1}\right):$ viz. $F(s)$ is a rational function of $s$, transformable into itself by the transformation $s$ into $\frac{a s+b}{c s+d}$ : and it is moreover clear that between any two roots $s$ whatever of the equation $x=F^{\prime}(s)$ there exists a homographic relation of the form in question. Further, it is clear that these homographic transformations form a group; and consequently that $F(s)$ is a rational function of $s$, transformable into itself by the several homographic transformations of a group of such transformations: viz. taking $x$ to be a rational function of $s$, it is only in the case $x=F(s)$, a function of the form in question, that $\{s, x\}$ can be equal to a rational function of $x$.

47. We may, in any equation between $x$ and $s$, consider these as imaginary variables $p+q i$ and $u+v i$ respectively; considering then $(p, q)$ and $(u, v)$ as rectangular coordinates of points in different planes, we have a first plane the locus of the points $x$, and a second plane the locus of the points $s$ : there is between the two planes a correspondence which is in fact the correspondence of conformable figures: to the infinitesimal element $d x$ drawn from a point $x$ of the first figure corresponds an infinitesimal element $d s$ drawn from the corresponding point $s$ of the second figure, these elements being in general connected by an equation of the form $d s=(a+b i) d x$, where $a$ and $b$ are functions of $x$ or $s$; and this signifies that, to obtain the pencil of infinitesimal elements or radii $d s$ proceeding in different directions from the point $s$, we alter in a determinate ratio the absolute lengths of the infinitesimal elements or radii proceeding from the corresponding point $x$, and rotate the pencil through a determinate angle: this ratio and angle of rotation, or say, the Auxesis and the Streblosis, being of course variable from point to point. Or, what comes to the same thing, if $d x$ and $d_{1} x$ be consecutive elements of the path of the point $x$, and $d s, d_{1} s$ the corresponding consecutive elements of the path of the point $s$, then the ratio of the lengths of the elements $d x, d_{1} x$ is equal to that of the lengths of the elements $d s, d_{1} s$; and the mutual inclination of the first pair of elements is equal to that of the second pair of elements. In particular, if at any point the path of $x$ is a curved line without abrupt change of direction, then at the corresponding point the path of $s$ is a curved line without abrupt change of direction. In what precedes, we have the relation at ordinary points; but there may be critical corresponding points $(x, s)$, the relation at a critical point between the corresponding elements $d x$, $d s$ being of the form $d s=(a+b i)(d x)^{\lambda}$, ( $\lambda$ a positive integer or fraction): here the angle between two elements $d s$ is $=\lambda$ times that between the two elements $d x$; or, if the path of the point $x$ through the critical point is without abrupt change of direction, say if the angle between the two consecutive elements is the flat angle $\pi$, then the angle between the two consecutive elements $d s$ is $=\lambda \pi$ : viz. there may be in the path of the point $s$ an abrupt change of direction. 
48. I consider the foregoing equation $\{s, x\}=R(x)$, where $R(x)$ is a rational function, and is now taken to be a real function of $x$ : we may assume $s^{\prime}=i \rho^{\prime} \theta^{\prime} e^{i \theta}$, where the accents denote differentiation in regard to $x$, and where $\rho^{\prime}, \theta$, and therefore also $\theta^{\prime}$, are real functions of $x$. We have

and thence

$$
\frac{s^{\prime \prime}}{s^{\prime}}=\frac{\rho^{\prime \prime}}{\rho^{\prime}}+\frac{\theta^{\prime \prime}}{\theta^{\prime}}+i \theta^{\prime}
$$

$$
\begin{aligned}
& \frac{s^{\prime \prime \prime}}{s^{\prime}}-\left(\frac{s^{\prime \prime}}{s^{\prime}}\right)^{2}=\frac{\rho^{\prime \prime \prime}}{\rho^{\prime}}-\left(\frac{\rho^{\prime \prime}}{\rho^{\prime}}\right)^{2}+\frac{\theta^{\prime \prime \prime}}{\theta^{\prime}}-\left(\frac{\theta^{\prime \prime}}{\theta^{\prime}}\right)^{2}+i \theta^{\prime \prime} \\
& -\frac{1}{2}\left(\frac{s^{\prime \prime}}{s^{\prime}}\right)^{2}=-\frac{1}{2}\left(\frac{\rho^{\prime \prime}}{\rho^{\prime}}\right)^{2}-\frac{1}{2}\left(\frac{\theta^{\prime \prime}}{\theta^{\prime}}\right)^{2}+\frac{1}{2} \theta^{\prime 2}-\frac{\rho^{\prime \prime} \theta^{\prime \prime}}{\rho^{\prime} \theta^{\prime}}-i \theta^{\prime \prime}-i \frac{\rho^{\prime \prime} \theta^{\prime}}{\rho^{\prime}}
\end{aligned}
$$

and thence

$$
\{s, x\}=\{\rho, x\}+\{\theta, x\}+\frac{1}{2} \theta^{\prime 2}-\frac{\rho^{\prime \prime} \theta^{\prime \prime}}{\rho^{\prime} \theta^{\prime}}-i \frac{\rho^{\prime \prime} \theta^{\prime}}{\rho^{\prime}} .
$$

Putting this $=R(x)$, and assuming that $x$ is real, we have

$$
\{\rho, x\}+\{\theta, x\}+\frac{1}{2} \theta^{\prime 2}-\frac{\rho^{\prime \prime} \theta^{\prime \prime}}{\rho^{\prime} \theta^{\prime}}=R(x) ; \quad 0=i \frac{\rho^{\prime \prime} \theta^{\prime}}{\rho^{\prime}} .
$$

The last equation gives $\rho^{\prime \prime} \theta^{\prime}=0$, that is, $\theta^{\prime}=0$, which gives $s^{\prime}=0$, and may be disregarded; or else $\rho^{\prime \prime}=0$, therefore $\rho^{\prime}$, a real constant, $=\gamma$ suppose, and $\{\rho, x\}=0$ : hence for the solution of the equation $\{s, x\}=R(x)$, we have $s^{\prime}=i \gamma \theta^{\prime} e^{i \theta}, \theta$ a real quantity determined by $\{\theta, x\}+\frac{1}{2} \theta^{\prime 2}=R(x)$ : and then, integrating the equation for $s^{\prime}$, we have $s=\alpha+\beta i+\gamma e^{i \theta}, \alpha, \beta, \gamma$ real constants.

49. The conclusion is that, if $\{s, x\}=R(x)$, a real function of $x$, and if $x$ be real, that is, if the point $x$ move along a right line (say the $x$-line), then $s=\alpha+\beta i+\gamma e^{i \theta}$ ( $\theta$, and the constants $\alpha, \beta, \gamma$, being real), that is, the point $s$ moves in a circle, coordinates of the centre $\alpha, \beta$, and radius $=\gamma$.

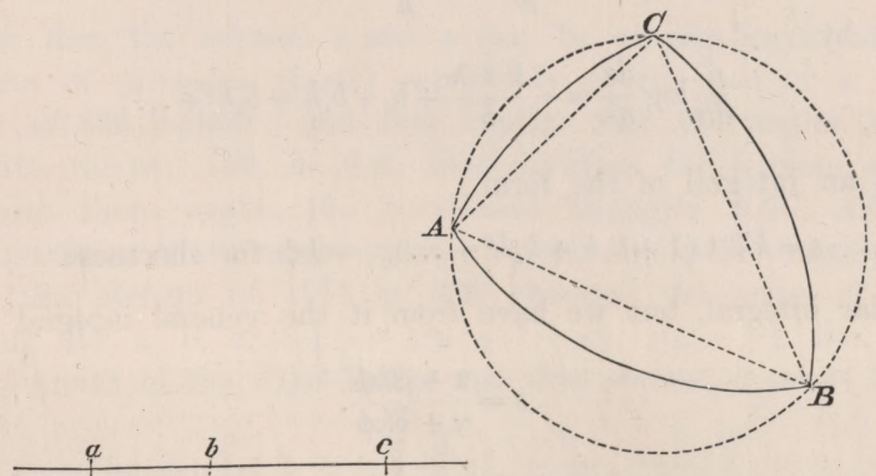

50. Suppose $a, b, c$ are any real values of $x$ representing points $a, b, c$ on the $x$-line; and $A, B, C$ any given imaginary values of $s$ representing points $A, B, C$ 
in the s-plane: since $\{s, x\}=R(x)$ is a differential equation of the third order, the integral contains three arbitrary constants, and we may imagine these so determined that to the values $x=a, b, c$ shall correspond the values $s=A, B, C$ respectively.

If there is not on the $x$-line any critical point, as the point $x$ moves continuously along this line the point $s$ will move continuously along a circle, which (inasmuch as $a, b, c$ and $A, B, C$ are corresponding points) must be the circle through the three points $A, B, C^{*}$.

51. If however the points $a, b, c$ are critical points, such that the element $d s$ at the corresponding points $A, B, C$ are equal to multiples of $(d x)^{\lambda},(d x)^{\mu}$, $(d x)^{\nu}$ respectively, then to the flat angles $\pi$ at $a, b, c$ correspond in the path of $s$ the angles $\lambda \pi, \mu \pi, \nu \pi$ at the points $A, B, C$ respectively: and, assuming that $a, b, c$ are the only critical points on the $x$-line, the path of $s$ is made up of the three circular ares $C A, A B, B C$ meeting at angles $\lambda \pi, \mu \pi, \nu \pi$ respectively. The ares are completely determined by these conditions; for supposing the are $B C$ to make with the chord $B C$, at the points $B$ and $C$, the angles $f, f$, and similarly the arcs $C A$ and $A B$ to make with the corresponding chords the angles $g, g$ and $h, h$, then the conditions give $\lambda \pi, \mu \pi, \nu \pi=\angle A+g+h, \angle B+h+f, \angle C+f+g$, where the angles referred to are those of the rectilinear triangle $A B C$ : we have thus the values of $f, g, h$; and the arc $B C$ is the arc on the chord $B C$ meeting it at angles $f, f$ : and the like as regards the $\operatorname{arcs} C A$ and $A B$ respectively.

52. The foregoing equation

$$
\{s, x\}=\left(\mathrm{a}, \mathrm{b}, \mathrm{c} \therefore \chi \frac{1}{x-a}, \frac{1}{x-b}, \frac{1}{x-c}\right)^{2},
$$

where a, b, c have the values $\frac{1}{2}\left(1-\lambda^{2}\right), \frac{1}{2}\left(1-\mu^{2}\right), \frac{1}{2}\left(1-\nu^{2}\right)$, and $\lambda, \mu, \nu$ are real and positive, has $x=a, b, c$ for critical points of the kind in question. In fact, writing $x-a=h$, the equation is of the form

which is satisfied by

$$
\{s, h\}=\frac{\frac{1}{2}\left(1-\lambda^{2}\right)}{h^{2}}+\frac{a_{0}}{h}+a_{1}+a_{2} h+\ldots,
$$

$$
\frac{d}{d h} \log \frac{d s}{d h}=-\frac{1+\lambda}{h}+b_{0}+b_{1} h+b_{2} h^{2}+\ldots ;
$$

we thence obtain an integral of the form

$$
s=k h^{-\lambda}\left(1+k_{1} h+k_{2} h^{2}+\ldots\right), \quad=k \phi \text { for shortness. }
$$

This is a particular integral, but we have from it the general integral

$$
s=\frac{\alpha+\beta k \phi}{\gamma+\delta k \phi} .
$$

* Since there is no critical point on the $x$-line there can be no abrupt change of direction in the path of $s$, that is, the path of $s$ cannot consist of circular ares meeting at an angle: but it is in the text further assumed that the path of $s$ cannot consist of different ares of circle, the one continuing the other without any abrupt change of direction. 
If $A$ be the value of $s$ corresponding to $h=0$, then $\beta=\delta A$, and we find

$$
s=\frac{\alpha+A \delta k \phi}{\gamma+\delta k \phi},=\left(A+\frac{\alpha}{\delta k \phi}\right)\left(1+\frac{\gamma}{\delta k \phi}\right)^{-1},=A+\frac{\alpha-\gamma A}{\delta k} \frac{1}{\phi}+\ldots
$$

viz. reducing $\frac{1}{\phi}$ to its principal term $h^{\lambda}$, and then writing $d s, d x$ for $s-A$, and $h(=x-a)$ respectively, we have $d s=K(d x)^{\lambda}$, or $x=a$ is a critical point with the exponent $\lambda$; and similarly $x=b$ and $x=c$ are critical points with the exponents $\mu$ and $\nu$ respectively.

53. Hence in the equation

$$
\{s, x\}=\left(\mathrm{a}, \mathrm{b}, \mathrm{c} \therefore \gamma \frac{1}{x-a}, \frac{1}{x-b}, \frac{1}{x-c}\right)^{2},
$$

as the point $x$, passing successively through $a, b, c$, describes the $x$-line, the point $s$, passing successively through $A, B, C$, describes the sides $A B, B C, C A$ of the curvilinear triangle $A B C$. To points $x$ indefinitely near the $x$-line correspond points $s$ indefinitely near the boundary $A B, B C, C A$ of the triangle, viz. to points $x$ indefinitely near to and on one side, suppose the upper side, of the $x$-line, correspond the points $s$ indefinitely near to and within the boundary of the triangle: and in like manner to whole series of "the points $x$ on the same upper side of the $x$-line, correspond the whole series of points $s$ inside the triangle.

54. We have attended so far only to one of the points $s$ which correspond to a given point $x$, but considering the set of points $s$ which correspond to the same point $x$, we have in the $s$-plane entire circles forming by their intersections curvilinear triangles $A B C, A B C^{\prime}$, \&c.; we have thus two systems, say $A B C$, \&c., and $A B C^{\prime}$, \&c., of triangles, such that to a point $x$ on the upper side of the $x$-line correspond points $s$, one of them within each of the triangles $A B C$, \&c., and to a point $x$ on the lower side of the $x$-line correspond points $s$, one of them within each of the triangles $A B C^{\prime}, \& c$; ; and so consequently that, to the two half-planes on opposite sides of the $x$-line, correspond the two sets of triangles $A B C, \& c .$, and $A B C^{\prime}, \& c .$, respectively.

55. In order that the relation $s$ and $x$ may be an algebraical one, it is necessary that the two sets of triangles should completely cover, once or a finite number of times, the whole of the $s$-plane: and this implies that the angles $\lambda \pi, \mu \pi, \nu \pi$ have certain determinate values; and, in fact, that dividing the surface of a sphere into triangles, each with these angles, the curvilinear triangles $A B C, A B C^{\prime}$, \&c., are the stereographic projections of these triangles. It was by such considerations as these that Schwarz, in the Memoir of 1873, p. 323, obtained the series of values $\mathrm{I}$ to XV of $\lambda, \mu, \nu$, giving for $\mathrm{a}, \mathrm{b}, \mathrm{c},=\frac{1}{2}\left(1-\lambda^{2}\right), \frac{1}{2}\left(1-\mu^{2}\right), \frac{1}{2}\left(1-\nu^{2}\right)$, the series of values mentioned in the Annex of the $P Q R$-Table: and thus showed $\grave{a}$ priori that the equation

$$
\{s, x\}=\left(\mathrm{a}, \mathrm{b}, \mathrm{c} \therefore \gamma \frac{1}{x-a}, \frac{1}{x-b}, \frac{1}{x-c}\right)^{2}
$$

is algebraically integrable for these values of a, b, c; and only for these values, or for values reducible to them. 
56. As an instance, take the double pyramid form: the integral equation is

$$
f(x-a): g(x-b): h(x-c)=4 s^{n}:-\left(s^{n}-1\right)^{2}:\left(s^{n}+1\right)^{2},
$$

or say

$$
\frac{(c-a)(x-b)}{(a-b)(x-c)}=-\frac{\left(s^{n}-1\right)^{2}}{\left(s^{n}+1\right)^{2}}
$$

or if, for greater simplicity, we assume $a, b, c=1,0, \infty$, this is $x=\frac{\left(s^{n}-1\right)^{2}}{\left(s^{n}+1\right)^{2}}$, or say $-\left(s^{n}-1\right)=\sqrt{x}\left(s^{n}+1\right)$, that is, $s^{n}=\frac{1 \mp \sqrt{x}}{1 \pm \sqrt{x}}$, a solution of the differential equation

$$
\{s, x\}=\left(\frac{3}{8}, \frac{1}{2}\left(1-n^{-2}\right), \frac{3}{8} \therefore \chi \frac{1}{x}, \frac{1}{x-1}, \frac{1}{x-\infty}\right)^{2} .
$$

In particular, if $n=3$, we have $x=\left(\frac{s^{3}-1}{s^{3}+1}\right)^{2}$ or $s^{3}=\frac{1 \mp \sqrt{x}}{1 \pm \sqrt{ } x}$, a solution of

$$
\{s, x\}=\left(\frac{3}{8}, \frac{4}{9}, \frac{3}{8} \therefore \nmid \frac{1}{x}, \frac{1}{x-1}, \frac{1}{x-\infty}\right)^{2} .
$$

57. We have here the spherical surface divided by the equator and three meridians into twelve triangles, each with the angles $\frac{1}{2} \pi, \frac{1}{2} \pi, \frac{1}{3} \pi$ : and then, projecting from the South pole on the plane of the equator, we have the annexed figure of the $s$-plane,

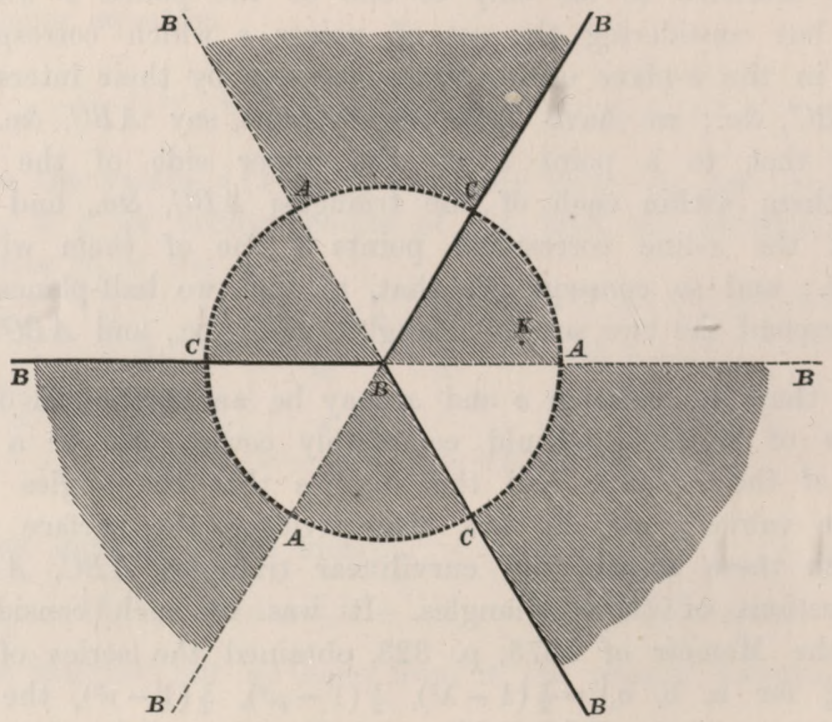

divided into 12 curvilinear triangles, each with these same angles $90^{\circ}, 90^{\circ}, 60^{\circ}$; the plane is divided by the shading into two systems, each of 6 triangles. The figure of the $x$-plane is by the $x$-line divided into two half-planes, one shaded, the other unshaded; and we have on the line the point $c$ at $\infty, a$ at the origin, and $b$ at the distance unity. 
58. Take $x$ real; then, if $x$ is positive and less than $1, s^{3}$ is real and positive, and we have for $s$ the infinite half-lines at the inclinations $0^{\circ}, 120^{\circ}, 240^{\circ}$, while if $x$ is positive and greater than $1, s^{3}$ is real and negative, and we have the infinite half-lines at the inclinations $60^{\circ}, 180^{\circ}, 300^{\circ}$. If $x$ is real and negative, then $s^{3}$ is of the form $\frac{1-k i}{1+k i},=\cos \theta+i \sin \theta$; whence $s$ is of the same form, or the locus of the point $s$ is a circle radius unity. Writing $s^{3}=\frac{1-\sqrt{x}}{1+\sqrt{x}}$, and supposing that the point $x$ moves along the $x$-line from $b$ through $a$ to $c$ at $-\infty$, and then from $c$ at $+\infty$ to $b$, the point $s$ describes the sides $B A, A C, C B$ of the shaded triangle marked $K$.

59. Suppose that the point $x$ is at $k$, in the shaded half-plane at an indefinitely small distance from $a$; say we have $x=-2 \kappa^{2} i$, ( $\kappa$ small), then taking for $\sqrt{x}$ the value $\kappa(1-i)$, we have $s^{3}=\frac{1-\kappa(1-i)}{1+\kappa(1-i)},=1-2 \kappa(1-i)$ nearly, and hence a value of $s$ is $=1-\frac{2}{3} \kappa+\frac{2}{3} \kappa i$, which belongs to a point $K$ near $A$, and within the shaded triangle: we have thus, in respect of this value of $s$, the shaded half of the $x$-plane corresponding to this shaded triangle. To the same value $x=-2 \kappa^{2} i$, correspond in all six values of $s$, giving six points $K$ each lying near a point $A$ within one of the shaded triangles; and hence the shaded half-plane corresponds to the six shaded triangles, and the unshaded half-plane corresponds to the six unshaded triangles.

60. Suppose the equation is

that is,

$$
\{s, x\}=\left(\mathrm{a}, \mathrm{b}, \mathrm{c} \therefore \gamma \frac{1}{x-a}, \frac{1}{x-b}, \frac{1}{x-c}\right)^{2},
$$

$$
=\frac{-(b-c)(c-a)(a-b)}{x-a \cdot x-b \cdot x-c}\left(\frac{a}{b-c \cdot x-a}+\frac{b}{c-a \cdot x-b}+\frac{c}{a-b \cdot x-c}\right),
$$

where $\mathrm{a}, \mathrm{b}, \mathrm{c}$ are real, but $a, b, c$ are imaginary. It is to be shown that, if the path of $x$ is the circle passing through the points $a, b, c$, then the path of $s$ is a circle passing through the corresponding three points.

61. We may find $\alpha, \beta, \gamma, \theta_{0}, \theta_{1}, \theta_{2}$, such that $a, b, c$ are $=\alpha+\beta i+\gamma e^{\theta_{0} i}, \alpha+\beta i+\gamma e^{\theta_{1} i}$, $\alpha+\beta i+\gamma e^{\theta_{2} i}$ (this is, in fact, finding $\alpha$ and $\beta$ the coordinates of the centre, and $\gamma$ the radius of the circle through the three points $a, b, c)$ : we then have $x=\alpha+\beta i+\gamma e^{\theta i}$, $\theta$ a variable parameter, the equation which expresses that the point $x$ is situate on the circle in question.

We have $x-a=\gamma\left(e^{\theta i}-e^{-\theta_{0} i}\right),=\gamma e^{\frac{1}{2}\left(\theta+\theta_{0}\right)}\left\{e^{\frac{1}{2}\left(\theta-\theta_{0}\right) i}-e^{-\frac{1}{2}\left(\theta-\theta_{0}\right) i}\right\}$; the second factor is $i \sin \frac{1}{2}\left(\theta-\theta_{0}\right),=i P$ suppose, or the equation is $x-a=i P \gamma e^{\frac{1}{2}\left(\theta+\theta_{0}\right) i}$, say

$$
x-a=i P_{\gamma} \operatorname{expi} \frac{1}{2}\left(\theta+\theta_{0}\right) \text {. }
$$

Similarly $x-b=i Q \gamma$ expi $\frac{1}{2}\left(\theta+\theta_{1}\right)$, and $x-c=i R \gamma$ expi $\frac{1}{2}\left(\theta+\theta_{2}\right)$; where $P, Q, R$ denote $\sin \frac{1}{2}\left(\theta-\theta_{0}\right), \sin \frac{1}{2}\left(\theta-\theta_{1}\right)$, $\sin \frac{1}{2}\left(\theta-\theta_{2}\right)$ respectively. In like manner, we have $b-c, c-a$, $a-b,=i F \gamma$ expi $\frac{1}{2}\left(\theta_{1}+\theta_{2}\right), i G \gamma$ expi $\frac{1}{2}\left(\theta_{2}+\theta_{0}\right)$, iHy expi $\frac{1}{2}\left(\theta_{0}+\theta_{1}\right)$, where $F, G, H$ denote $\sin \frac{1}{2}\left(\theta_{1}-\theta_{2}\right), \sin \frac{1}{2}\left(\theta_{2}-\theta_{0}\right), \sin \frac{1}{2}\left(\theta_{0}-\theta_{1}\right)$ respectively. 
We have

$$
\begin{array}{r}
-\frac{b-c \cdot c-a \cdot a-b}{x-a \cdot x-b \cdot x-c}=\frac{-F G H}{P Q R} \operatorname{expi} \frac{1}{2}\left(\theta_{0}+\theta_{1}+\theta_{2}-3 \theta\right) \\
\frac{1}{b-c \cdot x-a}=\frac{-1}{\gamma^{2} P F} \text { expi }-\frac{1}{2}\left(\theta_{0}+\theta_{1}+\theta_{2}+\theta\right)
\end{array}
$$

with the like values for $\frac{1}{c-a \cdot x-b}$ and $\frac{1}{a-b \cdot x-c}$. Hence the right-hand side of the equation is

$$
=\frac{F G H}{P Q R}\left(\frac{\mathrm{a}}{P F}+\frac{\mathrm{b}}{Q G}+\frac{\mathrm{c}}{R H}\right) \operatorname{expi}(-2 \theta)
$$

62. Considering now the left-hand side of the equation, we have

$$
\{s, x\}=\frac{1}{\left(\frac{d x}{d \theta}\right)^{2}}(\{s, \theta\}-\{x, \theta\}) ;
$$

substituting for $x$ its value $=\alpha+\beta i+\gamma e^{\theta i}$, this becomes

that is,

$$
\{s, x\}=-\frac{1}{\gamma^{2}} e^{-2 \theta i}\left(\{s, \theta\}-\frac{1}{2}\right),
$$

$$
=-\frac{1}{\gamma^{2}}\left(\{s, \theta\}-\frac{1}{2}\right) \operatorname{expi}(-2 \theta) \text {. }
$$

Assume $s=L+M i+N e^{\ominus i}, L, M$, and $N$ constants; then using the accent to denote differentiation in regard to $\theta$, we find without difficulty $\{s, \theta\}=\{\Theta, \theta\}+\frac{1}{2} \Theta^{\prime 2}$, and the value of $\{s, x\}$ becomes

$$
=-\frac{1}{\gamma^{2}}\left(\{\Theta, \theta\}+\frac{1}{2} \Theta^{\prime 2}-\frac{1}{2}\right) \operatorname{expi}(-2 \theta) .
$$

Hence, substituting the values of the two sides of the equation, the imaginary factor expi $(-2 \theta)$ divides out, and the equation becomes

$$
\{\Theta, \theta\}+\frac{1}{2} \Theta^{\prime 2}-\frac{1}{2}=-\frac{F G H}{P Q R}\left(\frac{\mathrm{a}}{P F}+\frac{\mathrm{b}}{Q G}+\frac{\mathrm{c}}{R H}\right),
$$

an equation, in which everything is real and which thus determines $\Theta$ as a real function of $\theta$ : and we have therefore the theorem in question.

Connexion with the differential equation for the hypergeometric series. Art. Nos. 63 to 68 .

63. Take $p, q$ given functions of $x$, and $y$ a function of $x$ determined by the equation

$$
\frac{d^{2} y}{d x^{2}}+p \frac{d y}{d x}+q y=0
$$


again $P, Q$ given functions of $z$, and $v$ a function of $z$ determined by the equation

and assume

$$
\frac{d^{2} v}{d z^{2}}+P \frac{d v}{d z}+Q v=0
$$

$$
y=w v .
$$

Substituting this value of $y$ in the first equation, we obtain for $v$ an equation of the second order (the coefficients of which contain $w$ ), and we may make this identical with the second equation; viz. comparing the coefficients of the two equations, we thus have two equations each containing $w$; and by eliminating $w$ we obtain a differential equation of the third order between $z$ and $x$. This is, in fact, the basis of Kummer's theory for the transformation of a hypergeometric series: the equation between $z, x$ will be found presently in a different manner.

64. But if with Schwarz, instead of making the equation obtained for $v$ as above identical with the given equation for $v$, we merely assume that the two equations are consistent, then there is nothing to-determine the value of $z$, which may be regarded as an arbitrary function of $x ; y$ and $v$ are then functions of $x$, and $w$ denotes the quotient $y \div v$ of these two functions, and as such satisfies an equation the form of which will depend on the assumed relation between $z$ and $x$. In particular, if $P$ and $Q$ denote the same functions of $z$ that $p$ and $q$ are of $x$; and if we assume $z=x$, $P, Q$ will become $=p, q$ respectively: the given equation in $v$ will be

$$
\frac{d^{2} v}{d x^{2}}+p \frac{d v}{d x}+q v=0 ;
$$

and $w$ will thus denote the quotient of any two solutions of the equation

$$
\frac{d^{2} y}{d x^{2}}+p \frac{d y}{d x}+q y=0 ;
$$

viz. writing $X=p^{2}+2 \frac{d p}{d x}-4 q$, then, by what precedes, the equation for $w$ will be

$$
\{w, x\}=-\frac{1}{2} X \text {. }
$$

65. Returning now to Kummer's problem, and considering $y, v$ as solutions of the two differential equations respectively, $w$ is a function independent of the particular solutions denoted by these letters: we have $y=w v$, and taking any other two solutions we have $y_{1}=w v_{1}$, so that $\frac{y}{y_{1}}=\frac{v}{v_{1}}$; calling each of these equal quantities $s$, we have $s$ denoting the quotient of two solutions of the equation in $y$, and also the quotient of two solutions of the equation in $v$; whence, writing as before $X=p^{2}+2 \frac{d p}{d x}-4 q$, and similarly $Z=P^{2}+2 \frac{d P}{d z}-4 Q$, we have

and since in general

$$
\{s, x\}=-\frac{1}{2} X, \quad\{s, z\}=-\frac{1}{2} Z,
$$

$$
\{s, x\}=\left(\frac{d z}{d x}\right)^{2}\{s, z\}+\{z, x\},
$$

C. XI. 
we obtain

$$
\{z, x\}=-\frac{1}{2} X+\frac{1}{2} Z\left(\frac{d z}{d x}\right)^{2}
$$

as the required equation for the determination of $z$ as a function of $x$. The process does not give the value of $w$, but this can be found without difficulty, viz.

$$
w^{2}=C e^{\int P d z-j p d x} \div \frac{d z}{d x}
$$

If $z, x$ are regarded each of them as a function of the new independent variable $\theta$, then the equation is

$$
\{z, \theta\}-\frac{1}{2}\left(\frac{d z}{d \theta}\right)^{2} Z=\{x, \theta\}-\frac{1}{2}\left(\frac{d x}{d \theta}\right)^{2} X
$$

66. Jacobi's differential equation of the third order for the transformed modulus $\lambda$, Fund. Nova, p. 78, [Ges. Werke, t. I, p. 132], is

$$
3\left(k^{\prime 2} \lambda^{\prime \prime 2}-\lambda^{\prime 2} k^{\prime \prime 2}\right)-2 k^{\prime} \lambda^{\prime}\left(k^{\prime} \lambda^{\prime \prime \prime}-\lambda^{\prime} k^{\prime \prime \prime}\right)+k^{\prime 2} \lambda^{\prime 2}\left\{\left(\frac{1+k^{2}}{k-k^{3}}\right)^{2} k^{\prime 2}-\left(\frac{1+\lambda^{2}}{\lambda-\lambda^{3}}\right)^{2} \lambda^{\prime 2}\right\}=0,
$$

where the accents denote differentiations in regard to an independent variable $\theta$ : viz. dividing by $2 k^{\prime 2} \lambda^{\prime 2}$, this becomes

$$
\{k, \theta\}+\frac{1}{2} k^{\prime 2}\left(\frac{1+k^{2}}{k-k^{3}}\right)^{2}=\{\lambda, \theta\}+\frac{1}{2} \lambda^{\prime 2}\left(\frac{1+\lambda^{2}}{\lambda-\lambda^{3}}\right)^{3},
$$

which is thus a particular case of Kummer's equation, $k, \lambda$ corresponding to $x, z$ respectively, and the values of $X, Z$ being

$$
X=-\left(\frac{1+k^{2}}{k-k^{3}}\right)^{2}, Z=-\left(\frac{1+\lambda^{2}}{\lambda-\lambda^{3}}\right)^{3}
$$

67. In the case of the hypergeometric series, the two differential equations of the second order are

$$
\begin{aligned}
& \frac{d^{2} y}{d x^{2}}+\frac{\gamma-(\alpha+\beta+1) x}{x \cdot 1-x} \frac{d y}{d x}-\frac{\alpha \beta y}{x \cdot 1-x}=0 \\
& \frac{d^{2} v}{d z^{2}}+\frac{\gamma^{\prime}-\left(\alpha^{\prime}+\beta^{\prime}+1\right) z}{z \cdot 1-z} \frac{d v}{d z}-\frac{\alpha^{\prime} \beta^{\prime} v}{z \cdot 1-z}=0 .
\end{aligned}
$$

Hence

$$
p=\frac{\gamma(x+(1-x))-(\alpha+\beta+1) x}{x \cdot 1-x}=\frac{\gamma}{x}+\frac{\gamma-\alpha-\beta-1}{1-x}, q=\frac{-\alpha \beta}{x .1-x},
$$

and hence

$$
p^{2}+2 \frac{d p}{d x}-4 q=\frac{\gamma^{2}-2 \gamma}{x^{2}}+\frac{(\gamma-\alpha-\beta-1)^{2}+2(\gamma-\alpha-\beta-1)}{(1-x)^{2}}+\frac{4 \alpha \beta+2 \gamma(\gamma-\alpha-\beta-1)}{x .1-x}
$$

viz. writing

$$
\begin{array}{ll}
\lambda^{2}=(1-\gamma)^{2}, & \mathrm{a}=\frac{1}{2}\left(1-\lambda^{2}\right), \\
\mu^{2}=(\alpha-\beta)^{2}, & \mathrm{~b}=\frac{1}{2}\left(1-\mu^{2}\right), \\
\nu^{2}=(\gamma-\alpha-\beta)^{2}, & \mathrm{c}=\frac{1}{2}\left(1-\nu^{2}\right),
\end{array}
$$


and putting in the formula $x-1,=-(1-x)$, we have

$$
\begin{aligned}
-\frac{1}{2}\left(p^{2}+2 \frac{d p}{d x}-4 q\right) & =\frac{\frac{1}{2}\left(1-\lambda^{2}\right)}{x^{2}}+\frac{\frac{1}{2}\left(1-\nu^{2}\right)}{(x-1)^{2}}+\frac{\frac{1}{2}\left(\lambda^{2}-\mu^{2}+\nu^{2}-1\right)}{x . x-1} \\
& =\frac{\mathrm{a}}{x^{2}}+\frac{\mathrm{c}}{(x-1)^{2}}+\frac{-\mathrm{a}+\mathrm{b}-\mathrm{c}}{x \cdot x-1} \\
& =\left(\mathrm{a}, \mathrm{b}, \mathrm{c} \therefore\left(\frac{1}{x}, \frac{1}{x-\infty}, \frac{1}{x-1}\right)^{2}\right.
\end{aligned}
$$

with a like formula for $\frac{1}{2}\left(P^{2}+2 \frac{d P}{d z}-4 Q\right)$. We then have

$$
\begin{aligned}
y & =w v \\
w^{2} & =C x^{-\gamma}(1-x)^{\gamma-\alpha-\beta-1} z^{\gamma^{\prime}}(1-z)^{-\gamma^{\prime}+a^{\prime}+\beta^{\prime}+1} \frac{d x}{d z},
\end{aligned}
$$

and the differential equation of the third order for the determination of $z$ is

$$
\{z, x\}+\left(\mathrm{a}_{1}, \mathrm{~b}_{1}, \mathrm{c}_{1} \therefore \chi \frac{1}{z}, \frac{1}{z-\infty}, \frac{1}{z-1}\right)^{2}\left(\frac{d z}{d x}\right)^{2}-\left(\mathrm{a}, \mathrm{b}, \mathrm{c} \therefore \chi \frac{1}{x}, \frac{1}{x-\infty}, \frac{1}{x-1}\right)^{2}=0 \text {, }
$$

where $a_{1}, b_{1}, c_{1}$ are the same functions of $\alpha^{\prime}, \beta^{\prime}, \gamma^{\prime}$ which $a, b, c$ are of $\alpha, \beta, \gamma$. This is, in effect, Kummer's equation for the transformation of the hypergeometric series.

68. And in like manner the Schwarzian equation for the determination of $s$, the quotient of two solutions, is

$$
\{s, x\}=\left(\mathrm{a}, \mathrm{b}, \mathrm{c} \therefore \chi_{\chi} \frac{1}{x}, \frac{1}{x-\infty}, \frac{1}{x-1}\right)^{2} .
$$

\section{PART II. THE POLYHEDRAL FUNCTIONS.}

Origin and Properties. Art. Nos. 69 to 80.

69. The functions in lines $1, \ldots, 5$ of the $P Q R$-Table are connected with the geometrical forms:

$$
\begin{aligned}
& \left\{\begin{array}{l}
\text { 1. } \\
\text { 2. }
\end{array}\right. \text { Double Pyramid *, } \\
& \text { 3. Tetrahedron, } \\
& \text { 4. Octahedron and Cube, } \\
& \text { 5. Dodecahedron and Icosahedron, }
\end{aligned}
$$

(these figures being regarded as situate on a spherical surface), and with the stereographic projections of these figures.

* Prof. Klein regards 1 as belonging to the polygon and 2 to the double pyramid: it seems to me that the fundamental figure, to which 1 and 2 each of them belong, is the polygon. 
Consider a spherical surface and upon it any number of points: take at pleasure any point as South Pole, this determines the plane of the equator; and the stereographic projection of any point is the intersection with the plane of the equator of the line joining the point with the South Pole.

To fix the ideas take the radius of the sphere as unity: let the axes of $x$ and $y$ be drawn in the plane of the equator in longitudes $0^{\circ}$ and $90^{\circ}$ respectively, and the axis of $z$ upwards through the North Pole: the position of a point on the sphere is determined by means of its N.P.D. $\theta$ and longitude $f$ : moreover we take $X, Y, Z$ for the coordinates of the point on the surface, and $x, y$ for those of its projection; and we then have

$$
\begin{gathered}
X, Y, Z=\sin \theta \cos f, \sin \theta \sin f, \cos \theta ; \\
x=\frac{X}{1+Z}=\tan \frac{1}{2} \theta \cos f, \quad y=\frac{Y}{1+Z}=\tan \frac{1}{2} \theta \sin f,
\end{gathered}
$$

and conversely,

$$
X, Y, Z=2 x, 2 y, 1-x^{2}-y^{2}, \div\left(1+x^{2}+y^{2}\right) .
$$

We represent the point $(X, Y, Z)$ on the spherical surface by means of the magnitude $x+i y,=\tan \frac{1}{2} \theta(\cos f+i \sin f)$, or say by the linear factor, $s-(x+i y)$ : and similarly any system of points on the surface by means of the system of magnitudes $x+i y$, or say by the function $\Pi\{s-(x+i y)\}$, denoting in this manner the product of the linear factors which correspond to the different points respectively.

70. It will presently appear that, if (considering a different stereographic projection, that is, a different position of the South Pole) we take $x^{\prime}, y^{\prime}$ as the coordinates of the new projection of the point, then $x^{\prime}+i y^{\prime}$ is a homographic function

$$
a(x+i y)+b \div\{c(x+i y)+d\}
$$

of $x+i y$ : and consequently that the functions of $s$, which belong to different projections, are linear transformations one of the other: but at present we consider a single projection.

It may be proper to remark that the figures in question are spherical figures having summits which are points on the spherical surface, edges (or sides) which are arcs of great circle joining two summits, and faces which are portions of the spherical surface: the mid-points of the sides, and the centres of the faces are of course points on the spherical surface.

71. (1), (2). Considering a regular polygon formed by $n$ summits on the equator, the longitude of one of them being $0^{\circ}$, then the stereographic projections correspond with the points themselves, and the values of $x+i y$ are

$$
1, \cos \frac{2 \pi}{n}+i \sin \frac{2 \pi}{n}, \ldots, \cos \frac{(n-1) 2 \pi}{n}+i \sin \frac{(n-1) 2 \pi}{n} .
$$

The corresponding function of $s$ is $s^{n}-1$. 
The values of $x+i y$ for the mid-points of the sides are

$$
\cos \frac{\pi}{n}+i \sin \frac{\pi}{n}, \cos \frac{3 \pi}{n}+i \sin \frac{3 \pi}{n}, \ldots, \cos \frac{(2 n-1) \pi}{n}+i \sin \frac{(2 n-1) \pi}{n} .
$$

The corresponding function of $s$ is $s^{n}+1$.

The North and South Poles, which form with the $n$ points a double pyramid of $n+2$ summits, correspond to the values $s=0$ and $s=\infty$. We have thus

$$
s\left(1-\frac{s}{\infty}\right)\left(s^{n}-1\right)
$$

as the function corresponding to the double pyramid.

72. (3). Considering for a moment the tetrahedron as a figure with rectilinear edges, this is so placed that two opposite edges are horizontal, and that the vertical planes passing through the centre and these two edges respectively are inclined at angles $\pm 45^{\circ}$ to the meridian: viz. the upper edge has the longitudes $135^{\circ}, 315^{\circ}$, and the lower edge the longitudes $45^{\circ}, 225^{\circ}$. We thus explain the position of the spherical figure.

Corresponding to the summits we have the function $s^{4}-2 i \sqrt{ } 3 s^{2}+1$.

In fact, the equation $s^{4}-2 i \sqrt{ } 3 s^{2}+1=0$ gives $s^{2}=i(\sqrt{ } 3 \pm 2)$, and hence the values of $s$ are the four values of $x+i y$ shown in the annexed table for the values of $X, Y, Z$, and $x+i y$ for the summits of the tetrahedron,

$$
\begin{aligned}
& \begin{array}{lrr|r}
\text { long. } X & Y & Z & x+i y \\
\hline 45^{\circ} \frac{1}{\sqrt{ } 3} & \frac{1}{\sqrt{ } 3} & -\frac{1}{\sqrt{ } 3} & \frac{1+i}{\sqrt{ } 3-1}
\end{array} \\
& 135^{\circ}-+\quad+\frac{-1+i}{\sqrt{ } 3+1} \\
& 225^{\circ}-\quad-\quad-\frac{-1-i}{\sqrt{ } 3-1} \\
& 315^{\circ}+-\quad+\frac{1+i}{\sqrt{ } 3+1} .
\end{aligned}
$$

Corresponding to the centres of the faces, or summits of the opposite tetrahedron, we have the function $s^{4}+2 i \sqrt{ } 3 s^{2}+1$.

Corresponding to the mid-points of the sides, we have the function

$$
s\left(1-\frac{s}{\infty}\right)\left(s^{4}-1\right) \text {; }
$$

viz. the points in question are the North Pole $s=0$, the South Pole $s=\infty$, and the four points $s= \pm 1, s= \pm i$ on the equator at longitudes $0^{\circ}, 90^{\circ}, 180^{\circ}, 270^{\circ}$ respectively. 
73. (4). The octahedron is placed with two of its summits as poles, and the other four summits in the equator at longitudes $0^{\circ}, 90^{\circ}, 180^{\circ}, 270^{\circ}$ respectively: the values of $s$ are, as in the last case, $0, \infty, \pm 1, \pm i$, and the function is

$$
s\left(1-\frac{s}{\infty}\right)\left(s^{4}-1\right) \text {. }
$$

The function for the centres of the faces, or summits of the cube, is $s^{8}+14 s^{4}+1$.

The function for the mid-points of the sides of the octahedron or of the cube is

$$
s^{12}-33 s^{8}-33 s^{4}+1 \text {. }
$$

74. (5). The Icosahedron is placed with two of its summits for poles; five summits lying in a small circle above the plane of the equator at longitudes $0^{\circ}, 72^{\circ}, 144^{\circ}, 288^{\circ}$, and the remaining five summits in the corresponding small circle below the equator at longitudes $36^{\circ}, 108^{\circ}, 180^{\circ}, 252^{\circ}$ and $324^{\circ}$.

The function for the summits of the Icosahedron is

$$
s\left(1-\frac{s}{\infty}\right)\left(s^{10}+11 s^{5}-1\right) .
$$

The function for the centres of the faces of the Icosahedron, or summits of the Dodecahedron, is $s^{20}-228 s^{15}+494 s^{10}+228 s^{5}-1$. is

The function for the mid-points of the sides of the Icosahedron or the Dodecahedron

$$
s^{30}-522 s^{25}+10005 s^{20}+0 s^{15}-10005 s^{10}+522 s^{5}+1 .
$$

I give for the present these results without demonstration.

75. Writing $\frac{x}{y}$ for $s$ so as to obtain homogeneous functions $\left(* \nmid(x, y)^{n}\right.$,-it will be recollected that the $x, y$ of these functions have nothing to do with the $x, y$ of the foregoing values $x+i y$-the forms which have thus presented themselves may be denoted as follows :

$$
\begin{aligned}
(3): \quad f 3 & =\left(1,-2 i \sqrt{ } 3,1 \chi x^{2}, y^{2}\right)^{2}, \\
h 3 & =\left(1,+2 i \sqrt{ } 3,1 \gamma\left(x^{2}, y^{2}\right)^{2},\right. \\
t 3 & =x y\left(x^{4}-y^{4}\right), \\
(4): \quad f 4 & =x y\left(x^{4}-y^{4}\right), \\
h 4 & =\left(1,14,1 \gamma\left(x^{4}, y^{4}\right)^{2},\right. \\
t 4 & =\left(1,-33,-33,1 \gamma\left(x^{4}, y^{4}\right)^{3},\right. \\
(5): \quad f 5 & =x y\left(1,11,-1 \gamma\left(x^{5}, y^{5}\right)^{2},\right. \\
h 5 & =\left(1,-228,+494,+228,-1 \gamma\left(x^{5}, y^{5}\right)^{4},\right. \\
t 5 & =\left(1,-522,10005,0,-10005,522,1 \gamma x^{5}, y^{5}\right)^{6},
\end{aligned}
$$

where observe that $f 4$ is the same function as $t 3$. In each set of functions $f, h, t$, we have $h$ and $t$ covariants of $f$, viz. disregarding numerical factors,

$h$ is the Hessian, or derivative $(f, f)^{2}$, and $t$ is the derivative $(f, h)$. 
76. Since $f 4$ is the same function as $t 3$, we have of course $f 4, h 4$ and $t 4$ themselves covariants of $f 3:$ but it is convenient to separate the two systems.

77. It is to be observed that $f 3$ is a quartic function having its quadrinvariant $(I)=0$; but independently of this, that is, quà quartic function, it has only the covariants $h 3$ and $t 3$ (the Hessian and the cubicovariant respectively), viz. every other covariant is a rational and integral function of $f 3, h 3$ and $t 3$. In particular, $h 4$ and $t 4$ are rational and integral functions of $f 3, h 3$ and $t 3$; but inasmuch as $f 3$ and $h 3$ are not covariants of $f 4$, this is not a property of $h 4$ and $t 4$ considered as covariants of $f 4$, and the relation in question need not be attended to.

78. It has just been stated that $f 3$ quà quartic function has (in the sense explained) only the covariants $h 3$ and $t 3: f 4$ quà special sextic function and $f 5$ quà special dodecadic function have the like property, viz. $f 4$ has only the covariants $h 4$ and $t 4 ; f 5$ only the covariants $h 5$ and $t 5$. Hence $f 3, f 4, f 5$ are "Prime-forms" in the sense defined in the paper by Fuchs, of 1875, viz. a Prime-form has no covariant of a lower order than itself, and also no covariant of a higher order which is a power of a form of a lower order.

79. The same functions have also the property that they are functions transformable into themselves by means of a group of linear transformations, and in this point of view they were considered in the nearly contemporaneous paper by Klein, of 1875 ; it is in this paper shown that the functions so transformable into themselves must be Polyhedral functions as above, the linear transformations in fact corresponding to the rotations whereby the spherical polyhedron can be brought into coincidence with its own original position. This theory will be presently given.

80. It is to be observed that, if $U, V$ are functions $\left(* \gamma(x, y)^{n}\right.$ of the same order $n$, then using the accent to denote differentiation in regard to $x, U V^{\prime}-U^{\prime} V$ and $(U, V)$ differ only by a numerical factor: and further that, writing as before $s=\frac{x}{y}$, and in the expression $U V^{\prime}-U^{\prime} V$ regarding $U, V$ as functions $\left(* \gamma(s, 1)^{n}\right.$, and the accent as denoting differentiation in regard to $s$, we have $U V^{\prime}-U^{\prime} V$ and $(U, V)$ differing by a numerical factor only. We have in the $P Q R$-Table, lines $3,4,5$, $P, Q, R$ equal to given numerical multiples of $h^{\beta}, t^{\gamma}, f^{\alpha}$, the indices $\alpha, \beta, \gamma$ being such as to make these to be functions of the same degree: hence, neglecting numerical multipliers, $P Q^{\prime}-P^{\prime} Q$ is equal to a function $\left(h^{\beta}, t^{\gamma}\right)$, which is $=h^{\beta-1} t^{\gamma-1}(h, t)$ : and the theorem that $P Q^{\prime}-P^{\prime} Q,=Q R^{\prime}-Q^{\prime} R,=R P^{\prime}-R^{\prime} P$, contains only factors of $P, Q, R$ is in fact the theorem that $(h, t),(h, f)$, and $(t, f)$ are each of them equal to a term or product of $f, h, t$ : which is a result included in the theorem that $f$ has only the covariants $h$ and $t$. And by this last theorem we know already how from $R$, assumed to be known, we can derive $P$ and $Q$ : viz. $R$ is a power of $f$; and we thence have $h=(f, f)^{2}$ and $t=(h, f)$, equations giving the functions $h$ and $t$, upon which $P$ and $Q$ depend. 
Covariantive Formulce. Art. Nos. 81 to 84 .

81. The various covariantive formulæ will be given with their proper numerical coefficients.

Tetrahedron function. $f, h, t$ stand for the before-mentioned values,

For $f 3$.

$$
f 3, h 3, t 3\left(P, Q, R=h^{3},-12 i \sqrt{ } 3 \cdot t^{2},-f^{3}\right) \text {. }
$$

$$
\begin{aligned}
&(a, b, c, d, e)=1,0, \frac{-i}{\sqrt{ } 3}, 0,1 . \\
& \frac{1}{2}(f, f)^{2}=-96 i \sqrt{ } 3 \cdot h, \quad \frac{1}{2}(h, h)^{2}=96 i \sqrt{ } 3 \cdot f, \quad \frac{1}{2}(t, t)^{2}=-25 f h, \\
&(f, h)=32 i \sqrt{ } 3 \cdot t, \quad(f, f)^{4}=576 I=0, \quad(f, h)^{4}=1152 J= \\
&(f, t)=\quad 4 \cdot h^{2}, \\
&(h, t)=\quad 4 \cdot f^{2}, \\
& h^{3}-f^{3}=12 i \sqrt{ } 3 t^{2}=0, \\
& f h=\left(1,14,1 \gamma\left(x^{4}, y^{4}\right)^{2}(=f 4) .\right.
\end{aligned}
$$

It is convenient to remark that $t^{2}, f^{3}, h^{3}$ being of the same order we have

that is,

$$
t^{2}\left(f^{3}, h^{3}\right)+f^{3}\left(h^{3}, t^{2}\right)+h^{3}\left(t^{2}, f^{3}\right)=0,
$$

$$
t^{2} \cdot 3 \cdot 3 f^{2} h^{2}(f, h)+f^{3} \cdot 3 \cdot 2 h^{2} t(h, t)+h^{3} \cdot 2 \cdot 3 t f^{2}(t, f)=0,
$$

an equation which, substituting for $(f, h),(h, t),(t, f)$ their values, reduces itself to the before-mentioned relation $h^{3}-f^{3}-12 i \sqrt{ } 3 t^{2}=0$; and we have thus a verification of the values of $(f, h),(h, t)$ and $(t, f)$. The like remark applies to the other two cases, which follow.

82. Hexahedron function. $f, h, t$ stand for the before-mentioned values

For $f 4$.

$$
f 4, h 4, t 4\left(P, Q, R=h^{3},-t^{2},-108 f^{4}\right) \text {. }
$$

$$
\begin{aligned}
& (a, b, c, d, e, f, g)=\left(0, \frac{1}{4}, 0,0,0,-\frac{1}{4}, 0\right) . \\
& \frac{1}{2}(f, f)^{2}=-25 h, \quad \frac{1}{2}(f, f)^{4}=0, \quad \frac{1}{2}(f, f)^{6}=(720)^{2} \cdot \frac{3}{8}, \\
& (f, h)=-8 t, \quad \frac{1}{2}(h, h)^{2}=3 \cdot 2^{6} \cdot 7^{2} \cdot f^{2}, \\
& (f, t)=-12 h^{2}, \quad \frac{1}{2}(t, t)^{2}=2^{4} \cdot 3^{3} \cdot 11^{2} \cdot f^{2} h, \\
& (h, t)=-1728 f^{3}, \\
& h^{3}-t^{2}-108 f^{4}=0 \text {. }
\end{aligned}
$$


83. Dodecahedron function. $f, h, t$ stand for the before-mentioned values

For $f 5$.

$$
f 5, h 5, t 5\left(P, Q, R=h^{3},-t^{2},-1728 f^{5}\right) \text {. }
$$

$$
\begin{array}{lll}
(a, b, c, d, e, f, g, h, i, j, k, l, m) & =\left(0, \frac{1}{12}, 0,0,0,0, \frac{1}{84}, 0,0,0,0,-\frac{1}{12}, 0\right) . \\
\frac{1}{2}(f, f)^{2}=-121 h, & \frac{1}{2}(f, f)^{4}=0, & \frac{1}{2}(f, f)^{6}=\frac{1}{2}(924)^{2}(720)^{2} \cdot \frac{5}{84} f^{*}, \\
\frac{1}{2}(f, f)^{8}=0, & \frac{1}{2}(f, f)^{10}=0, & \frac{1}{2}(f, f)^{12}=\frac{1}{2}(924)^{2}(720)^{4} \cdot \frac{25}{84} * \\
(f, h)=-20 t, & \frac{1}{2}(h, h)^{2}=173280 f^{3}, \\
(f, t)=-30 h^{2}, & \frac{1}{2}(t, t)^{2}=9082800 f^{3} h, \\
(h, t)=-86400 f^{5}, & &
\end{array}
$$

84. We have

Write

$$
t=\left(x^{10}+y^{10}\right)\left(1,522,-10006,-522,1 \gamma\left(x^{5}, y^{5}\right)^{4} .\right.
$$

then

$$
\xi=\left(x^{2}+y^{2}\right) \cdot(1,2,6,-2,1 \gamma x, y)^{4},
$$

Or putting

$$
t=\xi\left(1,-10,45 \gamma \xi^{2}, f\right) \text {. }
$$

that is, $\xi=p \sqrt{ } f$, then

$$
p=\frac{\xi}{\sqrt{ } f}, \quad=\frac{\left(x^{2}+y^{2}\right)(1,2,6,-2,1 \gamma x, y)^{4}}{\sqrt{x y\left(x^{10}+11 x^{5} y^{5}-y^{10}\right)}},
$$

$$
\left.p^{5}-10 p^{3}+45 p=\frac{t}{\sqrt{ } f^{5}} . \quad \text { (Klein. }\right)
$$

Investigation of the forms $f 5$ and $h 5$. Art. Nos. 85 and 86 .

85. Writing for shortness $\dagger k=\tan \alpha=\frac{\sqrt{ } 5-1}{2}$, and $g=\cos 36^{\circ}+i \sin 36^{\circ}$, then the values of $x+i y$ corresponding to the summits of the Icosahedron are

$$
\begin{array}{lllll}
0, & & & \\
k, & k g^{2}, & k g^{4}, & k g^{6}, & k g^{8}, \\
k^{-1} g, & k^{-1} g^{3}, & k^{-1} g^{5}, & k^{-1} g^{7}, & k^{-1} g^{9}, \\
\infty ; & & & &
\end{array}
$$

and the function $f 5$ is thus

$$
=s\left(1-\frac{s}{\infty}\right)\left(s^{5}-k^{5}\right)\left(s-k^{-5}\right),
$$

* The numerical coefficients $-\frac{5}{84}$ and $\frac{25}{84}$ are Klein's $B$ and $A$ : the latter of them is the ordinary quadrinvariant of a dodecadic function; the former is an invariant linear as regards the coefficients of $f$, and existing only for the special form $f$ in question: viz. writing for a moment

$$
f=\lambda\left(x^{11} y+11 x^{6} y^{6}-x y^{11}\right),
$$

then $(f, f)^{6}$ contains the factor $\lambda^{2}$, and ( $f$ containing the factor $\lambda$ ) the form is

$$
\frac{1}{2}(f, f)^{6}=\frac{1}{2}(924)^{2}(720)^{2} \cdot-\frac{5}{84} \lambda \cdot f,
$$

which is linear as regards $\lambda$. We have also

$$
\frac{1}{2}(f, f)^{12}=\frac{1}{2}(924)^{2}(720)^{4} \cdot \frac{25}{84} \lambda^{2}:
$$

say $A=\frac{25}{84} \lambda^{2}, B=-\frac{5}{84} \lambda$; or $84 B^{2}=A$. Of course in the case of a general dodecadic function $f$, we have $(f, f)^{6}$, an irreducible covariant, not breaking up into factors.

$+a$ is the $a, \gamma$ is the $\gamma$, and $\gamma^{\prime}$ the $a-\beta$ of the Table, No. 99 .

C. XI. 
where the product of the last two factors is $s^{10}+\left(k^{-5}-k^{5}\right) s^{5}-1$. We have

$$
\begin{array}{ll}
k^{-5}=\frac{1}{32}(80 \sqrt{ } 5+176), & =\frac{1}{2}(5 \sqrt{ } 5+11), \\
k^{5}=\frac{1}{32}(80 \sqrt{ } 5-176), & =\frac{1}{2}(5 \sqrt{ } 5-11),
\end{array}
$$

and consequently $k^{-5}-k^{5}=11$; or the function is

$$
s\left(1-\frac{s}{\infty}\right)\left(s^{10}+11 s^{5}-1\right) .
$$

86. Similarly, writing for shortness* $l=\tan \frac{1}{2} \gamma, l^{\prime}=\tan \frac{1}{2} \gamma^{\prime}$, where

$$
\begin{array}{lll}
\cos ^{2} \gamma=\frac{5+2 \sqrt{ } 5}{15}, & \sin ^{2} \gamma=\frac{10-2 \sqrt{ } 5}{15} ; & \text { and therefore } \frac{\cos \gamma}{\sin \gamma}=\frac{3+\sqrt{ } 5}{4} ; \\
\cos ^{2} \gamma^{\prime}=\frac{5-2 \sqrt{ } 5}{15}, & \sin ^{2} \gamma^{\prime}=\frac{10+2 \sqrt{ } 5}{15} ; & \quad, \quad \frac{\cos \gamma^{\prime}}{\sin \gamma^{\prime}}=\frac{3-\sqrt{ } 5}{4} ;
\end{array}
$$

and $g=\cos 36^{\circ}+i \sin 36^{\circ}$ as before, then the values of $x+i y$ for the summits of the dodecahedron are

$$
\begin{array}{lllll}
l g, & l g^{3}, & l g^{5}, & l g^{7}, & l g^{9}, \\
l^{\prime} g, & l^{\prime} g^{3}, & l^{\prime} g^{5}, & l^{\prime} g^{7}, & l^{\prime} g^{9}, \\
l^{\prime-1}, & l^{\prime-1} g^{2}, & l^{\prime-1} g^{4}, & l^{\prime-1} g^{6}, & l^{\prime-1} g^{8}, \\
l^{-1}, & l^{-1} g^{2}, & l^{-1} g^{4}, & l^{-1} g^{6}, & l^{-1} g^{8} .
\end{array}
$$

The function $h 5$ is therefore

We have

$$
=s^{10}+s^{5}\left(l^{5}-l^{-5}\right)+1 . s^{10}+s^{5}\left(l^{\prime 5}-l^{\prime-5}\right)-1 .
$$

$$
\begin{aligned}
l^{-5}-l^{5} & =\frac{(1+\cos \gamma)^{5}-(1-\cos \gamma)^{5}}{\sin ^{5} \gamma}=\frac{2 \cos \gamma}{\sin ^{5} \gamma}\left(5+10 \cos ^{2} \gamma+\cos ^{4} \gamma\right) \\
& =\frac{2 \cos \gamma}{\sin ^{5} \gamma} \cdot \frac{384+64 \sqrt{ } 5}{45}=\frac{128}{45} \frac{\cos \gamma}{\sin ^{5} \gamma}(6+\sqrt{ } 5)=114+50 \sqrt{ } 5
\end{aligned}
$$

viz. this last identity depends on

that is,

$$
\frac{32}{45}(3+\sqrt{ } 5)(6+\sqrt{ } 5)=(114+50 \sqrt{ } 5) \sin ^{4} \gamma
$$

or

$$
160(3+\sqrt{ } 5)(6+\sqrt{ } 5)=(114+50 \sqrt{ } 5)(120-40 \sqrt{ } 5),
$$

or finally

$$
2(3+\sqrt{ } 5)(6+\sqrt{ } 5)=(57+25 \sqrt{ } 5)(3-\sqrt{ } 5),
$$

which is right.

$$
(7+3 \sqrt{ } 5)(6+\sqrt{ } 5)=57+25 \sqrt{ } 5,
$$

Similarly

$$
l^{\prime-5}-l^{\prime 5}=114-50 \sqrt{ } 5,
$$

and observing that the sum and product of $114+50 \sqrt{ } 5,114-50 \sqrt{ } 5$ are $=228$ and 496 respectively, the required function of $s$ is

$$
\begin{aligned}
& \left(s^{10}-1\right)^{2}-228\left(s^{15}-s^{5}\right)+496 s^{10}, \\
= & s^{20}-228 s^{15}+494 s^{10}+228 s^{5}+1,
\end{aligned}
$$

which is the required value of $h 5$. 
Invariantive property of the Stereographic Projection. Art. Nos. 87 to 93 .

87. The before-mentioned theorem that the functions derived from two different stereographic projections of the same point are linear transformations one of the other, may be thus stated:

Considering on the surface of a sphere, two fixed points $A$ and $B$; and determining the position of a point $C$, first in regard to $A$ by its distance $\theta$ and azimuth $f$, and

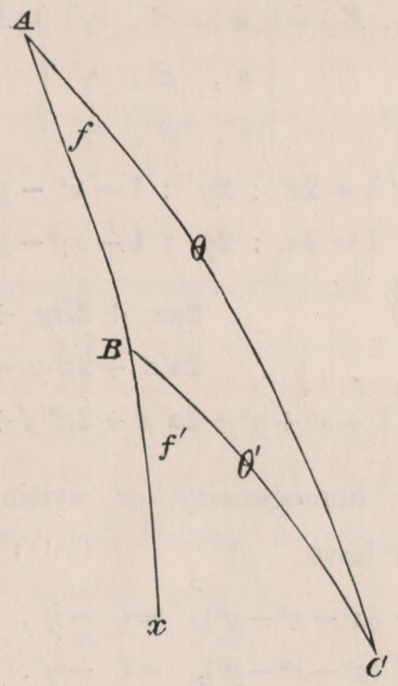

next in regard to $B$ by its distance $\theta^{\prime}$ and azimuth $f^{\prime}$, the azimuths from the great circle $A B x$ which joins the two points $A$ and $B$, then we have

$$
\tan \frac{1}{2} \theta(\cos f+i \sin f) \text {, and } \tan \frac{1}{2} \theta^{\prime}\left(\cos f^{\prime}+i \sin f^{\prime}\right),
$$

homographic functions one of the other: calling them $s, s^{\prime}$, and putting the distance $A B=c$, the relation between them in fact is

or, what is the same thing,

$$
s^{\prime}=\frac{s-\tan \frac{1}{2} c}{1+s \tan \frac{1}{2} c},
$$

or, observing that

$$
\tan \frac{1}{2} c\left(1+s s^{\prime}\right)=s-s^{\prime} ;
$$

$$
s s^{\prime}=\tan \frac{1}{2} \theta \tan \frac{1}{2} \theta^{\prime}\left\{\cos \left(f+f^{\prime}\right)+i \sin \left(f+f^{\prime}\right)\right\},
$$

we have the two equations

$$
\begin{aligned}
& \tan \frac{1}{2} c\left\{1+\tan \frac{1}{2} \theta \tan \frac{1}{2} \theta^{\prime} \cos \left(f+f^{\prime}\right)\right\}=\tan \frac{1}{2} \theta \cos f-\tan \frac{1}{2} \theta^{\prime} \cos f^{\prime}, \\
& \tan \frac{1}{2} c\left\{\quad \tan \frac{1}{2} \theta \tan \frac{1}{2} \theta^{\prime} \sin \left(f+f^{\prime}\right)\right\}=\tan \frac{1}{2} \theta \sin f-\tan \frac{1}{2} \theta^{\prime} \sin f^{\prime} .
\end{aligned}
$$

88. If we denote the angles of the spherical triangle by $C, A, B$, and the opposite sides by $c$ (as before), $a, b$, then $\theta, \theta^{\prime}=b, a ; f, f^{\prime}=A, \pi-B$, whence

$$
s, s^{\prime}=\tan \frac{1}{2} b(\cos A+i \sin A), \quad-\tan \frac{1}{2} a(\cos B-i \sin B):
$$

or we have between the sides $a, b, c$ and angles $A, B$ of a spherical triangle the relations

$\tan \frac{1}{2} c\left\{1-\tan \frac{1}{2} a \tan \frac{1}{2} b \cos (A-B)\right\}=\tan \frac{1}{2} b \cos A+\tan \frac{1}{2} a \cos B$, $\tan \frac{1}{2} c\left\{-\tan \frac{1}{2} a \tan \frac{1}{2} b \sin (A-B)\right\}=\tan \frac{1}{2} b \sin A-\tan \frac{1}{2} a \sin B$; 
equations which may be verified by means of the ordinary formulæ of Spherical Trigonometry.

89. But it is interesting to give the proof with rectangular coordinates.

Taking $(X, Y, Z),\left(X_{1}, Y_{1}, Z_{1}\right)$ for the coordinates, referred to two different sets of axes, of a point on the spherical surface: also $x, y, x_{1}, y_{1}$ for the coordinates of the corresponding stereographic projections, we have

and thence

$$
\begin{aligned}
& \left(X_{1}, Y_{1}, Z_{1}\right)=\left(\begin{array}{ccc}
\alpha, & \beta, & \gamma \\
\alpha^{\prime}, & \beta^{\prime}, & \gamma^{\prime} \\
\alpha^{\prime \prime}, & \beta^{\prime \prime}, & \gamma^{\prime \prime}
\end{array} \mid\right. \\
& X: Y: Z: 1=2 x: 2 y: 1-x^{2}-y^{2}: 1+x^{2}+y^{2}, \\
& X_{1}: Y_{1}: Z_{1}: 1=2 x_{1}: 2 y_{1}: 1-x_{1}{ }^{2}-y_{1}{ }^{2}: 1+x_{1}{ }^{2}+y_{1}{ }^{2},
\end{aligned}
$$

$$
\begin{aligned}
& x_{1}: y_{1}: 1= \\
& 2 \alpha x+2 \beta y+\gamma\left(1-x^{2}-y^{2}\right) \\
& 2 \alpha^{\prime} x+2 \beta^{\prime} y+\gamma^{\prime}\left(1-x^{2}-y^{2}\right) \\
& \text { : } 1+x^{2}+y^{2}+2 \alpha^{\prime \prime} x+2 \beta^{\prime \prime} y+\gamma^{\prime \prime}\left(1-x^{2}-y^{2}\right) \text {. }
\end{aligned}
$$

90. Introducing $z, z_{1}$ for homogeneity, or writing $\frac{x}{z}, \frac{y}{z}$ and $\frac{x_{1}}{z_{1}}, \frac{y_{1}}{z_{1}}$ in place of $x, y$ and $x_{1}, y_{1}$, respectively, we have

$x_{1}=\quad 2 \alpha x+2 \beta y+\gamma\left(z^{2}-x^{2}-y^{2}\right),=\left(-\gamma,-\gamma, \quad \gamma, \beta, \alpha, 0 \gamma(x, y, z)^{2}\right.$, $y_{1}=\quad 2 \alpha^{\prime} x+2 \beta^{\prime} y+\gamma^{\prime}\left(z^{2}-x^{2}-y^{2}\right),=\left(-\gamma^{\prime},-\gamma^{\prime}, \quad \gamma^{\prime}, \beta^{\prime}, \alpha^{\prime}, 0 \gamma \quad\right)^{2}$, $z_{1}=z^{2}+x^{2}+y^{2}+2 \alpha^{\prime \prime} x+2 \beta^{\prime \prime} y+\gamma^{\prime \prime}\left(z^{2}-x^{2}-y^{2}\right), \quad=\left(1-r^{\prime \prime}, 1-\gamma^{\prime \prime}, 1+\gamma^{\prime \prime}, \beta^{\prime \prime}, \alpha^{\prime \prime}, 0 \gamma \quad \text { ” }\right)^{2}$, and thence without difficulty

$$
\begin{aligned}
z_{1} & =\frac{1}{1+\gamma^{\prime \prime}}\left\{\left(1+\gamma^{\prime \prime}\right) z+\left(\alpha^{\prime \prime}+i \beta^{\prime \prime}\right)(x-i y)\right\}\left\{\left(1+\gamma^{\prime \prime}\right) z+\left(\alpha^{\prime \prime}-i \beta^{\prime \prime}\right)(x+i y)\right\}, \\
x_{1}+i y_{1} & =\frac{1}{\gamma+\gamma^{\prime 2}}\left\{\left(1+\gamma^{\prime \prime}\right) z+\left(\alpha^{\prime \prime}+i \beta^{\prime \prime}\right)(x-i y)\right\}\left\{\left(1-\gamma^{\prime \prime}\right) z+\left(-\alpha^{\prime \prime}+i \beta^{\prime \prime}\right)(x+i y)\right\}, \\
x_{1}-i y_{1} & =\frac{1}{\gamma-\gamma^{\prime 2}}\left\{\left(1-\gamma^{\prime \prime}\right) z-\left(\alpha^{\prime \prime}+i \beta^{\prime \prime}\right)(x-i y)\right\}\left\{\left(1+\gamma^{\prime \prime}\right) z+\left(\alpha^{\prime \prime}-i \beta^{\prime \prime}\right)(x+i y)\right\},
\end{aligned}
$$

viz. the form is $z_{1}: x_{1}+i y_{1}: x_{1}-i y_{1}=M N: N L: L M(L, M, N$ linear functions of $z, x+i y, x-i y)$ : showing that the relation between two stereographic projections of the same spherical figure is in fact that of a quadric transformation, the fundamental points in each figure being an arbitrary point and the two circular points at infinity: or, what is the same thing, to any line in the one figure there corresponds a circle in the other figure, which is the "circular relation" of Möbius.

91. The actual values are

$$
\begin{aligned}
& \frac{x_{1}+i y_{1}}{z_{1}}=\frac{1+\gamma^{\prime \prime}}{\gamma+\gamma^{\prime} i} \cdot \frac{\left(1-\gamma^{\prime \prime}\right) z-\left(\alpha^{\prime \prime}-i \beta^{\prime \prime}\right)(x+i y)}{\left(1+\gamma^{\prime \prime}\right) z+\left(\alpha^{\prime \prime}-i \beta^{\prime \prime}\right)(x+i y)}, \\
& \frac{x_{1}-i y_{1}}{z_{1}}=\frac{1+\gamma^{\prime \prime}}{\gamma-\gamma^{\prime} i} \cdot \frac{\left(1-\gamma^{\prime \prime}\right) z-\left(\alpha^{\prime \prime}+i \beta^{\prime \prime}\right)(x-i y)}{\left(1+\gamma^{\prime \prime}\right) z+\left(\alpha^{\prime \prime}+i \beta^{\prime \prime}\right)(x-i y)},
\end{aligned}
$$


viz. attending only to the former of these, we have $\frac{x_{1}+i y_{1}}{z_{1}}$ a homographic function of $\frac{x+i y}{z}$, which is the before-mentioned theorem.

92. Supposing that the transformation from $(X, Y, Z)$ to $\left(X_{1}, Y_{1}, Z_{1}\right)$ is made by a rotation, the coordinates of which are $\lambda, \mu, \nu$ : that is, if $f, g, h$ are the inclinations of the resultant axis to the axes of $x, y, z$ respectively, and $\theta$ the angle of rotation, putting $\lambda, \mu, \nu=\tan \frac{1}{2} \theta \cos f, \tan \frac{1}{2} \theta \cos g, \tan \frac{1}{2} \theta \cos h$ : then the coefficients of transformation are

$$
\left(\begin{array}{ccc}
\alpha, & \beta, & \gamma \\
\alpha^{\prime}, & \beta^{\prime}, & \gamma^{\prime} \\
\alpha^{\prime \prime}, & \beta^{\prime \prime}, & \gamma^{\prime \prime}
\end{array}\left|\begin{array}{ccc}
1+\lambda^{2}-\mu^{2}-\nu^{2}, & 2(\lambda \mu+\nu), & 2(\lambda \nu-\mu) \\
2(\mu \lambda-\nu), & 1-\lambda^{2}+\mu^{2}-\nu^{2}, & 2(\mu \nu+\lambda) \\
2(\nu \lambda+\mu), & 2(\mu \nu-\lambda) \quad, & 1-\lambda^{2}-\mu^{2}+\nu^{2}
\end{array}\right| \div\left(1+\lambda^{2}+\mu^{2}+\nu^{2}\right)\right.
$$

Substituting these values, the formulæ become, after an easy reduction,

$$
\begin{aligned}
& \frac{x_{1}+i y_{1}}{z_{1}}=\frac{-(\nu+i)(x+i y)+(\lambda+i \mu) z}{(\lambda-i \mu)(x+i y)+(\nu-i) z}, \\
& \frac{x_{1}-i y_{1}}{z_{1}}=\frac{-(\nu-i)(x-i y)+(\lambda-i \mu) z}{(\lambda+i \mu)(x-i y)+(\nu+i) z} ;
\end{aligned}
$$

attending to the former of these, and writing for greater simplicity

respectively, we have

$$
\frac{x_{1}+i y_{1}}{z_{1}}, \frac{x+i y}{z}=s_{1}, s
$$

or writing this

$$
s_{1}=\frac{-(\nu+i) s+(\lambda+i \mu)}{(\lambda-i \mu) s+(\nu-i)},
$$

$$
s_{1}=\frac{A s+B}{C s+D},
$$

then

$$
A: B: C: D=-\nu-i: \lambda+i \mu: \lambda-i \mu: \nu-i \text {. }
$$

93. I call to mind that the condition, in order that the homographic transformation $s_{1}=(A s+B) \div(C s+D)$ may be periodic of the order $n$, is

$$
(A+D)^{2}-4(A D-B C) \cos ^{2} \frac{m \pi}{n}=0,
$$

$m$ being an integer different from zero and prime to $n$. In particular, when $n=2$, it is $A+D=0: n=3$, it is $A^{2}+A D+D^{2}+B C=0: n=4$, it is $A^{2}+D^{2}+2 B C=0:$ and $n=5$, it is $(A+D)^{2}-\frac{1}{2}(3 \pm \sqrt{ } 5)(A D-B C)=0$.

Groups of homographic transformations. Art. Nos. 94 and 95.

94. The formulæ just obtained serve to connect the theory of the rotations of a polyhedron with that of the homographic transformations $s$ into $(A s+B) \div(C s+D)$ : and, corresponding to the rotations which leave the polyhedron unaltered, we have groups of homographic transformations. We have thus, corresponding to the cases of the tetrahedron, the cube and the octahedron, and the dodecahedron and icosahedron respectively, groups of 12 , of 24 , and of 60 homographic transformations $s$ into 
$(A s+B) \div(C s+D)$. The group of 60 and the group of 24 include each of them as part of itself the group of 12: it is further to be remarked that the group of 12 may be regarded as that of the positive substitutions upon four letters abcd, the group of 24 as that of all the substitutions upon the four letters, and the group of 60 as that of the positive substitutions upon five letters abcde.

95. I call to mind that a group of functional symbols $1, \alpha, \beta, \ldots$ can always be expressed in the equivalent form $1,2 a 9^{-1}, 9 \beta 9^{-1}, \ldots$ where $\vartheta$ is any functional symbol whatever: clearly, $\alpha, \beta, \ldots$ being homographic transformations, then, 9 being any homographic transformation whatever, the new symbols $9 \alpha 9^{-1}, 9 \beta 9^{-1}, \ldots$ will also be homographic transformations; and thus the group of homographic transformations can be expressed in various equivalent forms: these correspond to the different positions of the polyhedron in regard to the axes of coordinates: and there are in fact three cases which it is proper to consider, viz. attending for the moment to the dodecahedron, we may have the axis of $z$ passing through the midpoint of a side, through the centre of a face, or through a summit; that is, in the language presently explained, the cases are $1^{\circ}$, Pole at a point $\Theta ; 2^{\circ}$, Pole at a point $A$; $3^{\circ}$, Pole at a point $B$.

The regular Polyhedra. Art. Nos. 96 to 103.

96. We require a theory of the regular Polyhedra considered as systems of points on a sphere. I refer to my two papers [375] and [679]. In the latter paper, I remark that, considering the five regular figures drawn in proper relation to each other on the same spherical surface, the only points which have to be considered are 12 points $A, 20$ points $B, 30$ points $\Theta$, and $6^{n}$ points $\Phi$. Describing these by reference to the dodecahedron, the points $A$ are the centres of the faces, the points $B$ are the summits, the points $\Theta$ are the midpoints of the sides, and the points $\Phi$ are the midpoints of the diagonals of the faces. Or describing them by reference to the icosahedron, the points $A$ are the summits, the points $B$ are the centres of the faces, the points $\Theta$ are the midpoints of the sides: viz. each point $\Theta$ is the common midpoint of a side of the dodecahedron and a side of the icosahedron, which there intersect at right angles: and the points $\Phi$ are points lying by threes on the faces of the icosahedron, each point $\Phi$ of the face being given as the intersection of a perpendicular $A \Theta$ of the face by a line $B B$ joining the centres of two adjacent faces and which intersects $A \Theta$ at right angles.

97. The points $\Phi$ are comparatively unimportant, and it is proper in the first instance to attend only to the 12 points $A$, the 20 points $B$, and the 30 points $\Theta$ : these form 6 pairs of opposite points $A, 10$ pairs of opposite points $B$, and 15 pairs of opposite points $\Theta$. Considering the diameters through each pair of opposite points $\Theta$, we have thus a system of 15 axes, which in fact form 5 sets each of 3 rectangular axes: attending to any one of such sets, the diametral plane at right angles to one of the three axes contains of course the other two axes: it contains also two axes each through a pair of opposite points $A$, and two axes each through a pair of opposite points $B$. If instead of the plane we consider its intersection with the sphere, we have thus on the sphere 15 circles each containing 4 points $\Theta$, 
4 points $A$ and 4 points $B$. The fifteen circles intersect by fives in the pairs of opposite points $A$, by threes in the pairs of opposite points $B$, and by twos in the pairs of opposite points $\Theta$; the mutual inclinations of successive circles at the points $A, B, \Theta$ being $=36^{\circ}, 60^{\circ}$ and $90^{\circ}$ respectively. The whole number $15.14,=210$, of the intersections of the circles two and two together is thus made up of the 12 points $A$ each counting 10 times, the 20 points $B$ each counting 3 times, and the 30 points $\Theta$ each counting once; $210=120+60+30$.

98. The angular magnitudes which present themselves are all obtained from the dodecahedral pentagon, as shown in the annexed figure, in which the angle subtended by a side at the centre is $=72^{\circ}$, and the angle between two adjacent sides is $=120^{\circ}$.

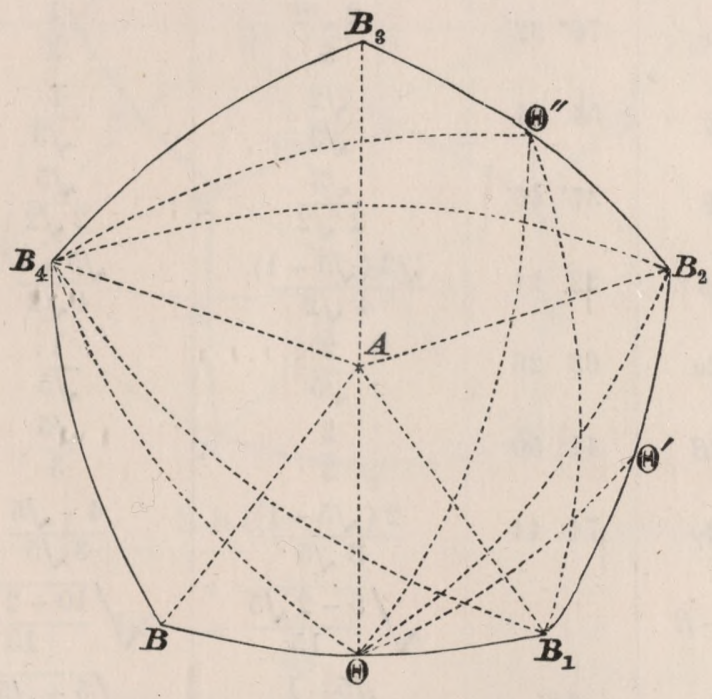

We write $A \Theta=\alpha, B \Theta=\beta, A B=\gamma, B_{1} B_{4}=x, \angle B_{1} B_{4} B=\theta, \Theta B_{4}=g, \angle \Theta B_{4} B=\phi$.

From the triangle $A \Theta B$, the angles of which are $36^{\circ}, 90^{\circ}, 60^{\circ}$ and the opposite sides $\beta, \gamma, \alpha$, we find the values of $\alpha, \beta, \gamma$, and these are such that $\alpha+\beta+\gamma=\frac{1}{2} \pi$.

From the triangle $B_{4} B B_{1}$, where the sides $B_{4} B, B B_{1}$, and the included angle are $2 \beta, 2 \beta, 120^{\circ}$, we have the opposite side $x$, and the other two angles each $=\theta$.

From the triangle $B_{4} B \Theta$, where the sides $B_{4} B, B \Theta$, and the included angle are $2 \beta, \beta, 120^{\circ}$, we find the opposite side $g$, the angle $B B_{4} \Theta,=\phi$, and the angle $B_{4} \Theta B,=45^{\circ}$.

Hence each of the angles $B_{4} \Theta B, B_{2} \Theta B_{1}$, being $=45^{\circ}$, the angle $B_{4} \Theta B_{2}$ is $=90^{\circ}$ : in this triangle the hypothenuse $B_{2} B_{4}$ is $=x$, and each of the other two sides is $=g$ : whence we have $\cos x=\cos ^{2} g$, as is in fact the case, and moreover the values give $x+2 g=180^{\circ}$. Also each of the other angles is found to be $=60^{\circ}$; that is, we have $\angle B_{2} B_{4} \Theta=60^{\circ}$, or the whole angle at $B_{4}$ being $=120^{\circ}$, the sum of the remaining angles $B_{3} B_{4} B_{2}$ and $B B_{4} \Theta$ is $=60^{\circ}$ : that is, $\theta+\phi=60^{\circ}$.

From the triangle $\Theta B_{1} \Theta^{\prime}$ where the two sides and the included angle are $\beta, \beta, 120^{\circ}$, we find $\Theta \Theta^{\prime}=36^{\circ}$. 
And from the triangle $\Theta B_{4} \Theta^{\prime \prime}$, where the two sides and the included angle are $g, g$ and $\left(120^{\circ}-2 \phi=\right) 2 \theta$, we find $\Theta \Theta^{\prime \prime}=60^{\circ}$.

99. We thus arrive at the following Table:

\begin{tabular}{|c|c|c|c|c|}
\hline & & & $\sin$ & $\cos$ \\
\hline$A \Theta$ & $\alpha$ & $31^{\circ} 43^{\prime}$ & $\sqrt{\frac{5-\sqrt{ } 5}{10}}$ & $\frac{5+\sqrt{ } 5}{10}$ \\
\hline$B \Theta$ & $\beta$ & $20^{\circ} 55^{\prime}$ & $\begin{array}{c}\sqrt{ } 5-1 \\
2 \sqrt{ } 3\end{array}$ & $\frac{\sqrt{ } 5+1}{2 \sqrt{ } 3}$ \\
\hline$A B$ & $\gamma$ & $37^{\circ} 22^{\prime}$ & $\sqrt{\frac{10-2 \sqrt{ } 5}{15}}$ & $\frac{5+2 \sqrt{ } 5}{15}$ \\
\hline$(B B)$ & $x$ & $70^{\circ} 32^{\prime}$ & $\frac{2 \sqrt{ } 2}{3}$ & $\frac{1}{3}$ \\
\hline$(B \Theta)$ & $g$ & $54 \quad 44$ & $\frac{\sqrt{ } 2}{\sqrt{ } 3}$ & $\frac{1}{\sqrt{ } 3}$ \\
\hline$B B B$ & $\theta$ & $37^{\circ} 46^{\prime}$ & $\frac{\sqrt{ } 3}{2 \sqrt{ } 2}$ & $\frac{\sqrt{ } 5}{2 \sqrt{ } 2}$ \\
\hline$B \Theta B$ & $\phi$ & $22 \quad 14$ & $\frac{\sqrt{ } 3(\sqrt{ } 5-1)}{4 \sqrt{ } 2}$ & $\frac{\sqrt{5}+3}{4 \sqrt{2}}$ \\
\hline & $2 \alpha$ & $\begin{array}{ll}63 & 26\end{array}$ & $\frac{2}{\sqrt{ } 5}$ & $\frac{1}{\sqrt{ } 5}$ \\
\hline & $2 \beta$ & 4150 & $\frac{2}{3}$ & $\frac{\sqrt{ } 5}{3}$ \\
\hline & $2 \gamma$ & $74 \quad 44$ & $\frac{2(\sqrt{ } 5+1)}{3 \sqrt{ } 5}$ & $\frac{4-\sqrt{ } 5}{3 \sqrt{ } 5}$ \\
\hline & $\alpha-\beta$ & & $\sqrt{\frac{5-2 \sqrt{5}}{15}}$ & $\frac{10+2 \sqrt{0}}{15}$ \\
\hline & & $18^{\circ}$ & $\frac{\sqrt{5}-1}{4}$ & $\frac{5+\sqrt{ } 5}{8}$ \\
\hline$\Theta \Theta$ & & $36^{\circ}$ & $\sqrt{\frac{5-\sqrt{ } 5}{8}}$ & $\frac{\sqrt{5}+1}{4}$ \\
\hline
\end{tabular}

where as above

$$
\begin{aligned}
& \alpha+\beta+\gamma=90^{\circ}, \\
& x+2 g=180^{\circ}, \\
& \theta+\phi \quad=60^{\circ} .
\end{aligned}
$$

100. We now construct three figures of the points $A, B, \Theta$; viz. these are stereographic projections, each showing the Northern hemisphere projected on the plane of the equator by lines drawn to the South Pole: hence, for any pair of opposite points not on the equator, only the point in the Northern hemisphere is shown: but for a pair of opposite points on the equator the two points are each of them shown. In fig. 1 the North Pole is taken to be a point $\Theta$; in fig. 2 it is a point $A$; and in fig. 3 it is a point $B$. The position of any point on the sphere is determined by its N.P.D. and its longitude, measured from an arbitrary origin, say from the point $E$ of the centre left-handedly: then, in the three figures, the positions are as follows. 
101. Fig. 1. Pole at $\Theta$.

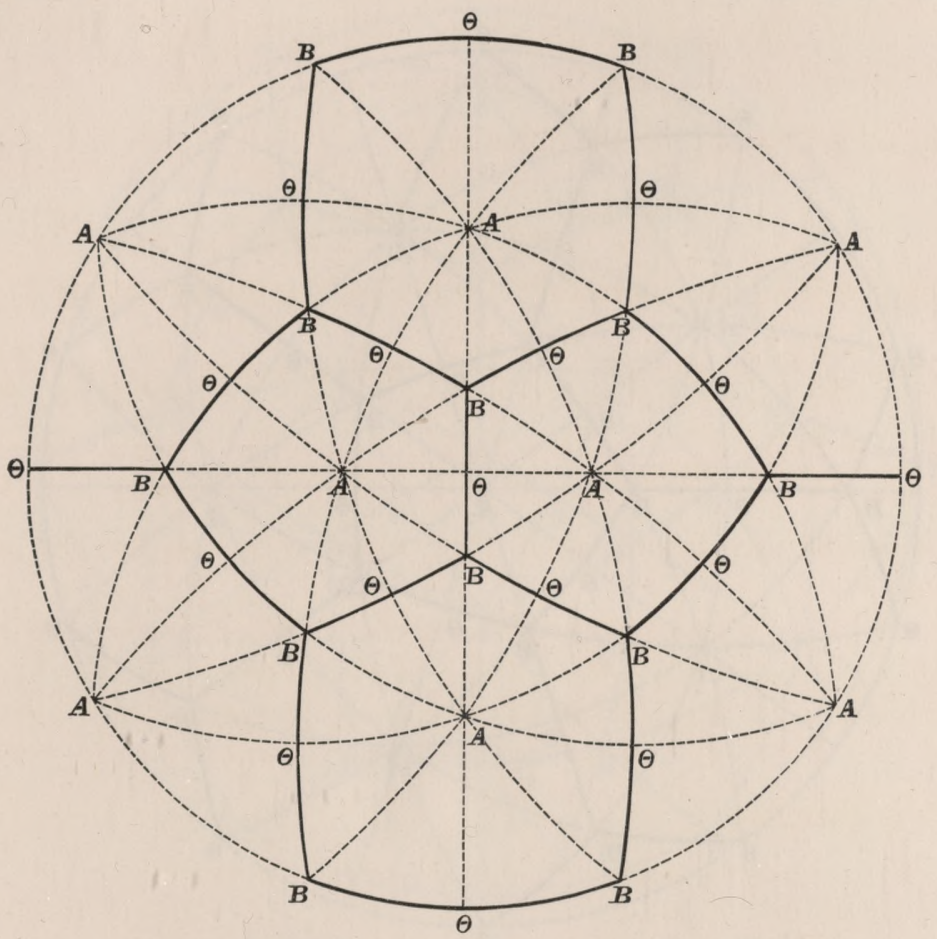

N.P.D.'s

Longitudes.

\begin{tabular}{|r|r|c|}
\hline $2 A$ & $a=31^{\circ} 43^{\prime}$ & $0^{\circ}, 180^{\circ}$ \\
$2 A$ & $90^{\circ}-\alpha=5817$ & 90,270 \\
$4 A$ & 90 & $(0,180) \pm \alpha=31^{\circ} 43^{\prime}$ \\
$2 A$ & $90^{\circ}+\alpha=12143$ & 90,270 \\
$2 A$ & $180^{\circ}-\alpha=14817$ & 0,180 \\
\hline $2 B$ & $\beta=20^{\circ} 55^{\prime}$ & $90^{\circ}, 270^{\circ}$ \\
$4 B$ & $g=5444$ & $45,135,225,315$ \\
$2 B$ & $90^{\circ}-\beta=695$ & 0,180 \\
$4 B$ & 90 & $(90,270) \pm \beta=20^{\circ} 55^{\prime}$ \\
$2 B$ & $90^{\circ}+\beta=11055$ & 0,180 \\
$4 B$ & $180^{\circ}-g=12516$ & $45,135,225,315$ \\
$2 B$ & $180^{\circ}-\beta=1595$ & 90,270 \\
\hline $1 \Theta$ & 0 & - \\
$4 \Theta$ & $0^{\circ}$ & $\left(90^{\circ}, 270^{\circ}\right) \pm \alpha=31^{\circ} 43^{\prime}$ \\
$4 \Theta$ & 60 & $(0,180) \pm \beta=2055$ \\
$4 \Theta$ & 72 & $(90,270) \pm \alpha=3143$ \\
$4 \Theta$ & 90 & $0,90,180,270$ \\
$4 \Theta$ & 108 & $(90,270) \pm \alpha=3143$ \\
$4 \Theta$ & 120 & $(0,180) \pm \beta=2055$ \\
$4 \Theta$ & 144 & $(90,270) \pm \alpha=3143$ \\
$1 \Theta$ & 180 & - \\
\hline
\end{tabular}

C. XI. 
102. Fig. 2. Pole at $A$.

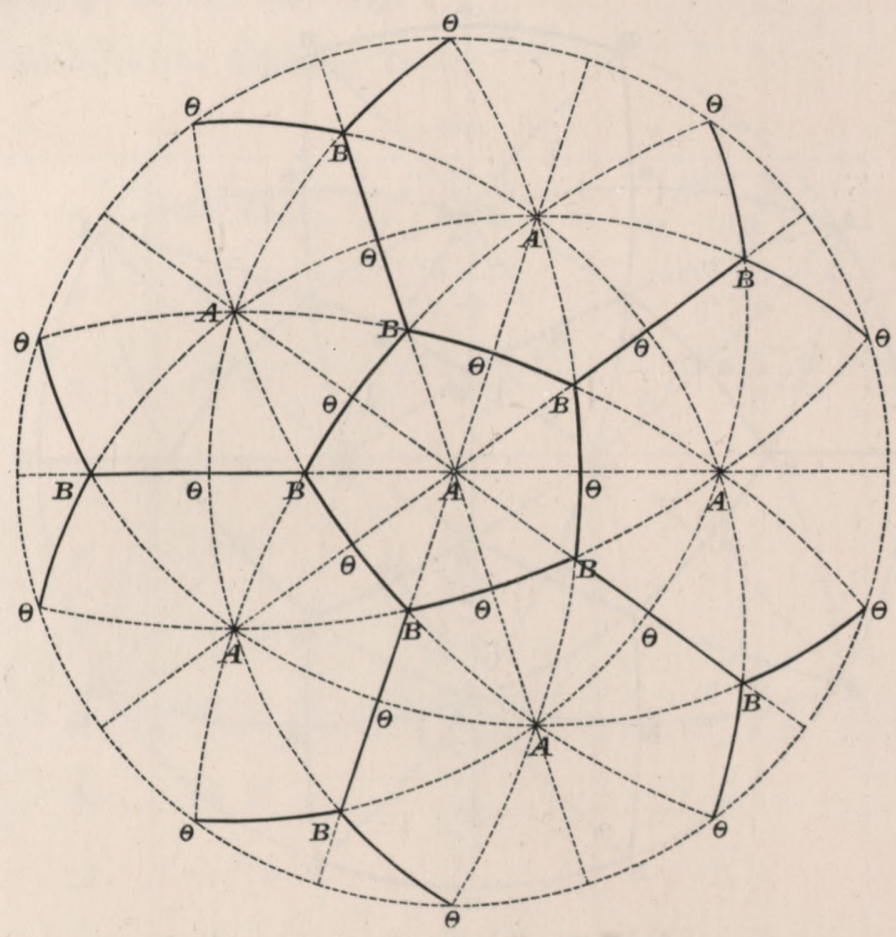

N.P.D.'s

Longitudes.

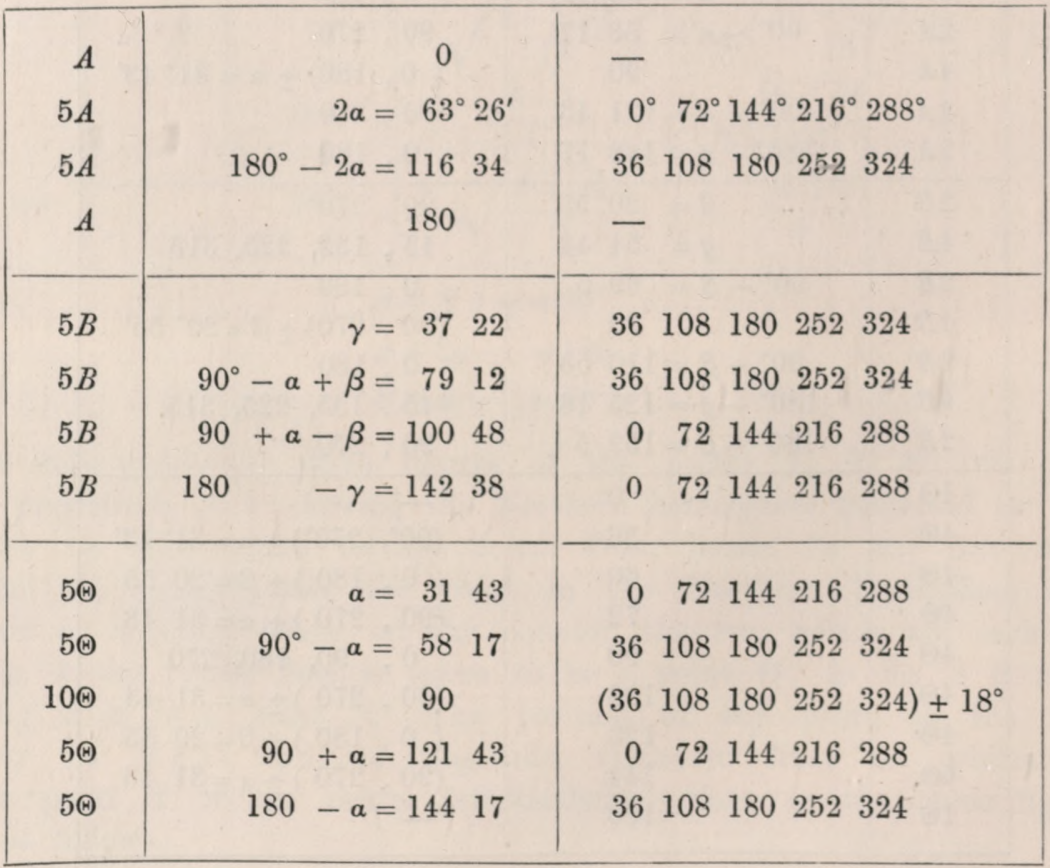


103. Fig. 3. Pole at $B$.

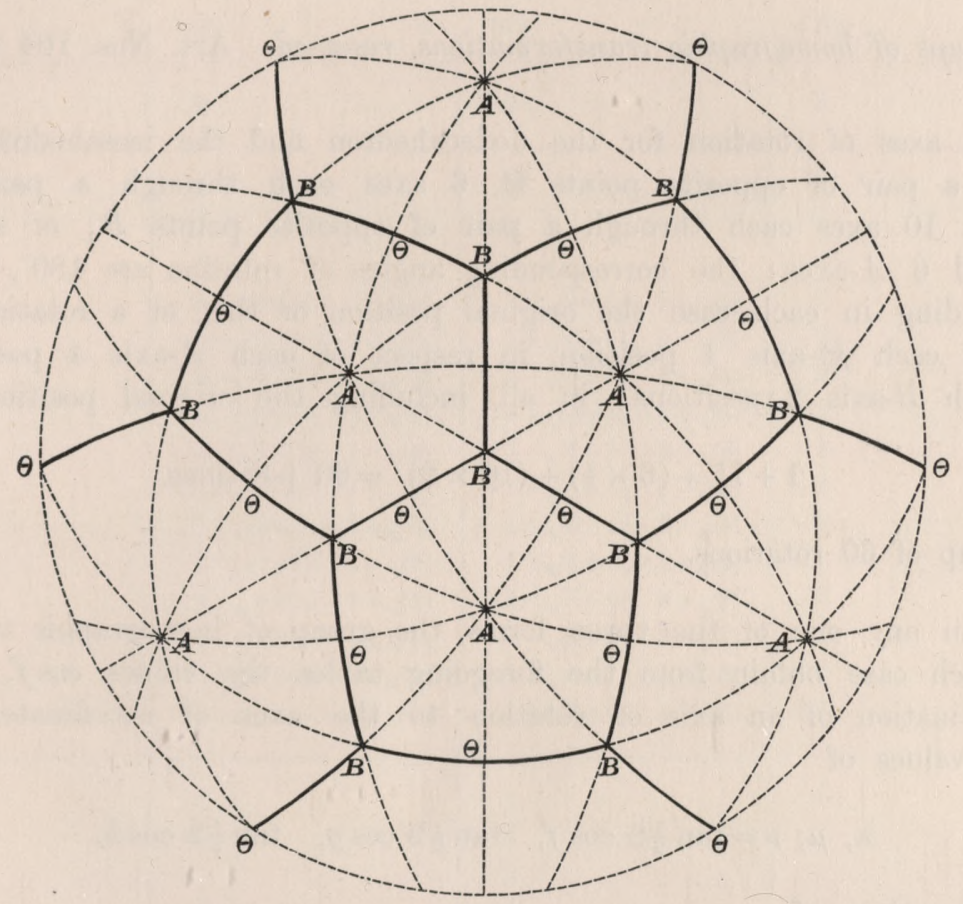

N.P.D.'s

Longitudes.

\begin{tabular}{|c|c|c|}
\hline $\begin{array}{l}3 A \\
3 A \\
3 A \\
3 A\end{array}$ & $\begin{aligned} \gamma & =37^{\circ} 22^{\prime} \\
90^{\circ}-\alpha+\beta & =7912 \\
90+\alpha-\beta & =10048 \\
180-\gamma & =14238\end{aligned}$ & $\begin{array}{lll}30^{\circ} & 150^{\circ} & 270^{\circ} \\
90 & 210 & 330 \\
30 & 150 & 270 \\
90 & 210 & 330\end{array}$ \\
\hline $\begin{array}{r}B \\
3 B \\
6 B \\
6 B \\
3 B \\
B\end{array}$ & 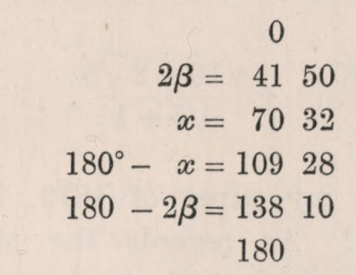 & 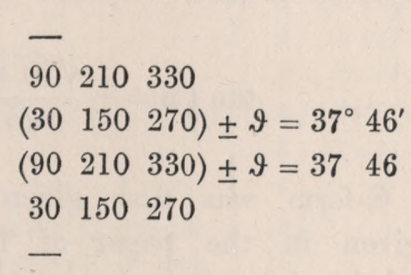 \\
\hline $\begin{array}{l}3 \Theta \\
6 \Theta \\
3 \Theta \\
6 \Theta \\
3 \Theta \\
6 \Theta \\
3 \Theta\end{array}$ & 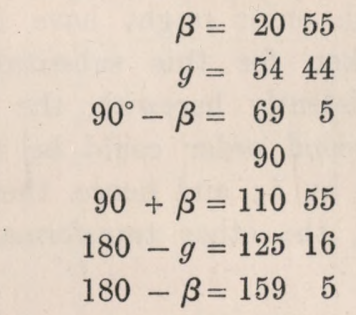 & 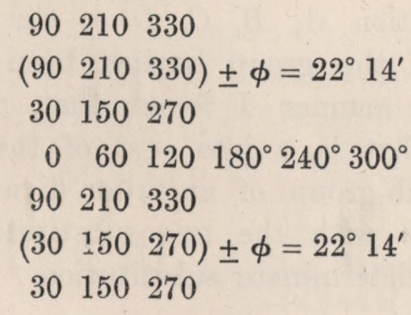 \\
\hline
\end{tabular}


The groups of homographic transformations, resumed. Art. Nos. 104 to 117.

104. The axes of rotation for the dodecahedron and the icosahedron are 15 axes each through a pair of opposite points $\Theta, 6$ axes each through a pair of opposite points $A$, and 10 axes each through a pair of opposite points $B$; or say $15 \Theta$-axes, $10 B$-axes and $6 A$-axes: the corresponding angles of rotation are $180^{\circ}, 72^{\circ}$ and $120^{\circ}$; so that (excluding in each case the original position or that of a rotation 0 ) we have in respect of each $\Theta$-axis 1 position, in respect of each $A$-axis 4 positions, and in respect of each $B$-axis 2 positions; in all, including the original position,

$$
1+15+(6 \times 4)+(10 \times 2),=60 \text { positions, }
$$

that is, a group of 60 rotations.

To find, in any one of the three forms, the group of homographic transformations, we can in each case obtain from the foregoing tables the values $\cos f, \cos g, \cos h$ of the cosine-inclination of an axis of rotation to the axes of coordinates, and thence calculate the values of

$$
\lambda, \mu, \nu=\tan \frac{1}{2} 9 \cos f, \quad \tan \frac{1}{2} 9 \cos g, \quad \tan \frac{1}{2} 9 \cos h,
$$

and thence the values of

$$
A, B, C, D=-\nu-i, \quad \lambda+i \mu, \quad \lambda-i \mu, \quad \nu-i
$$

viz. in the case of a $\Theta$-axis, 9 is $=180^{\circ}$, (so that here $\tan \frac{1}{2} 9=\infty$, or the values of $A, B, C, D$ are $=-\nu, \lambda+i \mu, \lambda-i \mu, \nu$, that is, $-\cos h, \cos f+i \cos g, \cos f-i \cos g, \cos h)$; in the case of a $B$-axis, the values are $9=120^{\circ}, 240^{\circ}$, and therefore $\tan \frac{1}{2} 9= \pm \sqrt{ } 3$; and in the case of an $A$-axis, they are $9=72^{\circ}, 144^{\circ}, 216^{\circ}, 288^{\circ}$, and therefore

$$
\tan \frac{1}{2} 9= \pm \frac{\sqrt{10+2 \sqrt{ } 5}}{\sqrt{ } 5-1}, \pm \frac{\sqrt{10-2 \sqrt{ } 5}}{\sqrt{ } 5+1}
$$

105. The $\Theta$-form was first given in my paper of 1879 , but in obtaining it I used results given in the paper of 1877. As regards the identification with the substitution-symbols, since there is nothing to distinguish inter se the letters $a, b, c, d, e$, any transformation $A, B, C, D$ of the fifth order might have been taken for abcde, but No. 37 of the group having been taken for this substitution abcde, I do not recall in what manner $\mathrm{I}$ found that, consistently herewith, the transformation No. 2 $(-1,0,0,1$, that is, $s$ into $-s)$ of the second order could be taken for ab.cd. But there is no sub-group of an order divisible by 5 ; and hence, these two transformations being identified with the two substitutions, the other transformations correspond each of them to a determinate substitution. 
106. Homographic Transformations. The group of 60 . Pole at $\Theta$.

$\left(\begin{array}{llll}(A x & +B) & \div(C x & +D\end{array}\right)$

\begin{tabular}{|c|c|c|c|c|c|}
\hline 1 & 1 & 0 & , & 1 & 1 \\
\hline 2 & -1 & 0 & 0 & 1 & $a b \cdot c d$ \\
\hline 3 & 0 & 1 & 1 & 0 & $a c \cdot b d$ \\
\hline 4 & 0 & 1 & -1 & 0 & $a d . b c$ \\
\hline 5 & 2 & $-3+\sqrt{ } 5+i(1-\sqrt{ } 5)$ & $-3+\sqrt{ } 5+i(-1+\sqrt{ } 5)$ & -2 & $b c . d e$ \\
\hline 6 & 2 & $-3+\sqrt{ } 5+i(-1+\sqrt{ } 5)$ & $-3+\sqrt{ } 5+i(1-\sqrt{ } 5)$ & -2 & $a e \cdot b c$ \\
\hline 7 & 2 & $3-\sqrt{ } 5+i(-1+\sqrt{ } 5)$ & $3-\sqrt{ } 5+i(1-\sqrt{ } 5)$ & -2 & ad.ce \\
\hline 8 & 2 & $3-\sqrt{ } 5+i(1-\sqrt{ } 5)$ & $3-\sqrt{ } 5+i(-1+\sqrt{ } 5)$ & -2 & $a d . b e$ \\
\hline 9 & 2 & $-1-\sqrt{ } 5+i(1-\sqrt{ } 5)$ & $-1-\sqrt{ } 5+i(-1+\sqrt{ } 5)$ & -2 & $a e . c d$ \\
\hline 10 & 2 & $-1-\sqrt{ } 5+i(-1+\sqrt{ } 5)$ & $-1-\sqrt{ } 5+i(1-\sqrt{ } 5)$ & -2 & $a b \cdot d e$ \\
\hline 11 & 2 & $1+\sqrt{ } 5+i(-1+\sqrt{ } 5)$ & $1+\sqrt{ } 5+i(1-\sqrt{ } 5)$ & -2 & $b e . c d$ \\
\hline 12 & 2 & $1+\sqrt{ } 5+i(1-\sqrt{ } 5)$ & $1+\sqrt{ } 5+i(-1+\sqrt{ } 5)$ & -2 & $a b \cdot c e$ \\
\hline 13 & 2 & $-1-\sqrt{ } 5+i(-3-\sqrt{ } 5)$ & $-1-\sqrt{ } 5+i(3+\sqrt{ } 5)$ & -2 & $a c . b e$ \\
\hline 14 & 2 & $-1-\sqrt{ } 5+i(3+\sqrt{ } 5)$ & $-1-\sqrt{ } 5+i(-3-\sqrt{ } 5)$ & -2 & $b d . c e$ \\
\hline 15 & 2 & $1+\sqrt{ } 5+i(3+\sqrt{ } 5)$ & $1+\sqrt{ } 5+i(-3-\sqrt{ } 5)$ & -2 & $a e \cdot b d$ \\
\hline 16 & 2 & $1+\sqrt{ } 5+i(-3-\sqrt{ } 5)$ & $1+\sqrt{ } 5+i(3+\sqrt{ } 5)$ & -2 & ac.de \\
\hline 17 & $-i$ & $i$ & 1 & 1 & $a b c$ \\
\hline 18 & -1 & $i$ & 1 & $i$ & $a c b$ \\
\hline 19 & 1 & $-i$ & 1 & $i$ & $a d c$ \\
\hline 20 & $-i$ & $-i$ & 1 & -1 & acd \\
\hline 21 & $i$ & $i$ & 1 & -1 & $a d b$ \\
\hline 22 & 1 & $i$ & 1 & $-i$ & $a b d$ \\
\hline 23 & -1 & $-i$ & 1 & $-i$ & $b c d$ \\
\hline 24 & $i$ & $-i$ & 1 & 1 & $b d c$ \\
\hline 25 & $-1-\sqrt{ } 5+i(3+\sqrt{ } 5)$ & 2 & -2 & $-1-\sqrt{ } 5+i(-3-\sqrt{ } 5)$ & aec \\
\hline 26 & $1+\sqrt{ } 5+i(3+\sqrt{ } 5)$ & 2 & -2 & $1+\sqrt{ } 5+i(-3-\sqrt{ } 5)$ & ace \\
\hline 27 & $1+\sqrt{ } 5+i(-3-\sqrt{ } 5)$ & 2 & -2 & $1+\sqrt{ } 5+i(3+\sqrt{ } 5)$ & bed \\
\hline 28 & $-1-\sqrt{ } 5+i(-3-\sqrt{ } 5)$ & 2 & -2 & $-1-\sqrt{ } 5+i(3+\sqrt{ } 5)$ & bde \\
\hline 29 & $-3+\sqrt{ } 5+i(1-\sqrt{ } 5)$ & 2 & 2 & $3-\sqrt{ } 5+i(1-\sqrt{ } 5)$ & bec \\
\hline 30 & $-3+\sqrt{ } 5+i(-1+\sqrt{ } 5)$ & 2 & 2 & $3-\sqrt{ } 5+i(-1+\sqrt{ } 5)$ & bce \\
\hline 31 & $3-\sqrt{ } 5+i(-1+\sqrt{ } 5)$ & 2 & 2 & $-3+\sqrt{ } 5+i(-1+\sqrt{ } 5)$ & aed \\
\hline 32 & $3-\sqrt{ } 5+i(1-\sqrt{ } 5)$ & 2 & 2 & $-3+\sqrt{ } 5+i(1-\sqrt{ } 5)$ & ade \\
\hline 33 & 2 & $-1-\sqrt{ } 5+i(-1+\sqrt{ } 5)$ & $1+\sqrt{ } 5+i(-1+\sqrt{ } 5)$ & 2 & cde \\
\hline 34 & 2 & $1+\sqrt{ } 5+i(1-\sqrt{ } 5)$ & $-1-\sqrt{ } 5+i(1-\sqrt{ } 5)$ & 2 & ced \\
\hline 35 & 2 & $-1-\sqrt{ } 5+i(1-\sqrt{ } 5)$ & $1+\sqrt{ } 5+i(1-\sqrt{ } 5)$ & 2 & $a e b$ \\
\hline 36 & 2 & $1+\sqrt{ } 5+i(-1+\sqrt{ } 5)$ & $-1-\sqrt{ } 5+i(-1+\sqrt{ } 5)$ & 2 & $a b e$ \\
\hline 37 & $-1-\sqrt{ } 5+i(-3-\sqrt{ } 5)$ & 2 & 2 & $1+\sqrt{ } 5+i(-3-\sqrt{ } 5)$ & abede \\
\hline 38 & $-1-\sqrt{ } 5+i(1-\sqrt{ } 5)$ & 2 & 2 & $1+\sqrt{ } 5+i(1-\sqrt{ } 5)$ & $a c e b d$ \\
\hline 39 & $-1-\sqrt{ } 5+i(-1+\sqrt{ } 5)$ & 2 & 2 & $1+\sqrt{ } 5+i(-1+\sqrt{ } 5)$ & adbec \\
\hline 40 & $-1-\sqrt{ } 5+i(3+\sqrt{ } 5)$ & 2 & 2 & $1+\sqrt{ } 5+i(3+\sqrt{ } 5)$ & $a e d c b$ \\
\hline 41 & $1+\sqrt{ } 5+i(3+\sqrt{ } 5)$ & 2 & 2 & $-1-\sqrt{ } 5+i(3+\sqrt{ } 5)$ & $a d c e b$ \\
\hline 42 & $1+\sqrt{ } 5+i(-1+\sqrt{ } 5)$ & 2 & 2 & $-1-\sqrt{ } 5+i(-1+\sqrt{ } 5)$ & acbde \\
\hline 43 & $1+\sqrt{ } 5+i(1-\sqrt{ } 5)$ & 2 & 2 & $-1-\sqrt{ } 5+i(1-\sqrt{ } 5)$ & $a e d b c$ \\
\hline 44 & $1+\sqrt{ } 5+i(-3-\sqrt{ } 5)$ & 2 & 2 & $-1-\sqrt{ } 5+i(-3-\sqrt{ } 5)$ & abecd \\
\hline 45 & $-1-\sqrt{ } 5+i(-1+\sqrt{ } 5)$ & 2 & -2 & $-1-\sqrt{ } 5+i(1-\sqrt{ } 5)$ & acbed \\
\hline
\end{tabular}




\begin{tabular}{|r|r|r|r|r|l|}
46 & $-3+\sqrt{ } 5+i(-1+\sqrt{ } 5)$ & 2 & -2 & $-3+\sqrt{ } 5+i(1-\sqrt{ } 5)$ & abdce \\
47 & $3-\sqrt{ } 5+i(-1+\sqrt{ } 5)$ & 2 & -2 & $3-\sqrt{ } 5+i(1-\sqrt{ } 5)$ & aecdb \\
48 & $1+\sqrt{ } 5+i(-1+\sqrt{ } 5)$ & 2 & -2 & $1+\sqrt{ } 5+i(1-\sqrt{ } 5)$ & adebc \\
49 & $1+\sqrt{ } 5+i(1-\sqrt{ } 5)$ & 2 & -2 & $1+\sqrt{ } 5+i(-1+\sqrt{ } 5)$ & aecbd \\
50 & $3-\sqrt{ } 5+i(1-\sqrt{ } 5)$ & 2 & -2 & $3-\sqrt{ } 5+i(-1+\sqrt{ } 5)$ & acdeb \\
51 & $-3+\sqrt{ } 5+i(1-\sqrt{ } 5)$ & 2 & -2 & $-3+\sqrt{ } 5+i(-1+\sqrt{ } 5)$ & abedc \\
52 & $-1-\sqrt{ } 5+i(1-\sqrt{ } 5)$ & 2 & -2 & $-1-\sqrt{ } 5+i(-1+\sqrt{ } 5)$ & adbce \\
53 & 2 & $-3+\sqrt{ } 5+i(-1+\sqrt{ } 5)$ & $3-\sqrt{ } 5+i(-1+\sqrt{ } 5)$ & 2 & aebdc \\
54 & 2 & $-1-\sqrt{ } 5+i(3+\sqrt{ } 5)$ & $1+\sqrt{ } 5+i(3+\sqrt{ } 5)$ & 2 & abced \\
55 & 2 & $1+\sqrt{ } 5+i(-3-\sqrt{ } 5)$ & $-1-\sqrt{ } 5+i(-3-\sqrt{ } 5)$ & 2 & adecb \\
56 & 2 & $3-\sqrt{ } 5+i(1-\sqrt{ } 5)$ & $-3+\sqrt{ } 5+i(1-\sqrt{ } 5)$ & 2 & acdbe \\
57 & 2 & $-3+\sqrt{ } 5+i(1-\sqrt{ } 5)$ & $3-\sqrt{ } 5+i(1-\sqrt{ } 5)$ & 2 & abdec \\
58 & 2 & $-1-\sqrt{ } 5+i(-3-\sqrt{ } 5)$ & $1+\sqrt{ } 5+i(-3-\sqrt{ } 5)$ & 2 & adcbe \\
59 & 2 & $1+\sqrt{ } 5+i(3+\sqrt{ } 5)$ & $-1-\sqrt{ } 5+i(3+\sqrt{ } 5)$ & 2 & aebcd \\
60 & 2 & $3-\sqrt{ } 5+i(-1+\sqrt{ } 5)$ & $-3+\sqrt{ } 5+i(-1+\sqrt{ } 5)$ & 2 & acedb \\
& & &
\end{tabular}

107. Taking out of the foregoing group of 60 a group of 12 contained in it, viz. that corresponding to the positive substitutions of the four letters abcd, it is easy to see, that there is a transformation $(i, 0,0,1)$, that is, $s$ into is, which can be taken for the substitution $a d b c$, and also to complete thence the group of 24 . And we have thus the following Table.

Groups of 12 and 24. Pole at $\Theta$.

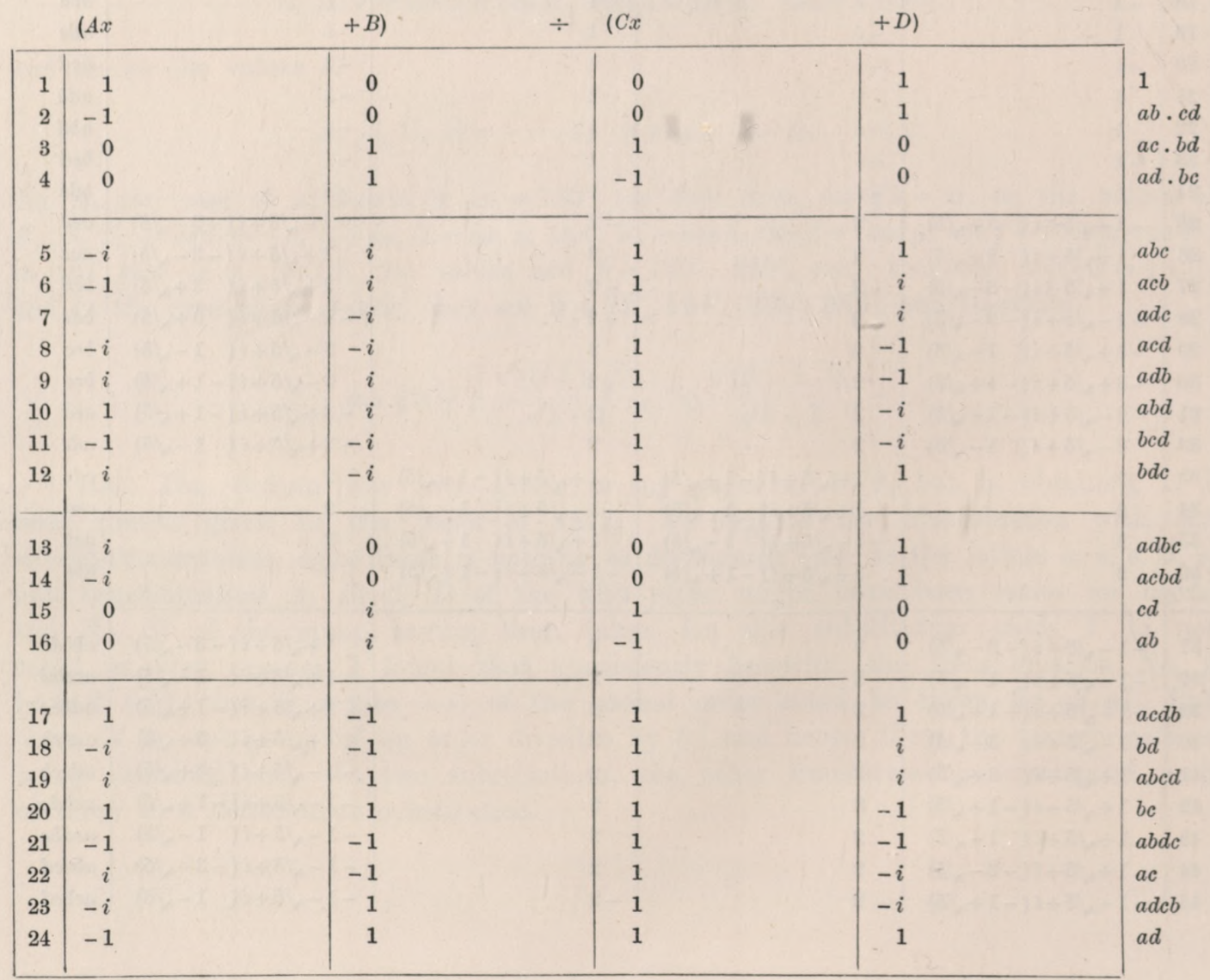


108. The group of 60 was obtained in the $A$-form by Gordan in his paper. The passage from the $\Theta$-form to the $A$-form is made as follows: let $X, Y, Z$ be the coordinates of a point when the axes are as in the $\Theta$-form, $X_{1}, Y_{1}, Z_{1}$ the coordinates of the same point when the axes are as in the $A$-form: we may write

where

$$
X, Y, Z=\mathrm{b} X_{1}-\mathrm{a} Z_{1}: Y_{1}: \mathrm{a} X_{1}+\mathrm{b} Z_{1},
$$

$$
\mathrm{a}, \mathrm{b}=\sqrt{\frac{5-\sqrt{5}}{10}}, \sqrt{\frac{5+\sqrt{ } 5}{10}}
$$

then, if the equations of an axis of rotation referred to the first set of coordinates are $X: Y: Z=L: M: N$, those of the same axis referred to the second set of coordinates are

or taking these to be

$$
\mathrm{b} X_{1}+\mathrm{a} Z_{1}: Y_{1}:-\mathrm{a} X_{1}+\mathrm{b} Z_{1}=L: M: N ;
$$

we may write

$$
X_{1}: Y_{1}: Z_{1}=L_{1}: M_{1}: N_{1} \text {, }
$$

$$
L_{1}, M_{1}, N_{1}=\mathrm{b} L+\mathrm{a} N, M,-\mathrm{a} L+\mathrm{b} N:
$$

these values are such that

$$
L_{1}^{2}+M_{1}^{2}+N_{1}^{2}=L^{2}+M^{2}+N^{2},
$$

and hence, $\lambda, \mu, \nu$ and $\lambda_{1}, \mu_{1}, \nu_{1}$ being the rotations, we may write

$$
L, M, N=9 \lambda, 9 \mu, 9 \nu ; \quad L_{1}, M_{1}, N_{1}=9 \lambda_{1}, 9 \mu_{1}, 9 \nu_{1} ;
$$

where 9 has the same value in each set of equations. From the equations

we have

$$
A: B: C: D=-\nu-i: \lambda+i \mu: \lambda-i \mu: \nu-i,
$$

and similarly

$$
B+C: B-C: D-A: D+A=\lambda: i \mu: \nu:-i
$$

$$
=L: i M: N:-i 9 \text {, }
$$

$$
B_{1}+C_{1}: B_{1}-C_{1}: D_{1}-A_{1}: D_{1}+A_{1}=L_{1}: i M_{1}: N_{1}:-i 9 \text {. }
$$

Hence we may write

$$
\begin{aligned}
& B_{1}+C_{1}=\mathrm{b}(B+C)+\mathrm{a}(D-A), \\
& B_{1}-C_{1}=\quad B-C \\
& D_{1}-A_{1}=-\mathrm{a}(B+C)+\mathrm{b}(D-A), \\
& D_{1}+A_{1}=\quad D+A ;
\end{aligned}
$$

or say,

$$
\begin{aligned}
& A_{1}=\mathrm{a}(B+C)-\mathrm{b}(D-A)+(D+A), \\
& B_{1}=\mathrm{b}(B+C)+\mathrm{a}(D-A)+(B-C), \\
& C_{1}=\mathrm{b}(B+C)+\mathrm{a}(D-A)-(B-C), \\
& D_{1}=-\mathrm{a}(B+C)+\mathrm{b}(D-A)+(D+A),
\end{aligned}
$$

which are the values for a transformation $\left(A_{1}, B_{1}, C_{1}, D_{1}\right)$ in the $A$-form: of course, as only the ratios are material, the values may be multiplied by any common factor. 
109. The results are exhibited in terms of $\epsilon$, an imaginary fifth root of unity: taking $\epsilon=\cos 72^{\circ}+i \sin 72^{\circ}$, we have

$$
\begin{gathered}
\epsilon, \epsilon^{4}=\frac{\sqrt{ } 5-1}{4} \pm i \sqrt{\frac{5+\sqrt{ } 5}{8}} \\
\epsilon^{2}, \epsilon^{3}=-\frac{\sqrt{ } 5+1}{4} \pm i \sqrt{\frac{5-\sqrt{ } 5}{8}}
\end{gathered}
$$

where the upper signs belong to $\epsilon, \epsilon^{2}$ and the lower to $\epsilon^{4}, \epsilon^{3}$. It may be remarked that

$$
\frac{1}{\mathrm{a}}=\sqrt{\frac{5+\sqrt{ } 5}{2}}, \quad \frac{1}{\mathrm{~b}}=\sqrt{\frac{5-\sqrt{ } 5}{2}}, \quad \frac{\mathrm{b}}{\mathrm{a}}=\frac{\sqrt{ } 5+1}{2}, \quad \frac{\mathrm{a}}{\mathrm{b}}=\frac{\sqrt{ } 5-1}{2} .
$$

For instance, we have in the $\Theta$-group $(A, B, C, D)=(-1,0,0,1) ; a b . c d$ : and thence in the $A$-group $A_{1}, B_{1}, C_{1}, D_{1}=(-2 \mathrm{~b}, 2 \mathrm{a}, 2 \mathrm{a}, 2 \mathrm{~b}) ; a b . c d$ : or say this is

$$
\left(-1, \frac{\mathrm{a}}{\mathrm{b}}, \frac{\mathrm{a}}{\mathrm{b}}, 1\right),=\left(-1, \epsilon+\epsilon^{4}, \epsilon+\epsilon^{4}, 1\right)
$$

which in the Table is given as $\left(-\epsilon^{3}, \epsilon^{2}+\epsilon^{4}, \epsilon^{2}+\epsilon^{4}, \epsilon^{3}\right) ; a b . c d$.

By effecting the passage to the $A$-group in this manner, we of course obtain the proper substitution corresponding to each transformation: but I found it easier starting from two transformations and the corresponding substitutions, to obtain thence by successive compositions the entire group.

110. Homographic Transformations. The group of 60 . Pole at $A$.
$\Theta$ No. $(A s$

\begin{tabular}{|c|c|c|c|c|c|c|}
\hline 1 & 1 & 1 & & & 1 & 1 \\
\hline 2 & 4 & 0 & -1 & 1 & 0 & $a d . b c$ \\
\hline 3 & 13 & 0 & $-\epsilon^{4}$ & 1 & 0 & $a c . b e$ \\
\hline 4 & 9 & 0 & $-\epsilon^{3}$ & 1 & 0 & ae.cd \\
\hline 5 & 10 & 0 & $-\epsilon^{2}$ & 1 & 0 & $a b . d e$ \\
\hline 6 & 14 & 0 & $-\epsilon$ & 1 & 0 & $b d . c e$ \\
\hline 7 & 6 & $\epsilon+\epsilon^{2}$ & $\epsilon^{4}$ & 1 & $-\left(\epsilon+\epsilon^{3}\right)$ & $a e . b c$ \\
\hline 8 & 5 & $\epsilon+\epsilon^{3}$ & 1 & $\epsilon^{4}$ & $-\left(\epsilon+\epsilon^{3}\right)$ & $b c . d e$ \\
\hline 9 & 16 & $\epsilon+\epsilon^{3}$ & $\epsilon$ & $\epsilon^{3}$ & $-\left(\epsilon+\epsilon^{3}\right)$ & $a c \cdot d e$ \\
\hline 10 & 3 & $\epsilon+\epsilon^{3}$ & $\epsilon^{2}$ & $\epsilon^{2}$ & $-\left(\epsilon+\epsilon^{3}\right)$ & $a c . b d$ \\
\hline 11 & 15 & $\epsilon+\epsilon^{3}$ & $\epsilon^{3}$ & $\epsilon$ & $-\left(\epsilon+\epsilon^{3}\right)$ & $a e . b d$ \\
\hline 12 & 12 & -1 & $\epsilon+\epsilon^{3}$ & $\epsilon^{2}+\epsilon^{4}$ & 1 & $a b . c e$ \\
\hline 13 & 11 & $-\epsilon$ & $\epsilon^{3}+1$ & $\epsilon^{2}+\epsilon^{4}$ & $\epsilon$ & $b e . c d$ \\
\hline 14 & 7 & $-\epsilon^{2}$ & $1+\epsilon^{2}$ & $\epsilon^{2}+\epsilon^{4}$ & $\epsilon^{2}$ & ad.ce \\
\hline 15 & 2 & $-\epsilon^{3}$ & $\epsilon^{2}+\epsilon^{4}$ & $\epsilon^{2}+\epsilon^{4}$ & $\epsilon^{3}$ & $a b \cdot c d$ \\
\hline 16 & 8 & $-\epsilon^{4}$ & $\epsilon^{4}+\epsilon$ & $\epsilon^{2}+\epsilon^{4}$ & $\epsilon^{4}$ & $a d . b e$ \\
\hline
\end{tabular}
$+B)$
$\div(\mathrm{Cs}$
$+D$ ) 


\begin{tabular}{|c|c|c|c|c|c|c|}
\hline 17 & 21 & $\epsilon^{3}+1$ & $\epsilon$ & 1 & $-\left(\epsilon+\epsilon^{3}\right)$ & $a d b$ \\
\hline 18 & 35 & $\epsilon^{3}+1$ & $\epsilon^{2}$ & $\epsilon^{4}$ & $-\left(\epsilon+\epsilon^{3}\right)$ & $a e b$ \\
\hline 19 & 30 & $\epsilon^{3}+1$ & $\epsilon^{3}$ & $\epsilon^{3}$ & $-\left(\epsilon+\epsilon^{3}\right)$ & bce \\
\hline 20 & 34 & $\epsilon^{3}+1$ & $\epsilon^{4}$ & $\epsilon^{2}$ & $-\left(\epsilon+\epsilon^{3}\right)$ & ced \\
\hline 21 & 19 & $\epsilon^{3}+1$ & 1 & $\epsilon$ & $-\left(\epsilon+\epsilon^{3}\right)$ & $a d c$ \\
\hline 22 & 33 & $\epsilon+\epsilon^{4}$ & $\epsilon^{2}$ & 1 & $-\left(\epsilon+\epsilon^{3}\right)$ & cde \\
\hline 23 & 20 & $\epsilon+\epsilon^{4}$ & $\epsilon^{3}$ & $\epsilon^{4}$ & $-\left(\epsilon+\epsilon^{3}\right)$ & acd \\
\hline 24 & 22 & $\epsilon+\epsilon^{4}$ & $\epsilon^{4}$ & $\epsilon^{3}$ & $-\left(\epsilon+\epsilon^{3}\right)$ & $a b d$ \\
\hline 25 & 36 & $\epsilon+\epsilon^{4}$ & 1 & $\epsilon^{2}$ & $-\left(\epsilon+\epsilon^{3}\right)$ & $a b e$ \\
\hline 26 & 29 & $\epsilon+\epsilon^{4}$ & $\epsilon$ & $\epsilon$ & $-\left(\epsilon+\epsilon^{3}\right)$ & bec \\
\hline 27 & 31 & $-\epsilon$ & $\epsilon^{2}+\epsilon^{4}$ & $\epsilon^{2}+\epsilon^{4}$ & 1 & aed \\
\hline 28 & 17 & $-\epsilon^{2}$ & $\epsilon^{4}+\epsilon$ & $\epsilon^{2}+\epsilon^{4}$ & $\epsilon$ & $a b c$ \\
\hline 29 & 27 & $-\epsilon^{3}$ & $\epsilon+\epsilon^{3}$ & $\epsilon^{2}+\epsilon^{4}$ & $\epsilon^{2}$ & bed \\
\hline 30 & 25 & $-\epsilon^{4}$ & $\epsilon^{3}+1$ & $\epsilon^{2}+\epsilon^{4}$ & $\epsilon^{3}$ & $a e c$ \\
\hline 31 & 23 & -1 & $1+\epsilon^{2}$ & $\epsilon^{2}+\epsilon^{4}$ & $\epsilon^{4}$ & $b e d$ \\
\hline 32 & 24 & $-\epsilon^{4}$ & $1+\epsilon^{2}$ & $\epsilon^{2}+\epsilon^{4}$ & 1 & $b d c$ \\
\hline 33 & 32 & -1 & $\epsilon^{2}+\epsilon^{4}$ & $\epsilon^{2}+\epsilon^{4}$ & $\epsilon$ & ade \\
\hline 34 & 18 & $-\epsilon$ & $\epsilon^{4}+\epsilon$ & $\epsilon^{2}+\epsilon^{4}$ & $\epsilon^{2}$ & $a c b$ \\
\hline 35 & 28 & $-\epsilon^{2}$ & $\epsilon+\epsilon^{3}$ & $\epsilon^{2}+\epsilon^{4}$ & $\epsilon^{3}$ & bde \\
\hline 36 & 26 & $-\epsilon^{3}$ & $\epsilon^{3}+1$ & $\epsilon^{2}+\epsilon^{4}$ & $\epsilon^{4}$ & ace \\
\hline 37 & 44 & $\epsilon$ & 0 & 0 & 1 & abecd \\
\hline 38 & 43 & $\epsilon^{2}$ & 0 & 0 & 1 & $a e d b c$ \\
\hline 39 & 42 & $\epsilon^{3}$ & 0 & 0 & 1 & acbde \\
\hline 40 & 41 & $\epsilon^{4}$ & 0 & 0 & 1 & adceb \\
\hline 41 & 38 & $\epsilon^{2}+\epsilon^{4}$ & 1 & 1 & $-\left(\epsilon+\epsilon^{3}\right)$ & acebd \\
\hline 42 & 46 & $\epsilon^{2}+\epsilon^{4}$ & $\epsilon$ & $\epsilon^{4}$ & $-\left(\epsilon+\epsilon^{3}\right)$ & abdce \\
\hline 43 & 58 & $\epsilon^{2}+\epsilon^{4}$ & $\epsilon^{2}$ & $\epsilon^{3}$ & $-\left(\epsilon+\epsilon^{3}\right)$ & adcbe \\
\hline 44 & 55 & $\epsilon^{2}+\epsilon^{4}$ & $\epsilon^{3}$ & $\epsilon^{2}$ & $-\left(\epsilon+\epsilon^{3}\right)$ & adecb \\
\hline 45 & 50 & $\epsilon^{2}+\epsilon^{4}$ & $\epsilon^{4}$ & $\epsilon$ & $-\left(\epsilon+\epsilon^{3}\right)$ & $a c d e b$ \\
\hline 46 & 51 & $1+\epsilon^{2}$ & $\epsilon^{3}$ & 1 & $-\left(\epsilon+\epsilon^{3}\right)$ & $a b e d c$ \\
\hline 47 & 39 & $1+\epsilon^{2}$ & $\epsilon^{4}$ & $\epsilon^{4}$ & $-\left(\epsilon+\epsilon^{3}\right)$ & adbec \\
\hline 48 & 47 & $1+\epsilon^{2}$ & 1 & $\epsilon^{3}$ & $-\left(\epsilon+\epsilon^{3}\right)$ & $a e c d b$ \\
\hline 49 & 59 & $1+\epsilon^{2}$ & $\epsilon$ & $\epsilon^{2}$ & $-\left(\epsilon+\epsilon^{3}\right)$ & aebcd \\
\hline 50 & 54 & $1+\epsilon^{2}$ & $\epsilon^{2}$ & $\epsilon$ & $-\left(\epsilon+\epsilon^{3}\right)$ & abced \\
\hline 51 & 56 & $-\epsilon^{2}$ & $\epsilon^{3}+1$ & $\epsilon^{2}+\epsilon^{4}$ & 1 & acdbe \\
\hline 52 & 49 & $-\epsilon^{3}$ & $1+\epsilon^{2}$ & $\epsilon^{2}+\epsilon^{4}$ & $\epsilon$ & $a e c b d$ \\
\hline 53 & 37 & $-\epsilon^{4}$ & $\epsilon^{2}+\epsilon^{4}$ & $\epsilon^{2}+\epsilon^{4}$ & $\epsilon^{2}$ & abcde \\
\hline 54 & 45 & -1 & $\epsilon^{4}+\epsilon$ & $\epsilon^{2}+\epsilon^{4}$ & $\epsilon^{3}$ & acbed \\
\hline 55 & 57 & $-\epsilon$ & $\epsilon+\epsilon^{3}$ & $\epsilon^{2}+\epsilon^{4}$ & $\epsilon^{4}$ & abdec \\
\hline 56 & 48 & $-\epsilon^{3}$ & $\epsilon^{4}+\epsilon$ & $\epsilon^{2}+\epsilon^{4}$ & 1 & adebc \\
\hline 57 & 60 & $-\epsilon^{4}$ & $\epsilon+\epsilon^{3}$ & $\epsilon^{2}+\epsilon^{4}$ & $\epsilon$ & $a c e d b$ \\
\hline 58 & 53 & -1 & $\epsilon^{3}+1$ & $\epsilon^{2}+\epsilon^{4}$ & $\epsilon^{2}$ & $a e b d c$ \\
\hline 59 & 52 & $-\epsilon$ & $1+\epsilon^{2}$ & $\epsilon^{2}+\epsilon^{4}$ & $\epsilon^{3}$ & adbce \\
\hline 60 & 40 & $-\epsilon^{2}$ & $\epsilon^{2}+\epsilon^{4}$ & $\epsilon^{2}+\epsilon^{4}$ & $\epsilon^{4}$ & $a e d c b$ \\
\hline
\end{tabular}

C. XI. 
111. Selecting the transformations which correspond to the positive substitutions $a b c d$, and completing the group of 24 we have

Homographic Transformations. The groups of 12 and 24. Pole at $A$.

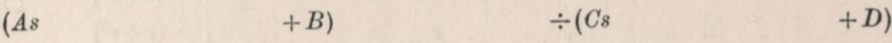

\begin{tabular}{|c|c|c|c|c|c|}
\hline 1 & 1 & 0 & 0 & 1 & 1 \\
\hline 2 & 0 & -1 & 1 & 0 & $a d \cdot b c$ \\
\hline 3 & $\epsilon+\epsilon^{3}$ & $\epsilon^{2}$ & $\epsilon^{2}$ & $-\left(\epsilon+\epsilon^{3}\right)$ & $a c . b d$ \\
\hline 4 & $-\epsilon^{3}$ & $\epsilon^{2}+\epsilon^{4}$ & $\epsilon^{2}+\epsilon^{4}$ & $\epsilon^{3}$ & $a b . c d$ \\
\hline 5 & $-\epsilon^{2}$ & $\epsilon+\epsilon^{4}$ & $\epsilon^{2}+\epsilon^{4}$ & $\epsilon$ & $a b c$ \\
\hline 6 & $-\epsilon$ & $\epsilon+\epsilon^{4}$ & $\epsilon^{2}+\epsilon^{4}$ & $\epsilon^{2}$ & $a c b$ \\
\hline 7 & $\epsilon+\epsilon^{4}$ & $\epsilon^{3}$ & $\epsilon^{4}$ & $-\left(\epsilon+\epsilon^{3}\right)$ & acd \\
\hline 8 & $\epsilon^{3}+1$ & 1 & $\epsilon$ & $-\left(\epsilon+\epsilon^{3}\right)$ & $a d c$ \\
\hline 9 & $\epsilon+\epsilon^{4}$ & $\epsilon^{4}$ & $\epsilon^{3}$ & $-\left(\epsilon+\epsilon^{3}\right)$ & $a b d$ \\
\hline 10 & $\epsilon^{3}+1$ & $\epsilon$ & & $-\left(\epsilon+\epsilon^{3}\right)$ & $a d b$ \\
\hline 11 & -1 & $1+\epsilon^{2}$ & $\epsilon^{2}+\epsilon^{4}$ & $\epsilon^{4}$ & $b c d$ \\
\hline 12 & $-\epsilon^{4}$ & $1+\epsilon^{2}$ & $\epsilon^{2}+\epsilon^{4}$ & 1 & $b d c$ \\
\hline 13 & 1 & $1+2 \epsilon^{4}$ & $1+2 \epsilon$ & -1 & $a b$ \\
\hline 14 & $-\epsilon^{2}+\epsilon^{3}$ & $1+\epsilon+3 \epsilon^{4}$ & $-1-3 \epsilon-\epsilon^{4}$ & $\epsilon^{2}-\epsilon^{3}$ & $c d$ \\
\hline 15 & $\epsilon^{2}-\epsilon^{4}$ & $3+\epsilon+\epsilon^{3}$ & $-1-3 \epsilon-\epsilon^{3}$ & $-\epsilon^{2}+\epsilon^{4}$ & $a c$ \\
\hline 16 & $-1+\epsilon^{2}$ & $-1-\epsilon^{2}+2 \epsilon^{4}$ & $1+\epsilon^{2}-2 \epsilon^{3}$ & $1-\epsilon^{2}$ & $b d$ \\
\hline 17 & $2+\epsilon^{3}+2 \epsilon^{4}$ & $-2-2 \epsilon^{2}-\epsilon^{3}$ & $2 \epsilon+\epsilon^{3}+2 \epsilon^{4}$ & $2 \epsilon+2 \epsilon^{2}+\epsilon^{3}$ & $a d$ \\
\hline 18 & $2+2 \epsilon^{2}+\epsilon^{3}$ & $2+\epsilon^{3}+2 \epsilon^{4}$ & $-2 \epsilon-2 \epsilon^{2}-\epsilon^{3}$ & $2 \epsilon+\epsilon^{3}+2 \epsilon^{4}$ & $b c$ \\
\hline 19 & $-2+\epsilon+\epsilon^{3}$ & $-\epsilon+\epsilon^{3}$ & $-\epsilon+\epsilon^{3}$ & $\epsilon+\epsilon^{3}-2 \epsilon^{4}$ & abed \\
\hline 20 & 1 & -1 & 1 & 1 & $a b d c$ \\
\hline 21 & 1 & 1 & -1 & 1 & $a c d b$ \\
\hline 22 & $1+\epsilon+3 \epsilon^{4}$ & $\epsilon^{2}-\epsilon^{3}$ & $\epsilon^{2}-\epsilon^{3}$ & $1+3 \epsilon+\epsilon^{4}$ & $a c b d$ \\
\hline 23 & $1+2 \epsilon^{4}$ & & & $-1-2 \epsilon$ & $a d b c$ \\
\hline 24 & $3+\epsilon+\epsilon^{3}$ & $-\epsilon^{2}+\epsilon^{4}$ & $-\epsilon^{2}+\epsilon^{4}$ & $1+3 \epsilon+\epsilon^{3}$ & $a d c b$ \\
\hline
\end{tabular}

As an example of the calculation we have $(A, B, C, D)=(0, i,-1,0) ; a b$. Hence

$$
\begin{aligned}
A_{1}, B_{1}, C_{1}, D_{1} & =(\mathrm{a}(i-1), \mathrm{b}(i-1)+i+1, \mathrm{~b}(i-1)-(i+1),-\mathrm{a}(i+1)), \\
& =\left(1, \frac{\mathrm{b}-i}{\mathrm{a}}, \frac{\mathrm{b}+i}{\mathrm{a}},-1\right) .
\end{aligned}
$$

The second and third coefficients are

$$
\frac{\sqrt{ } 5+1}{2}-i \sqrt{\frac{5+\sqrt{ } 5}{2}}, \frac{\sqrt{ } 5+1}{2}+i \sqrt{\frac{5+\sqrt{ } 5}{2}},
$$

which, in virtue of the values of $\epsilon$ and $\epsilon^{4}$, are $=1+2 \epsilon^{4}$ and $1+2 \epsilon$ respectively: or the result is as above $\left(1,1+2 \epsilon^{4}, 1+2 \epsilon,-1\right)$. 
112. In like manner for the passage from the $\Theta$-form to the $B$-form, if $X, Y, Z$ be the coordinates of a point on the spherical surface in regard to the $\Theta$-axes, $X_{2}, Y_{2}, Z_{2}$ those of the same point in regard to the $B$-axes, we may write

where

$$
X: Y: Z=X_{2}: \mathrm{b} Y_{2}+\mathrm{a} Z_{2}:-\mathrm{a} Y_{2}+\mathrm{b} Z_{2},
$$

$$
\mathrm{a}, \mathrm{b}=\frac{\sqrt{ } 5-1}{2 \sqrt{ } 3}, \frac{\sqrt{ } 5+1}{2 \sqrt{ } 3} .
$$

Hence $X: Y: Z=L: M: N$, being the equations of an axis of rotation in the first set of coordinates, those of the same axis in the second set of coordinates will be

or calling these

$$
X_{2}: \mathrm{b} Y_{2}+\mathrm{a} Z_{2}:-\mathrm{a} Y_{2}+\mathrm{b} Z_{2}=L: M: N,
$$

we have

$$
X_{2}: Y_{2}: Z_{2}=L_{2}: M_{2}: N_{2}
$$

$$
L_{2}, M_{2}, N_{2}=L: \mathrm{b} M-\mathrm{a} N: \mathrm{a} M+\mathrm{b} N:
$$

these values are such that

$$
L_{2}{ }^{2}+M_{2}{ }^{2}+N_{2}{ }^{2}=L^{2}+M^{2}+N^{2}
$$

or $\lambda, \mu, \nu, \lambda_{2}, \mu_{2}, \nu_{2}$ being the rotations, we have

$$
L, M, N=9 \lambda, 9 \mu, 9 \nu ; L_{2}, M_{2}, N_{2}=9 \lambda_{2}, 9 \mu_{2}, 9 \nu_{2},
$$

where 9 has the same value in the two sets of equations. We have thus

$$
\begin{aligned}
& B+C: B-C: D-A: D+A=L: 2 M: N:-i \mathrm{~A}, \\
& B_{2}+C_{2}: B_{2}-C_{2}: D_{2}-A_{2}: D_{2}+A_{2}=L_{2}: 2 M_{2}: N_{2}:-i \mathrm{~A}
\end{aligned}
$$

and hence

and thence

$$
\begin{aligned}
& B_{2}+C_{2}=\quad B+C \\
& B_{2}-C_{2}=\quad \mathrm{b}(B-C)-\mathrm{a} i(D-A) \\
& D_{2}-A_{2}=-\mathrm{a} i(B-C)+\mathrm{b}(D-A) \\
& D_{2}+A_{2}=\quad D+A ;
\end{aligned}
$$

$$
\begin{aligned}
& A_{2}=\mathrm{a} i(B-C)-\mathrm{b}(D-A)+(D+A), \\
& B_{2}=\mathrm{b}(B-C)-\mathrm{a} i(D-A)+(B+C), \\
& C_{2}=-\mathrm{b}(B-C)+\mathrm{a} i(D-A)+(B+C), \\
& D_{2}=-\mathrm{a} i(B-C)+\mathrm{b}(D-A)+(D+A) .
\end{aligned}
$$

113. As an example of the transformation, take then

$$
(A, B, C, D)=(2,-3+\sqrt{ } 5+i(1-\sqrt{ } 5),-3+\sqrt{ } 5+i(-1+\sqrt{ } 5),-2) \quad[b c . d e]:
$$

$$
B-C, B+C, D-A, D+A=i(1-\sqrt{ } 5),-3+\sqrt{ } 5,-2,0
$$


and thence

$$
\begin{aligned}
& A_{2}=\frac{1}{2 \sqrt{ } 3}(6-2 \sqrt{ } 5)+\frac{1}{2 \sqrt{ } 3}(2+2 \sqrt{ } 5), \\
& B_{2}=\frac{1}{2 \sqrt{ } 3}(-4 i)+\frac{1}{2 \sqrt{ } 3}(2 i(1+\sqrt{ } 5)+(-3+\sqrt{ } 5)), \\
& C_{2}=\frac{1}{2 \sqrt{ } 3}(4 i)+\frac{1}{2 \sqrt{ } 3}(2 i(1-\sqrt{ } 5)+(-3+\sqrt{ } 5)), \\
& D_{2}=\frac{1}{2 \sqrt{ } 3}(-6+2 \sqrt{ } 5)+\frac{1}{2 \sqrt{ } 3}(-2-2 \sqrt{ } 5) ;
\end{aligned}
$$

viz. multiplying by $2 \sqrt{ } 3$, these are

$$
8, \quad i(-6+2 \sqrt{ } 5)+2 \sqrt{ } 3(-3+\sqrt{ } 5), \quad i(6-2 \sqrt{ } 5)+2 \sqrt{ } 3(-3+\sqrt{ } 5), \quad-8,
$$

that is,

$$
8, \quad(-6+2 \sqrt{ } 5)(i+\sqrt{ } 3), \quad(-6+2 \sqrt{ } 5)(-i+\sqrt{ } 3), \quad-8,
$$

or since

dividing by 4 these are

\begin{tabular}{|c|c|c|c|c|c|}
\hline & (As & $+B)$ & $\div(C s$ & $+D)$ & \\
\hline 1 & 1 & 0 & 0 & 1 & 1 \\
\hline 2 & 0 & 1 & 1 & 0 & $a c, b d$ \\
\hline 3 & 0 & $\omega$ & 1 & 0 & $a e \cdot b d$ \\
\hline 4 & 0 & $\omega^{2}$ & 1 & 0 & $b d . c e$ \\
\hline 5 & 2 & $i(3-\sqrt{ } 5)$ & $i(-3+\sqrt{ } 5)$ & -2 & $a b \cdot c d$ \\
\hline 6 & 2 & $i(-3-\sqrt{ } 5)$ & $i(\quad 3+\sqrt{ } 5)$ & -2 & $a d . b c$ \\
\hline 7 & 2 & $i(3-\sqrt{ } 5) \omega$ & $i(-3+\sqrt{ } 5) \omega^{2}$ & -2 & $b c \cdot d e$ \\
\hline 8 & 2 & $i(-3-\sqrt{ } 5) \omega$ & $i(\quad 3+\sqrt{ } 5) \omega^{2}$ & -2 & be.cd \\
\hline 9 & 2 & $i(3-\sqrt{ } 5) \omega^{2}$ & $i(\quad-3+\sqrt{ } 5) \omega$ & -2 & $a d . b e$ \\
\hline 10 & 2 & $i(-3-\sqrt{ } 5) \omega^{2}$ & $i(\quad 3+\sqrt{ } 5) \omega$ & -2 & $a b . d e$ \\
\hline 11 & 2 & $(-\sqrt{ } 3-i \sqrt{ } 5) \omega$ & $(-\sqrt{ } 3+i \sqrt{ } 5) \omega^{2}$ & -2 & $a b . c e$ \\
\hline 12 & 2 & $-\sqrt{ } 3-i \sqrt{ } 5$ & $-\sqrt{ } 3+i \sqrt{ } 5$ & -2 & $a c \cdot b e$ \\
\hline 13 & 2 & $(-\sqrt{ } 3-i \sqrt{ } 5) \omega^{2}$ & $(-\sqrt{ } 3+i \sqrt{ } 5) \omega$ & -2 & $a e \cdot b c$ \\
\hline 14 & 2 & $\sqrt{ } 3-i \sqrt{ } 5$ & $\sqrt{ } 3+i \sqrt{ } 5$ & -2 & $a c \cdot d e$ \\
\hline 15 & 2 & $(\sqrt{ } 3-i \sqrt{ } 5) \omega$ & $(\sqrt{ } 3+i \sqrt{ } 5) \omega^{2}$ & -2 & ad.ce \\
\hline 16 & 2 & $(\sqrt{ } 3-i \sqrt{ } 5) \omega^{2}$ & $(\sqrt{ } 3+i \sqrt{ } 5) \omega$ & -2 & $a e \cdot c d$ \\
\hline
\end{tabular}

$$
2+\sqrt{ } 3=-2 i \omega \text { and }-2+\sqrt{ } 3=2 i \omega^{2}
$$

as in the table.

$$
2, \quad i(3-\sqrt{ } 5) \omega, \quad i(-3+\sqrt{ } 5) \omega^{2}, \quad-2,
$$

114. Homographic Transformations. The group of 60 . Pole at $B$.

$$
\omega=\frac{1}{2}\left(-1+i_{\wedge} / 3\right) \text {. }
$$




\begin{tabular}{|c|c|c|c|c|c|}
\hline 17 & $\omega$ & 0 & 0 & 1 & ace \\
\hline 18 & $\omega^{2}$ & 0 & 0 & 1 & $a e c$ \\
\hline 19 & $\sqrt{ } 3-i \sqrt{ } 5$ & 2 & -2 & $\sqrt{ } 3+i \sqrt{ } 5$ & bed \\
\hline 20 & $-\sqrt{ } 3-i \sqrt{ } 5$ & 2 & -2 & $-\sqrt{ } 3+i \sqrt{ } 5$ & $b d e$ \\
\hline 21 & $-\sqrt{ } 3-i \sqrt{ } 5$ & $2 \omega^{2}$ & $-2 \omega$ & $-\sqrt{ } 3+i \sqrt{ } 5$ & $b d c$ \\
\hline 22 & $\sqrt{ } 3-i \sqrt{ } 5$ & $2 \omega^{2}$ & $-2 \omega$ & $\sqrt{ } 3+i \sqrt{ } 5$ & $b c d$ \\
\hline 23 & $-\sqrt{ } 3-i \sqrt{ } 5$ & $2 \omega$ & $-2 \omega^{2}$ & $-\sqrt{ } 3+i \sqrt{ } 5$ & $a b d$ \\
\hline 24 & $\sqrt{ } 3-i \sqrt{ } 5$ & $2 \omega$ & $-2 \omega^{2}$ & $\sqrt{ } 3+i \sqrt{ } 5$ & $a d b$ \\
\hline 25 & $2 \omega^{2}$ & $-\sqrt{ } 3-i \sqrt{ } 5$ & $-\sqrt{ } 3+i \sqrt{ } 5$ & $-2 \omega$ & $a b c$ \\
\hline 26 & $2 \omega$ & $-\sqrt{ } 3-i \sqrt{ } 5$ & $-\sqrt{ } 3+i \sqrt{ } 5$ & $-2 \omega^{2}$ & $a c b$ \\
\hline 27 & $2 \omega^{2}$ & $-\sqrt{ } 3-i \sqrt{ } 5$ & $(-\sqrt{ } 3+i \sqrt{ } 5) \omega^{2}$ & -2 & abe \\
\hline 28 & 2 & $-\sqrt{ } 3-i \sqrt{ } 5$ & $(-\sqrt{ } 3+i \sqrt{ } 5) \omega^{2}$ & $-2 \omega^{2}$ & $a e b$ \\
\hline 29 & $2 \omega$ & $\sqrt{ } 3-i \sqrt{ } 5$ & $\sqrt{ } 3+i \sqrt{ } 5$ & $-2 \omega^{2}$ & acd \\
\hline 30 & $2 \omega^{2}$ & $\sqrt{ } 3-i \sqrt{ } 5$ & $\sqrt{ } 3+i \sqrt{ } 5$ & $-2 \omega$ & $a d c$ \\
\hline 31 & $2 \omega^{2}$ & $\sqrt{ } 3-i \sqrt{ } 5$ & $(\sqrt{ } 3+i \sqrt{ } 5) \omega^{2}$ & -2 & ade \\
\hline 32 & 2 & $\sqrt{ } 3-i \sqrt{ } 5$ & $(\sqrt{ } 3+i \sqrt{ } 5) \omega^{2}$ & $-2 \omega^{2}$ & aed \\
\hline 33 & 2 & $-\sqrt{ } 3-i \sqrt{ } / 5$ & $(-\sqrt{ } 3+i \sqrt{ } 5) \omega$ & $-2 \omega$ & bce \\
\hline 34 & $2 \omega$ & $-\sqrt{ } 3-i \sqrt{ } 5$ & $(-\sqrt{ } 3+i \sqrt{ } 5) \omega$ & -2 & $b e c$ \\
\hline 35 & $2 \omega$ & $\sqrt{ } 3-i \sqrt{ } 5$ & $(\sqrt{ } 3+i \sqrt{ } 5) \omega$ & -2 & $c d e$ \\
\hline 36 & 2 & $\sqrt{ } 3-i \sqrt{ } 5$ & $(\sqrt{ } 3+i \sqrt{ } 5) \omega$ & $-2 \omega$ & ced \\
\hline 37 & 2 & $i(3-\sqrt{ } 5) \omega^{2}$ & $i(-3+\sqrt{ } 5)$ & $-2 \omega^{2}$ & $a d c e b$ \\
\hline 38 & $-\sqrt{ } 3-i \sqrt{ } 5$ & $+2 \omega^{2}$ & -2 & $(-\sqrt{ } 3+i \sqrt{ } 5) \omega^{2}$ & acbde \\
\hline 39 & $\sqrt{ } 3-i \sqrt{ } 5$ & 2 & $-2 \omega$ & $(\sqrt{ } 3+i \sqrt{ } 5) \omega$ & $a e d b c$ \\
\hline 40 & 2 & $i(3-\sqrt{ } 5)$ & $i(-3+\sqrt{ } 5) \omega$ & $-2 \omega$ & abecd \\
\hline 41 & 2 & $i(3-\sqrt{ } 5) \omega$ & $i(-3+\sqrt{ } 5)$ & $-2 \omega$ & $a e d c b$ \\
\hline 42 & $-\sqrt{ } 3-i \sqrt{ } 5$ & $2 \omega$ & -2 & $(-\sqrt{ } 3+i \sqrt{ } 5) \omega$ & adbec \\
\hline 43 & $\sqrt{ } 3-i \sqrt{ } 5$ & 2 & $-2 \omega^{2}$ & $(\sqrt{ } 3+i \sqrt{ } 5) \omega^{2}$ & acebd \\
\hline 44 & 2 & $i(3-\sqrt{ } 5)$ & $i(-3+\sqrt{ } 5) \omega^{2}$ & $-2 \omega^{2}$ & abcde \\
\hline 45 & 2 & $i(3-\sqrt{ } 5) \omega^{2}$ & $i(-3+\sqrt{ } 5) \omega^{2}$ & $-2 \omega$ & $a d e b c$ \\
\hline 46 & $\sqrt{ } 3-i \sqrt{ } 5$ & $2 \omega^{2}$ & $-2 \omega^{2}$ & $(\sqrt{ } 3+i \sqrt{ } 5) \omega$ & $a e c d b$ \\
\hline 47 & $-\sqrt{ } 3-i \sqrt{ } 5$ & $2 \omega$ & $-2 \omega$ & $(-\sqrt{ } 3+i \sqrt{ } 5) \omega^{2}$ & abdce \\
\hline 48 & 2 & $i(3-\sqrt{ } \tilde{5}) \omega$ & $i(-3+\sqrt{ } 5) \omega$ & $-2 \omega^{2}$ & acbed \\
\hline 49 & 2 & $i(-3-\sqrt{ } 5) \omega$ & $i(3+\sqrt{ } 5) \omega$ & $-2 \omega^{2}$ & $a c d e b$ \\
\hline 50 & $\sqrt{ }^{3}-i \sqrt{ } 5$ & $2 \omega$ & $-2 \omega$ & $(\sqrt{ } 3+i \sqrt{ } 5) \omega^{2}$ & adbce \\
\hline 51 & $-\sqrt{ } 3-i \sqrt{ } 5$ & $2 \omega^{2}$ & $-2 \omega^{2}$ & $(-\sqrt{3}+i \sqrt{5}) \omega$ & $a e c b d$ \\
\hline 52 & 2 & $i(-3-\sqrt{ } 5) \omega^{2}$ & $i(3+\sqrt{ } 5) \omega^{2}$ & $-2 \omega$ & abede \\
\hline 53 & 2 & $i(-3-\sqrt{5}) \omega$ & $i(3+\sqrt{ } 5)$ & $-2 \omega$ & aebcd \\
\hline 54 & $-\sqrt{ } 3-i \sqrt{ } 5$ & $2 \omega$ & -2 & $(-\sqrt{ } 3+i \sqrt{ } 5) \omega$ & abdec \\
\hline 55 & $\sqrt{ } 3-i \sqrt{ } 5$ & 2 & $-2 \omega^{2}$ & $(\sqrt{ } 3+i \sqrt{ } 5) \omega^{2}$ & $a c e d b$ \\
\hline 56 & 2 & $i(-3-\sqrt{ } 5)$ & $i(3+\sqrt{ } 5) \omega^{2}$ & $-2 \omega^{2}$ & adcbe \\
\hline 57 & 2 & $i(-3-\sqrt{ } 5)$ & $i(3+\sqrt{ } / 5) \omega$ & $-2 \omega$ & $a d e c b$ \\
\hline 58 & $-\sqrt{ } 3-i \sqrt{ } 5$ & 2 & $-2 \omega$ & $(-\sqrt{ } 3+i \sqrt{ } 5) \omega$ & $a e b d c$ \\
\hline 59 & $\sqrt{ } 3-i \sqrt{ } 5$ & $2 \omega^{2}$ & -2 & $(\sqrt{ } 3+i \sqrt{ } 5) \omega^{2}$ & acdbe \\
\hline 60 & 2 & $i(-3-\sqrt{ } 5) \omega^{2}$ & $i(3+\sqrt{ } 5)$ & $-2 \omega^{2}$ & abced \\
\hline
\end{tabular}


115. We hence derive

Homographic Transformations. The groups of 12 and 24. Pole at $B$.
$(A s$
$+B)$
$\div(\mathrm{Cs}$
$+D)$

\begin{tabular}{|c|c|c|c|c|c|}
\hline 1 & 1 & 0 & 0 & 1 & 1 \\
\hline 2 & 2 & $i(3-\sqrt{ } 5)$ & $i(-3+\sqrt{ } 5)$ & -2 & $a b . c d$ \\
\hline 3 & 0 & & & 0 & $a c \cdot b d$ \\
\hline 4 & 2 & $i(-3-\sqrt{ } 5)$ & $i(3+\sqrt{ } 5)$ & -2 & $a d . b c$ \\
\hline 5 & $2 \omega^{2}$ & $-\sqrt{ } 3-i \sqrt{ } 5$ & $-\sqrt{ } 3+i \sqrt{ } 5$ & $-2 \omega$ & $a b c$ \\
\hline 6 & $2 \omega$ & $-\sqrt{ } 3-i \sqrt{ } 5$ & $\sqrt{3}+i \sqrt{ } 5$ & $-2 \omega^{2}$ & $a c b$ \\
\hline 7 & $-\sqrt{ } 3-i \sqrt{ } 5$ & & $-2 \omega^{2}$ & $-\sqrt{ } 3+i \sqrt{ } 5$ & $a b d$ \\
\hline 8 & $\sqrt{3}-i \sqrt{ } 5$ & & $-2 \omega^{2}$ & $\sqrt{3}+i \sqrt{ } 5$ & $a d b$ \\
\hline 9 & & $\sqrt{ } 3-i \sqrt{ } 5$ & $\sqrt{ } 3+i \sqrt{ } 5$ & $-2 \omega^{2}$ & acd \\
\hline 10 & & $\sqrt{ } 3-i \sqrt{ } 5$ & $\sqrt{ } 3+i \sqrt{ } 5$ & $-2 \omega$ & $a d c$ \\
\hline 11 & $\sqrt{ } 3-i \sqrt{ } 5$ & $2 \omega^{2}$ & $-2 \omega$ & $\sqrt{ } 3+i \sqrt{ } 5$ & $b c d$ \\
\hline 12 & $-\sqrt{ } 3-i \sqrt{ } 5$ & $2 \omega^{2}$ & $-2 \omega$ & $-\sqrt{ } 3+i \sqrt{ } 5$ & $b d c$ \\
\hline 13 & 2 & $\sqrt{ } 3(1+\sqrt{ } 5)+(-3-\sqrt{ } 5)$ & $\sqrt{ } 3(1+\sqrt{ } 5)+i(3+\sqrt{ } 5)$ & -2 & $a b$ \\
\hline 14 & 2 & $\sqrt{ } 3(-1-\sqrt{ } 5)+(-3-\sqrt{ } 5)$ & $\sqrt{ } 3(-1-\sqrt{ } 5)+i(3+\sqrt{ } 5)$ & -2 & $c d$ \\
\hline 15 & $\sqrt{ } 5$ & $-i$ & & $-\sqrt{ } 5$ & $a c$ \\
\hline 16 & 1 & $i \sqrt{ } 5$ & $-i \sqrt{ } 5$ & -1 & $b d$ \\
\hline 17 & 2 & $\sqrt{ } 3(-1+\sqrt{ } 5)+i(3-\sqrt{5})$ & $\sqrt{ } 3(-1+\sqrt{ } 5)+i(-3+\sqrt{ } 5)$ & -2 & $a d$ \\
\hline 18 & 2 & $\sqrt{ } 3(1-\sqrt{ } 5)+i(3-\sqrt{ } 5)$ & $\sqrt{ } 3(1-\sqrt{ } 5)+i(-3+\sqrt{ } 5)$ & -2 & $b c$ \\
\hline 19 & 1 & $i$ & & 1 & abcd \\
\hline 20 & 1 & $-i$ & $-i$ & 1 & $a d c b$ \\
\hline 21 & $\sqrt{ } 3(1-\sqrt{ } 5)+i(3+\sqrt{ } 5)$ & 2 & -2 & $\sqrt{ } 3(1-\sqrt{ } 5)+i(-3+\sqrt{ } 5)$ & $a b d c$ \\
\hline 22 & $\sqrt{ } 3(1+\sqrt{ } 5)+i(-3+\sqrt{ } 5)$ & 2 & -2 & $\sqrt{ } 3(1+\sqrt{ } 5) \div i(3+\sqrt{ } 5)$ & $a c b d$ \\
\hline 23 & $\sqrt{ } 3(-1+\sqrt{ } 5)+i(3-\sqrt{ } 5)$ & 2 & -2 & $\sqrt{ } 3(-1+\sqrt{ } 5)+i(-3+\sqrt{5})$ & $a c d b$ \\
\hline 24 & $\sqrt{ } 3(-1-\sqrt{ } 5)+i(-3-\sqrt{ } 5)$ & 2 & -2 & $\sqrt{ } 3(-1-\sqrt{ } 5)+i(3+\sqrt{ } 5)$ & $a d b c$ \\
\hline
\end{tabular}

116. I give also the group of $12,(a b c e)$, slightly modifying the form: viz. I write first $\sqrt{ } 3+i \sqrt{ } 5=2 \sqrt{ } 2 k$, and therefore $\sqrt{ } 3-i \sqrt{ } 5=2 \sqrt{ } 2 \cdot \frac{1}{k}$ : then for $x$ I write $\lambda x$, and divide the $A$ and $B$ by $\lambda$ : the $A$ and $B$ then contain $\frac{k}{\lambda}$, and the $C$ and $D$ contain $\frac{\lambda}{k}$, and assuming $\frac{k}{\lambda}=i$, we have $\frac{\lambda}{k}=-i$. For instance, in the transformation corresponding to $a b c$, the $A x+B$ and $C x+D$,

$$
=2 \omega^{2} x-(\sqrt{ } 3+i \sqrt{ } 5) \text { and }(-\sqrt{ } 3+2 \sqrt{ } 5) x-2 \omega,
$$

become first $2 \omega^{2} x-2 \sqrt{ } 2 k$, and $-2 \sqrt{ } 2 \frac{1}{k} x-2 \omega$, and then (omitting also the factor 2) $\omega^{2} x-\sqrt{ } 2 \frac{k}{\lambda}$ and $-\sqrt{ } 2 \frac{\lambda}{k} x-\omega$, viz. when $\frac{k}{\lambda}=i$, they are $\omega^{2} x-i \sqrt{ } 2$ and $x . i \sqrt{ } 2-\omega ;$ that is, the values of $A, B, C, D$ are $\omega^{2},-i \sqrt{ } 2, i \sqrt{ } 2,-\omega$. The group is 
Group of 12 . Pole at $B$.

\begin{tabular}{|l|l|l|l|l|}
\hline 1 & 0 & 0 & 1 & 1 \\
$\omega$ & 0 & 0 & 1 & $a c e$ \\
$\omega^{2}$ & 0 & 0 & 1 & $a c c$ \\
1 & $-i \omega \sqrt{ } 2$ & $i \omega \sqrt{ } 2$ & $-\omega^{2}$ & $a b c$ \\
1 & $-i \omega^{2} \sqrt{ } 2$ & $i \omega^{2} \sqrt{ } 2$ & $-\omega$ & $a c b$ \\
1 & $-i-\omega \sqrt{ } 2$ & $i \sqrt{ } 2$ & $-\omega$ & $a b e$ \\
1 & $-i \sqrt{ } 2$ & $i \omega^{2} \sqrt{ } 2$ & $-\omega^{2}$ & $a e b$ \\
1 & $-i \omega^{2} \sqrt{ } 2$ & $i \sqrt{ } 2$ & $-\omega^{2}$ & $b e c$ \\
1 & $-i \sqrt{ } 2$ & $i \omega \sqrt{ } 2$ & $-\omega$ & $b c e$ \\
1 & $-i \omega \sqrt{ } 2$ & $i \omega^{2} \sqrt{ } 2$ & -1 & $a b \cdot c e$ \\
1 & $-i \omega^{2} \sqrt{ } 2$ & $i \omega \sqrt{ } 2$ & -1 & $a e \cdot b c$ \\
1 & $-i \sqrt{ } 2$ & $i \sqrt{ } 2$ & -1 & $a c \cdot b e$ \\
\hline
\end{tabular}

117. From the Table of the Groups of 12 and 24, $\Theta$-form, it appears that the group of 12 is

$$
\begin{gathered}
x, \frac{1}{x},-x,-\frac{1}{x}, \frac{i(x-1)}{x+1}, \frac{-i(x-1)}{x+1}, \frac{i(x+1)}{x-1}, \frac{-i(x+1)}{x-1}, \\
\frac{x+i}{x-i}, \frac{x-i}{x+i}, \frac{-(x+i)}{x-i}, \frac{-(x-i)}{x+i}
\end{gathered}
$$

and if we proceed to form the product of the twelve factors $s-x, s-\frac{1}{x}, s+x$, \&c., we have first the three products

$$
\begin{array}{lrc}
s^{2}-x^{2} \cdot s^{2}-\frac{1}{x^{2}} ; & s^{2}+\left(\frac{x-1}{x+1}\right)^{2} \cdot s^{2}+\left(\frac{x+1}{x-1}\right)^{2} ; & s^{2}-\left(\frac{x+i}{x-i}\right)^{2} \cdot s^{2}-\left(\frac{x-i}{x+i}\right)^{2} \\
=s^{4}+\alpha s^{2}+1 ; & s^{4}+\beta s^{2}+1 ; & s^{4}+\gamma s^{2}+1 ;
\end{array}
$$

if for shortness

$$
\alpha, \beta, \gamma=-\left(x^{2}+\frac{1}{x^{2}}\right), \quad 2 \frac{x^{4}+6 x^{2}+1}{\left(x^{2}-1\right)^{2}},-2 \frac{x^{4}-6 x^{2}+1}{\left(x^{2}+1\right)^{2}} .
$$

The product of the three quartic functions is

$$
=\left(s^{4}+1\right)^{3}+\left(s^{4}+1\right)^{2} s^{2}(\alpha+\beta+\gamma)+\left(s^{4}+1\right) s^{4}(\beta \gamma+\gamma \alpha+\alpha \beta)+s^{6} \cdot \alpha^{2} \beta^{2} \gamma^{2} ;
$$

and we have

$$
\begin{gathered}
\beta+\gamma=\frac{32 x^{2}\left(x^{4}+1\right)}{\left(x^{4}-1\right)^{2}}, \quad \alpha+\beta+\gamma=\frac{-\left(x^{12}-33 s^{8}-33 s^{4}+1\right)}{x^{2}\left(x^{4}-1\right)^{2}}, \\
\beta \gamma=\frac{-4\left(x^{8}-34 x^{4}+1\right)}{\left(x^{4}-1\right)^{2}}, \quad \alpha(\beta+\gamma)=\frac{-32 x^{2}\left(x^{4}+1\right)^{2}}{x^{2}\left(x^{4}-1\right)^{2}}, \\
\beta \gamma+\gamma \alpha+\alpha \beta,=\frac{-36 x^{2}\left(x^{4}-1\right)^{2}}{x^{2}\left(x^{4}-1\right)^{2}},=-36, \quad \alpha \beta \gamma=\frac{4\left(x^{12}-33 s^{8}-33 s^{4}+1\right)}{x^{2}\left(x^{4}-1\right)^{2}} .
\end{gathered}
$$

Hence the product is found to be

$$
=\left(s^{12}-33 s^{8}-33 s^{4}+1\right)-s^{2}\left(s^{4}-1\right)^{2} \cdot \frac{x^{12}-33 x^{8}-33 x^{4}+1}{x^{2}\left(x^{4}-1\right)^{2}},
$$


which is

$$
=s^{2}\left(s^{4}-1\right)^{2}\left\{\frac{s^{12}-33 s^{8}-33 s^{4}+1}{s^{2}\left(s^{4}-1\right)^{2}}-\frac{x^{12}-33 x^{8}-33 x^{4}+1}{x^{2}\left(x^{4}-1\right)^{2}}\right\} .
$$

We thus verify that the twelve transformations $x$ into $x$, into $\frac{1}{x}$, \&c., give each of them a transformation of the function

into itself.

$$
\frac{x^{12}-33 x^{8}-33 x^{4}+1}{x^{2}\left(x^{4}-1\right)^{2}}
$$

The system of 15 circles. Art. Nos. 118 to 127 .

118. It has been already remarked that we can from the coefficients $(A, B, C, D)$ of the homographic transformation pass back to the position of the axis of rotation: viz. we have

and thence

$$
A: B: C: D=-\nu-i: \lambda+i \mu: \lambda-i \mu: \nu-i,
$$

that is,

$$
\lambda: \mu: \nu: 1=\quad B+C:-i(B-C) \quad: D-A: i(D+A),
$$

$$
\lambda, \mu, \nu=-i(B+C), \quad-(B-C),-i(D-A) ; \quad \div(D+A) .
$$

The equations of the axis thus are

$$
\frac{x}{B+C}=\frac{i y}{B-C}=\frac{z}{D-A},
$$

and the equations of the central plane at right angles to the axis are

$$
-(B+C) x+i(B-C) y+(A-D) z=0 .
$$

119. In particular, we may find the equations of the 15 planes at right angles to the $\Theta$-axes: these are in fact the before-mentioned 15 planes, intersecting the sphere in great circles the projections of which are the circles in the three figures respectively. Taking the equation of the plane to be $L x+M y+N z=0$, it is at once seen that the equation of the projecting cone (vertex at the South pole) is

$$
N\left(x^{2}+y^{2}+z^{2}-1\right)-2(z+1)(L x+M y+N z)=0,
$$

and hence, writing $z=0$, we find

$$
N\left(x^{2}+y^{2}-1\right)-2(L x+M y)=0
$$

for the equation of the circle in the plane figure. We have thus the equations of a system of 15 circles related to each other in the manner before referred to.

120. Taking the $\Theta$-form, the equations of the 15 planes are at once found: and we thence obtain the equations of the 15 circles: viz. writing for shortness

$$
\Omega=x^{2}+y^{2}-1 \text {, }
$$


the equations are

$$
\begin{array}{lll}
z=0, & (a b . c d) & \Omega=0, \\
x=0, & (a c . b d) & x=0, \\
y=0, & (a d . b c) & y=0,
\end{array}
$$

$$
\begin{aligned}
& (3-\sqrt{ } 5) x+(1-\sqrt{ } 5) y+2 z=0, \quad(a e . b c) \\
& (-1-\sqrt{ } 5) x+(-1+\sqrt{ } 5) y+2 z=0, \quad(a b . c e) \\
& (1+\sqrt{ } 5) x+(3+\sqrt{ } 5) y+2 z=0, \quad(a c . b e) \\
& (-3+\sqrt{ } 5) x+(-1+\sqrt{ } 5) y+2 z=0, \quad(a d . b e) \\
& (1+\sqrt{ } 5) x+(1-\sqrt{ } 5) y+2 z=0, \quad(a b . d e) \\
& (-1-\sqrt{ } 5) x+(-3-\sqrt{ } 5) y+2 z=0, \quad(a e . b d) \\
& (-3+\sqrt{ } 5) x+(1-\sqrt{ } 5) y+2 z=0, \quad(a d . c e) \\
& (1+\sqrt{ } 5) x+(-1+\sqrt{ } 5) y+2 z=0, \quad(a e . c d) \\
& (-1-\sqrt{ } 5) x+(3+\sqrt{ } 5) y+2 z=0, \quad(a c . d e) \\
& (3-\sqrt{ } 5) x+(-1+\sqrt{ } 5) y+2 z=0, \quad(b c . d e) \\
& (-1-\sqrt{ } 5) x+(1-\sqrt{ } 5) y+2 z=0, \quad(b e . c d) \\
& (1+\sqrt{ } 5) x+(-3-\sqrt{ } 5) y+2 z=0, \quad(b d . c e) .
\end{aligned}
$$$$
\Omega-[(3-\sqrt{ } 5) x+(1-\sqrt{ } 5) y]=0,
$$$$
\Omega-[(-1-\sqrt{ } 5) x+(-1+\sqrt{ } 5) y]=0,
$$$$
\Omega-[(1+\sqrt{ } 5) x+(3+\sqrt{ } 5) y]=0 ;
$$$$
\text { and similarly for the other circles. }
$$

121. Observe that the arrangement is in sets of 3 planes, or circles, intersecting at right angles. One of the circles is the circle $\Omega,=x^{2}+y^{2}-1,=0$ corresponding to the equator, and two of them are the right lines $x=0$ and $y=0$. The equations of the remaining 12 circles may be written in the somewhat different form

$$
\begin{aligned}
& \Omega+(\sqrt{ } 5-1)\left[y-\frac{1}{2}(\sqrt{ } 5-1) x\right]=0, \\
& \Omega-(\sqrt{ } 5-1)\left[y-\frac{1}{2}(\sqrt{ } 5+3) x\right]=0, \\
& \Omega-(\sqrt{ } 5+3)\left[y+\frac{1}{2}(\sqrt{ } 5-1) x\right]=0, \\
& \Omega-(\sqrt{ } 5-1)\left[y-\frac{1}{2}(\sqrt{ } 5-1) x\right]=0, \\
& \Omega+(\sqrt{ } 5-1)\left[y-\frac{1}{2}(\sqrt{ } 5+3) x\right]=0, \\
& \Omega+(\sqrt{ } 5+3)\left[y+\frac{1}{2}(\sqrt{ } 5-1) x\right]=0, \\
& \Omega+(\sqrt{ } 5-1)\left[y+\frac{1}{2}(\sqrt{ } 5-1) x\right]=0, \\
& \Omega-(\sqrt{ } 5-1)\left[y+\frac{1}{2}(\sqrt{ } 5+3) x\right]=0, \\
& \Omega-(\sqrt{ } 5+3)\left[y-\frac{1}{2}(\sqrt{ } 5-1) x\right]=0, \\
& \Omega-(\sqrt{ } 5-1)\left[y+\frac{1}{2}(\sqrt{ } 5-1) x\right]=0, \\
& \Omega+(\sqrt{ } 5-1)\left[y+\frac{1}{2}(\sqrt{ } 5+3) x\right]=0, \\
& \Omega+(\sqrt{ } 5+3)\left[y-\frac{1}{2}(\sqrt{ } 5-1) x\right]=0 .
\end{aligned}
$$

It hence appears that 4 and 4 circles have with $\Omega=0$ the common chords $y+\frac{1}{2}(\sqrt{ } 5-1) x=0$, $y-\frac{1}{2}(\sqrt{ } 5-1) x=0$ respectively: and that 2 and 2 circles have with $\Omega=0$ the common chords $y+\frac{1}{2}(\sqrt{ } 5+3) x=0, y-\frac{1}{2}(\sqrt{ } 5+3) x=0$ respectively.

C. XI. 
122. The equations of the 12 circles are, in fact,

$$
\begin{aligned}
& \Omega \pm(\sqrt{ } 5-1)\left[y \pm \frac{1}{2}(\sqrt{ } 5-1) x\right]=0, \quad \Omega \pm(\sqrt{ } 5+3)\left[y \pm \frac{1}{2}(\sqrt{ } 5-1) x\right]=0, \\
& \Omega \pm(\sqrt{ } 5-1)\left[y \pm \frac{1}{2}(\sqrt{ } 5+3) x\right]=0:
\end{aligned}
$$

hence the radii are $=\sqrt{ } 5-1,2$ and $\sqrt{ } 5+1$ respectively.

The construction of the 12 circles is as follows. Starting with a circle radius 1 .

Lay down the diameters $y \pm \frac{1}{2}(\sqrt{ } 5-1) x=0$ ( $A A$ in the figure), and through the extremities of each describe 2 pairs of circles with the radii $\sqrt{ } 5-1, \sqrt{ } 5+1$ respectively.

Lay down the diameters $y \pm \frac{1}{2}(\sqrt{ } 5+3) x=0 \quad(B B$ in the figure), and through the extremities of each describe a pair of circles with the radius 2 .

123. For the $A$-form, the equations of the fifteen planes are at once found to be

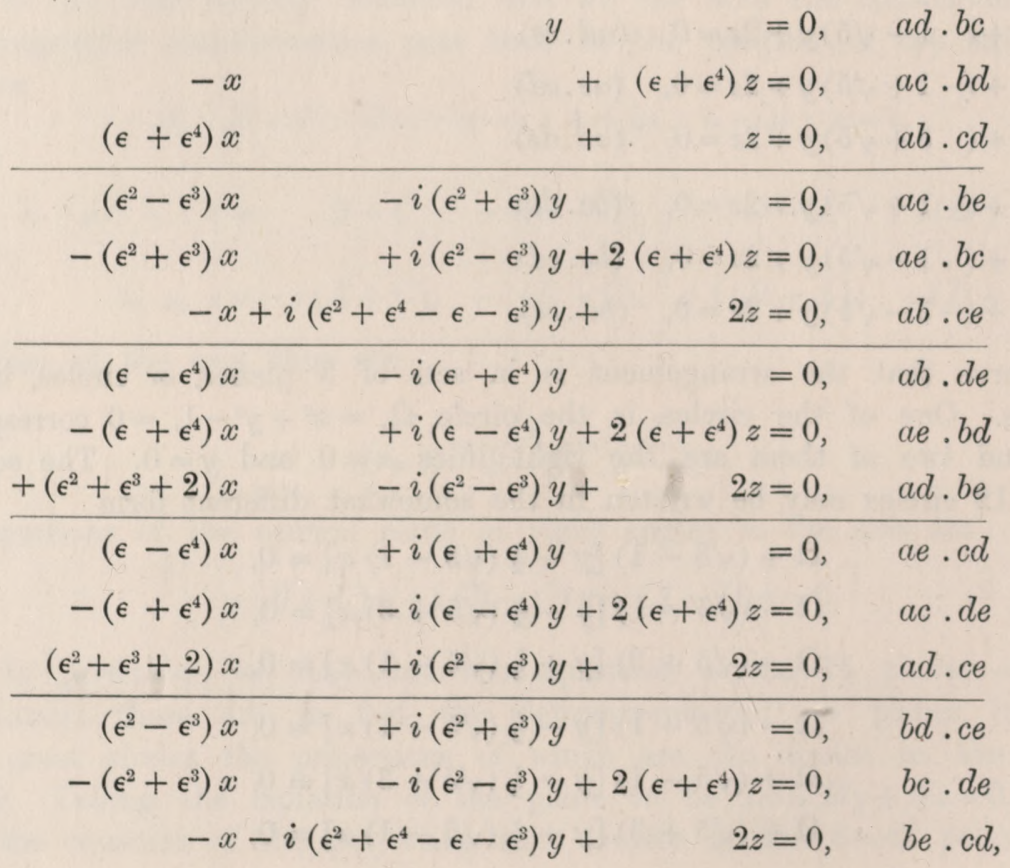

where, as before, the three planes of each set intersect at right angles.

124. Passing to the circles, the first plane of each set gives a right line, and we have thus five of the circles reducing themselves to right lines inclined to the axis of $x$ at angles $0^{\circ}, 36^{\circ}, 72^{\circ}, 108^{\circ}$ and $144^{\circ}$ respectively.

The remaining 10 circles form 5 pairs, the circles of a pair having different radii, but the two radii being the same for each pair, and so that for the several pairs the common chords with the circle $\Omega=0$, are the diameters inclined to the axis of $x$ at the angles $18^{\circ}, 54^{\circ}, 90^{\circ}, 126^{\circ}$ and $162^{\circ}$ respectively. Considering the two circles for which the inclination is $90^{\circ}$, these arise from the planes $-x+\left(\epsilon+\epsilon^{4}\right) z=0$, $\left(\epsilon+\epsilon^{4}\right) x+z=0$ respectively. The equations of the circles thus are $\left(\epsilon+\epsilon^{4}\right) \Omega+2 x=0$, 
$\Omega-2\left(\epsilon+\epsilon^{4}\right) x=0$, or recollecting that $2\left(\epsilon+\epsilon^{4}\right)=\sqrt{ } 5-1$ and therefore $\frac{2}{\epsilon+\epsilon^{4}}=\sqrt{ } 5+1$, the equations are

$$
x^{2}+y^{2}-(\sqrt{ } 5-1) x-1=0, \quad x^{2}+y^{2}+(\sqrt{ } 5+1) x=0 ;
$$

hence for the first circle the $x$-coordinate of the centre is $\frac{1}{2}(\sqrt{ } 5-1)$ and the radius is $=\frac{1}{2} \sqrt{ }(10-2 \sqrt{ } 5)$; for the second circle the $x$-coordinate of the centre is $=\frac{1}{2}(\sqrt{ } 5+1)$, and the radius $=\frac{1}{2} \sqrt{ }(10+2 \sqrt{5})$. We have thus the construction of these two circles, and consequently the construction of all the 12 circles.

125. For the $B$-form, after some easy reductions and attending to the relation $\omega-\omega^{2}=i \sqrt{ } 3$, the equations of the 15 planes become

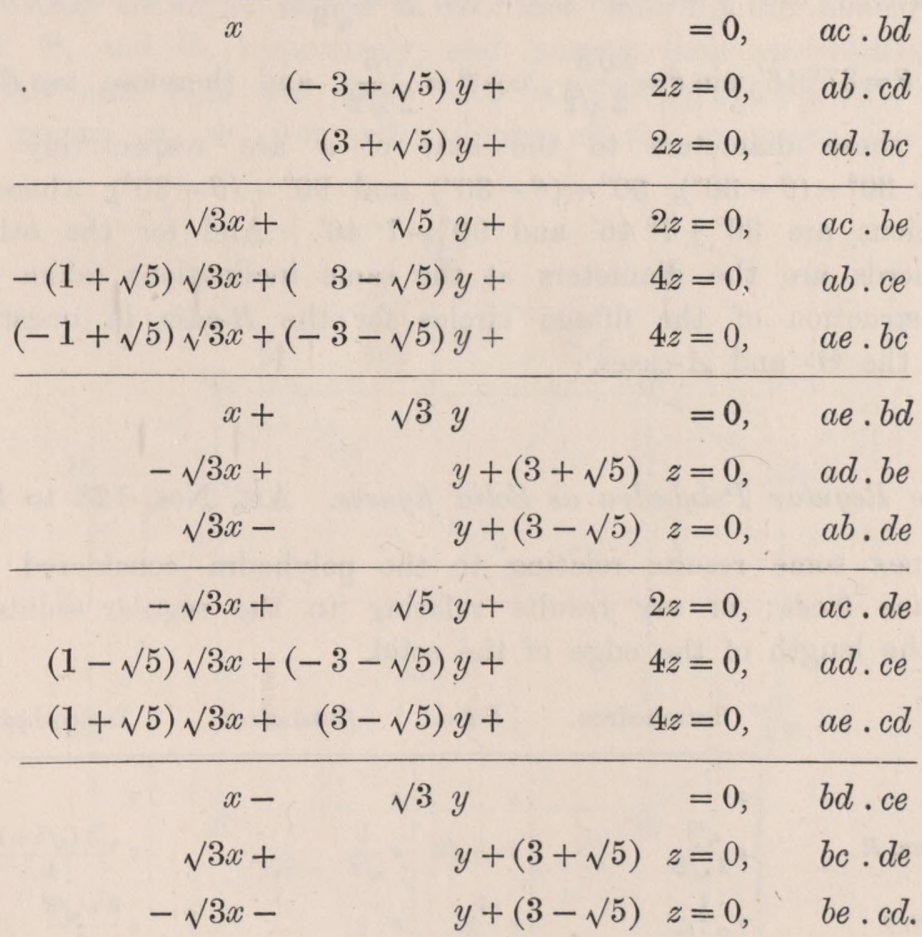

126. Of the 15 circles, 3 are the lines $x-y \sqrt{ } 3=0, x=0, x+y \sqrt{ } 3=0$, viz. these are lines at inclinations $30^{\circ}, 90^{\circ}, 150^{\circ}$ to the axis of $x$. The equations of the remaining 12 circles are

$$
\begin{array}{r}
\Omega+(3-\sqrt{ } 5) y=0, \\
\Omega-(3+\sqrt{ } 5) y=0, \\
(3+\sqrt{ } 5) \Omega-2(y-x \sqrt{ } 3)=0 \\
(3-\sqrt{ } 5) \Omega+2(y-x \sqrt{ } 3)=0 \\
(3+\sqrt{ } 5) \Omega-2(y+x \sqrt{ } 3)=0 \\
(3-\sqrt{ } 5) \Omega+2(y+x \sqrt{ } 3)=0,
\end{array}
$$


viz. these are pairs of circles having, for their common chords with $\Omega=0$, the diameters at inclinations $0^{\circ}, 60^{\circ}, 120^{\circ}$ respectively. And, lastly, we have the circles

$$
\begin{array}{rl|rrr}
2 \Omega-[(-1+\sqrt{ } 5) \sqrt{ } 3 x-(3+\sqrt{ } 5) y] & =0, & 2 \Omega+[(-1+\sqrt{ } 5) \sqrt{ } 3 x+(3+\sqrt{ } 5) y] & =0, \\
\Omega-[-\sqrt{ } 3 x+\sqrt{ } 5 y] & =0, & \Omega-[ & \sqrt{ } 3 x+\sqrt{ } 5 y]=0, \\
2 \Omega+[(1+\sqrt{ } 5) \sqrt{ } 3 x-(3-\sqrt{ } 5) y] & =0, & 2 \Omega-[( & 1+\sqrt{ } 5) \sqrt{ } 3 x+(3-\sqrt{ } 5) y] & =0 .
\end{array}
$$

127. The first three of these have, for common chords with $\Omega=0$, the diameters whose equations are

$$
(-1+\sqrt{ } 5) \sqrt{ } 3 x-(3+\sqrt{ } 5) y=0, \quad-\sqrt{ } 3 x+\sqrt{ } 5 y=0, \quad(1+\sqrt{ } 5) \sqrt{ } 3 x-(3-\sqrt{ } 5) y=0:
$$

viz. these equations are $y=(-2+\sqrt{ } 5) x \sqrt{ } 3, y=\frac{\sqrt{ } 3}{\sqrt{ } 5} x, y=(2+\sqrt{ } 5) x \sqrt{ } 3$. If, as in a foregoing table, $\theta=37^{\circ} 46^{\prime}, \sin \theta=\frac{\sqrt{ } 3}{2 \sqrt{ } 2}, \cos \theta=\frac{\sqrt{ } 5}{2 \sqrt{ } 2}$, and therefore $\tan \theta=\frac{\sqrt{ } 3}{\sqrt{ } 5}$; then the inclinations of these diameters to the axis of $x$ are respectively $60^{\circ}-\theta, \theta$ and $120^{\circ}-\theta$, or say $30^{\circ}-\left(\theta-30^{\circ}\right), 30^{\circ}+\left(\theta-30^{\circ}\right)$ and $90^{\circ}-\left(\theta-30^{\circ}\right)$, where $\theta-30^{\circ}=7^{\circ} 46^{\prime}$, i.e. the inclinations are $30^{\circ} \pm 7^{\circ} 46^{\prime}$ and $90^{\circ}-7^{\circ} 46^{\prime}$. And for the other three circles the common chords are the diameters at the same inclinations taken negatively. The geometrical construction of the fifteen circles for the $B$-case in question is thus not so simple as in the $\Theta$ - and $A$-cases.

The Regular Polyhedra as Solid figures. Art. Nos. 128 to 134.

128. I annex some results relating to the polyhedra considered as solid figures bounded by plane faces; or say results relating to the regular solids: $s$ is in each case taken for the length of the edge of the solid.

Tetrahedron. Cube. Octahedron. Dodecahedron. Icosahedron.

\section{Edge}

Rad. of circum. sphere, $R$

\begin{tabular}{|l|l|l|l|l|}
\hline$s$ & $s$ & $s$ & $s$ & $s$ \\
$s \frac{\sqrt{ } 3}{2 \sqrt{ } 2}$ & $s \cdot \frac{1}{2} \sqrt{ } 3$ & $s \frac{1}{\sqrt{ } 2}$ & $s \frac{\sqrt{ } 3(\sqrt{ } 5+1)}{4}$ & $s \sqrt{\frac{5+\sqrt{ } 5}{8}}$ \\
$s \frac{1}{2 \sqrt{ } 2}$ & $s \frac{1}{\sqrt{ } 2}$ & $s \cdot \frac{1}{2}$ & $s \frac{1+\sqrt{ } 5}{4}$ \\
$s \frac{1}{2 \sqrt{ } 2 \sqrt{ } 3}$ & $s \cdot \frac{1}{2}$ & $s \frac{1}{\sqrt{ } 2 \sqrt{ } 3}$ & $s \sqrt{\frac{25+11 \sqrt{ } 5}{40}}$ & $s \frac{3+\sqrt{ } 5}{4 \sqrt{ } 3}$ \\
$s \cdot \frac{1}{\sqrt{ } 3}$ & $s \frac{1}{\sqrt{ } 2}$ & $s \frac{1}{\sqrt{ } 3}$ & $s \frac{1}{\frac{5+\sqrt{ } 5}{10}}$ & $s \frac{1}{\sqrt{3}}$ \\
$s \cdot \frac{1}{2 \sqrt{ } 3}$ & $s \cdot \frac{1}{2}$ & $s \frac{1}{2 \sqrt{ } 3}$ & $s \frac{1}{2 \sqrt{ } 3}$ \\
$\cos ^{-1} \frac{1}{3}=70^{\circ} 28^{\prime}$ & $90^{\circ}$ & $\cos ^{-1}-\frac{1}{3}=109^{\circ} 32^{\prime}$ & & \\
$\cos ^{-1} \frac{1}{\sqrt{ } 3}=54^{\circ} 46^{\prime}$ & $90^{\circ}$ & $\cos ^{-1}-\frac{1}{\sqrt{ } 3}=125^{\circ} 44^{\prime}$ & & \\
\hline
\end{tabular}

Rad. of inters. sphere, $\rho$

Rad. of inscribed sphere, $r$

Rad. of circle circum. to face, $R^{\prime}$

Rad. of circle inscribed to face, $r^{\prime}$

Incl. of adjacent faces

Incl. of edge to adjacent face

But we require further data in the cases of the dodecahedron and the icosahedron respectively. 
129. For the dodecahedron, taking the edge to be $=s$ as before, then in the pentagonal face

where

$$
\begin{aligned}
\text { diagonal, } g \text { is } & =s \cdot \frac{1}{2}(\sqrt{ } 5+1), \\
\text { altitude, } k ” & =s \cdot \frac{1}{2} \sqrt{ }(5+2 \sqrt{ } 5), \\
\text { segments of do., } e ” & =s \cdot \frac{1}{4} \sqrt{ }(10-2 \sqrt{ } 5), \\
f ” & =s \cdot \frac{1}{4} \sqrt{ }(10+2 \sqrt{ } 5),
\end{aligned}
$$

$$
k=e+f=R^{\prime}+r^{\prime} .
$$

130. The section through a pair of opposite edges is a hexagon, as shown in the figure, viz. this is constructed by taking the four equal distances $0 \Theta,=\rho$, $=s \cdot \frac{1}{4}(3+\sqrt{ } 5)$, meeting at right angles in $O$; then drawing the double ordinates $B B$, each $=s$, through $\Theta_{1}$ and $\Theta_{3}$ respectively, and joining their extremities with $\Theta_{2}$ and $\Theta_{4}$ : the sides $\Theta_{2} B$ and $\Theta_{4} B$ are then each $=k,=s \cdot \frac{1}{2} \sqrt{ }(5+2 \sqrt{ } 5)$; and inserting upon them the points $A, \Phi$ from the figure of the pentagon, we have several

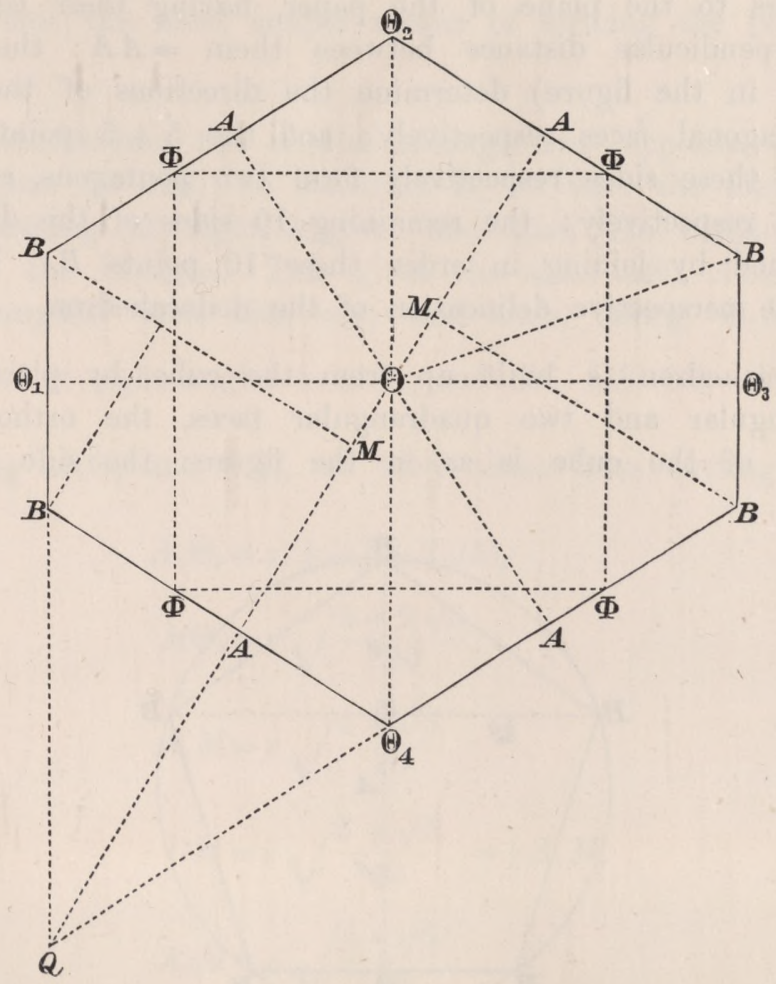

geometrical relations; viz. the line $A A$ cuts the parallel sides $B \Theta_{2}, B \Theta_{4}$ at right angles, and when produced passes through the intersection of $B \Theta_{1}$ and $B \Theta_{4}$ : we have $O A, O B, O \Theta=r, R, \rho$ respectively: the four points $\Phi$ form a square, the side of which is $g,=s \cdot \frac{1}{2}(\sqrt{ } 5+1)$. 
131. We find also

$$
\begin{aligned}
& A M=s \sqrt{\frac{5+\sqrt{ } 5}{10}} \\
& A Q=s \sqrt{\frac{5+2 \sqrt{ } 5}{5}} \\
& Q M=s \sqrt{\frac{25+11 \sqrt{ } 5}{5}},=r \cdot 2 \sqrt{ } 2, \\
& O Q=s \sqrt{\frac{25+11 \sqrt{ } 5}{8}},=r \cdot \sqrt{ } 5 \\
& O M=s \sqrt{\frac{5-\sqrt{ } 5}{40}} \\
& M B=s \sqrt{\frac{2(5+2 \sqrt{ } 5)}{B} .}
\end{aligned}
$$

It may be remarked that in the figure $B \Theta_{2}, B \Theta_{4}$ are the projections of pentagonal faces, at right angles to the plane of the paper, having their centres at the points $A, A$, and the perpendicular distance between them $=A A$ : the points $Q, Q$ (only one of them shown in the figure) determine the directions of the $5+5$ sides which abut on these pentagonal faces respectively; and the $5+5$ points $B$ which are the other extremities of these sides respectively form two pentagons, centres $M, M$ in the planes $M B$ and $M B$ respectively: the remaining 10 sides of the dodecahedron are the skew decagon obtained by joining in order these 10 points $B$. We have thus the means of making the perspective delineation of the dodecahedron.

132. The dodecahedron is built up from the cube, by placing on each face a figure of two triangular and two quadrangular faces, the orthogonal projection of which on the face of the cube is as in the figure: the side of the square is $g$,

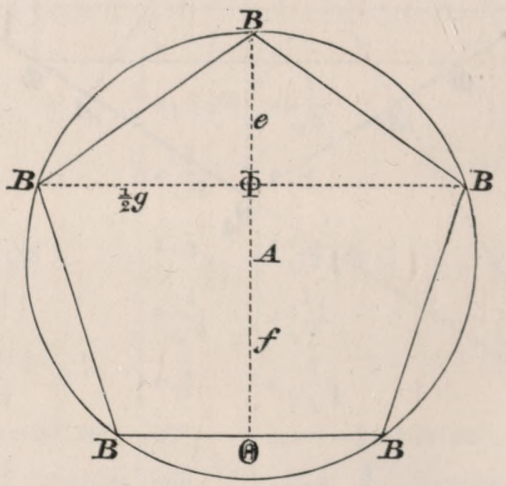

$=s \cdot \frac{1}{2}(\sqrt{ } 5+1):$ the slope-breadths of the triangular faces are $e,=s \cdot \frac{1}{4} \sqrt{ }(10-2 \sqrt{ } 5)$, and those of the quadrangular faces are $f,=s \cdot \frac{1}{4} \sqrt{ }(10+2 \sqrt{5})$; the lines represented by the other lines of the figure are in actual length each $=s$. We have thus a 
section which is an isosceles triangle, base $=g$, other sides each $=f$; and the square of the altitude is thus $=f^{2}-\frac{1}{4} g^{2}=\frac{1}{4} s^{2}$, or the altitude $=\frac{1}{2} s$; viz. the altitude of the ridge-line $B B$, above the face of the cube is $=\frac{1}{2} s$, the half-side of the dodecahedron.

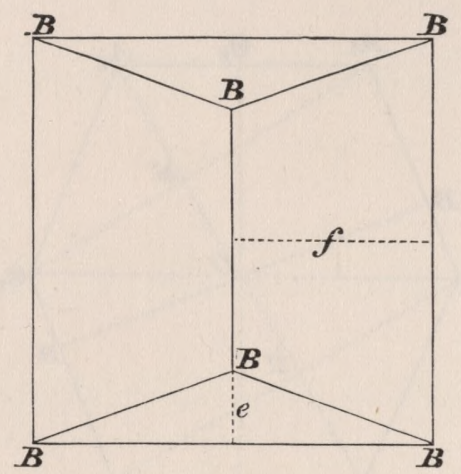

We have in this result the most simple means of forming the perspective delineation of the dodecahedron.

133. For the icosahedron the section through two opposite edges is a hexagon, as shown in the figure (p. 216): to construct it, we take the four distances $O \Theta$ each $=\rho=s \cdot \frac{1}{4}(1+\sqrt{ } 5)$ meeting at right angles; and then the distances $A \Theta_{2}, A \Theta_{4}$ each $=\frac{1}{2} s$; and complete the hexagon. This gives the sides $A \Theta_{1}, A \Theta_{3}$ each $=s \cdot \frac{1}{2} \sqrt{ } 3$, the altitude of the triangular face, side $=s$; and then, taking $\Theta_{1} B$ one-third of this, $=s \frac{1}{2 \sqrt{ } 3}$, we have $O B$ at right angles to $A \Theta_{1}$, and $O A, O B, O \Theta=R, r, \rho$ respectively.

Moreover, joining $A_{1} \Theta_{2}$ and $O A_{2}$, we have these lines cutting at right angles in a point $M$ : we find

$$
\begin{aligned}
& A_{1} \Theta_{3}=s \cdot \frac{1}{2} \sqrt{ }(5+2 \sqrt{ } 5) \\
& M \Theta_{3}=s \sqrt{\frac{5+2 \sqrt{ } 5}{20}} \\
& A_{1} M=s \sqrt{\frac{5+\sqrt{ } 5}{10}} \\
& O M=s \sqrt{\frac{5+\sqrt{ } 5}{40}},=\frac{1}{2} A_{1} M, \\
& A_{2} M=s \sqrt{\frac{5-\sqrt{ } 5}{10} .}
\end{aligned}
$$

134. It may be remarked that $A_{1} \Theta_{3}, A_{3} \Theta_{1}$ are the projections of two pentagons in planes perpendicular to that of the paper, their centres being $M, M$ : producing $O M, O M$ to the points $A_{2}, A_{4}$ respectively, we have a pentagonal pyramid, summit $A_{2}$, standing on the first pentagon, and an opposite pyramid, summit $A_{4}$, standing on 
the other pentagon: the $5+5$ triangular faces of the two pyramids are ten of the faces of the icosahedron, and the remaining ten faces are the triangles each having for its base a side of the one pentagon, and for its vertex a summit of the other

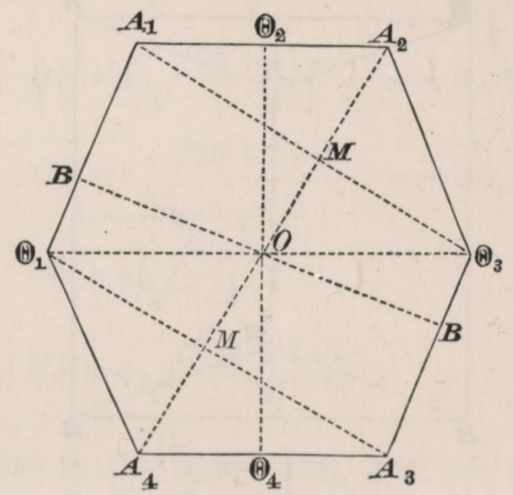

pentagon, viz. the sides are the sides of the skew decagon obtained by joining in order the angular points of the two pentagons. We have thus a convenient method of forming the perspective delineation of the icosahedron. 